University of Louisville

ThinkIR: The University of Louisville's Institutional Repository

Electronic Theses and Dissertations

$12-2011$

\title{
Optimal charging scheduling for battery electric vehicles under smart grid.
}

Nur Dayana Abd Rahman

University of Louisville

Follow this and additional works at: https://ir.library.louisville.edu/etd

Part of the Industrial Engineering Commons

\section{Recommended Citation}

Abd Rahman, Nur Dayana, "Optimal charging scheduling for battery electric vehicles under smart grid." (2011). Electronic Theses and Dissertations. Paper 1.

https://doi.org/10.18297/etd/1

This Master's Thesis is brought to you for free and open access by ThinkIR: The University of Louisville's Institutional Repository. It has been accepted for inclusion in Electronic Theses and Dissertations by an authorized administrator of ThinkIR: The University of Louisville's Institutional Repository. This title appears here courtesy of the author, who has retained all other copyrights. For more information, please contact thinkir@louisville.edu. 


\title{
OPTIMAL CHARGING SCHEDULING FOR BATTERY ELECTRIC VEHICLES
} UNDER SMART GRID

\section{By}

Nur Dayana Abd Rahman

B.E., Vanderbilt University, 2009

\author{
A Thesis \\ Submitted to the Faculty of the \\ University of Louisville \\ J.B. Speed School of Engineering \\ as Partial Fulfillment of the Requirements \\ for the Professional Degree \\ Master of Science \\ Department of Industrial Engineering \\ University of Louisville \\ Louisville, Kentucky
}

December 2011 


\section{OPTIMAL CHARGING SCHEDULING FOR BATTERY ELECTRIC VEHICLES UNDER SMART GRID}

Submitted by:

Nur Dayant/Abd Rahman
A Thesis Approved on
$1 i / 28 / 2011$
(Date)

by the following Reading and Examination Committee:

Dr. Lihui Bai, Thesis Director

Dr. Gerald W. Evans

Đr. Michael L. McIntyre 


\section{ACKNOWLEDGEMENTS}

I would like to express my deepest gratitude to my thesis advisor, Dr. Lihui Bai, for her never ending support and motivation. Her guidance along the way was really valuable and her patience and encouragements never failed to make me feel confident again in the research that I am doing. My sincere thanks go to Dr. Gerald Evans and Dr. Michael McIntyre for reviewing and providing some comments to improve this thesis. My appreciation also goes to Dr. Matthew Turner for his valuable insights on the technical aspects of this research.

I would also like to thank my sponsor, the Public Service Department of Malaysia for providing me the financial support and giving me the opportunity to pursue my postgraduate studies here at University of Louisville.

Special thanks to my dear husband, Ahmad Zharif Md Azmi, for his unwavering love and motivation for me throughout my hard times in the journey to complete this

thesis. My greatest appreciation goes to my beloved parents, Abd Rahman Md Sa'ed and Noriah Hamit for their love and support. Without their trust, I would have never reached this far.

Last but not least, I would like to thank my professors and fellow friends for their support, helpful comments and encouragement. Their help is deeply appreciated. 


\title{
ABSTRACT \\ OPTIMAL CHARGING SCHEDULING FOR BATTERY ELECTRIC VEHICLES UNDER SMART GRID
}

\author{
Nur Dayana Abd Rahman
}

November 28, 2011

A projected high penetration of battery electric vehicles (BEVs) in the market will introduce an additional load in the electricity grid. Furthermore, uncontrolled BEV charging from residential users will exacerbate the existing peak load during evening hours. In this thesis, we propose two optimization models to alleviate the impact of extra demand from electric vehicles on the power grid. The first is a centralized charging scheduling model that coordinates the charging among BEV users under the goal of minimizing the total electricity cost for all users. The second model uses a decentralized agent-based approach to scheduling the BEV charging. This approach allows each user to minimize his/her own electricity cost through a learning process on a day-to-day basis. Our numerical results indicate that the centralized model is effective in reducing the total cost and peak-to-average ratios of the system load. Although the decentralized model is less effective compared to the centralized model, it is more appealing to public. 


\section{TABLE OF CONTENTS}

ACKNOWLEDGEMENTS ...................................................................................... ii

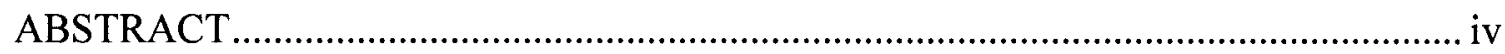

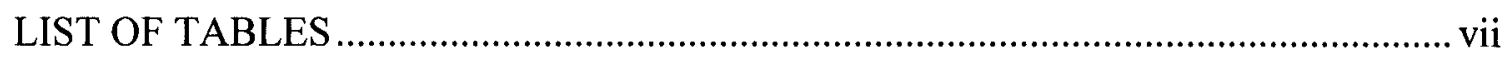

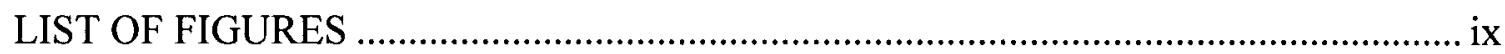

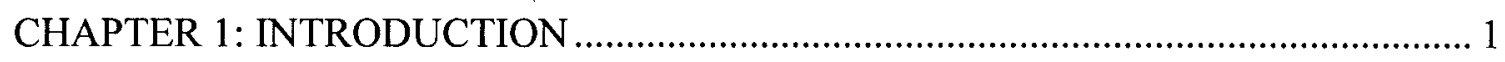

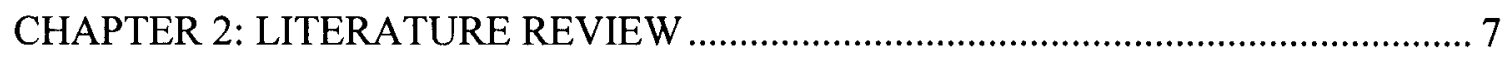

CHAPTER 3: CENTRALIZED OPTIMIZATION MODELS........................................... 14

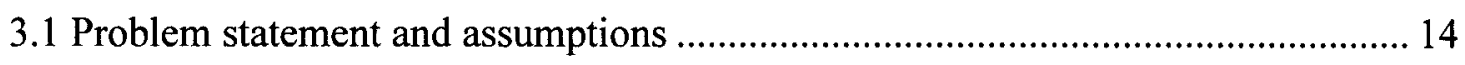

3.2 Generating baseline demand from household usage ........................................... 16

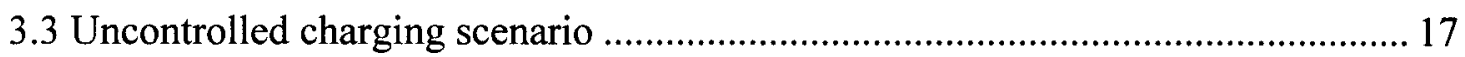

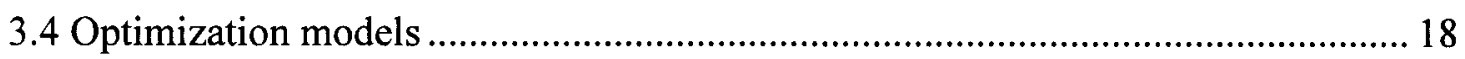

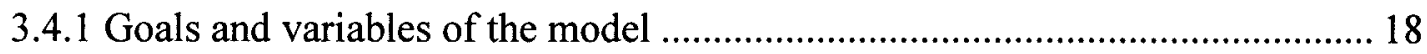

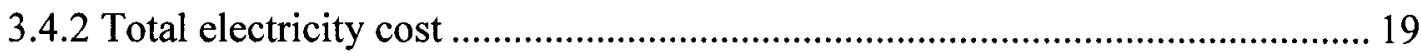

3.4.3 Optimal charging with night charging only ................................................... 20

3.4.4 Optimal charging with day-and-night charging................................................ 21

3.5 Time-of-use pricing structure ............................................................................ 24

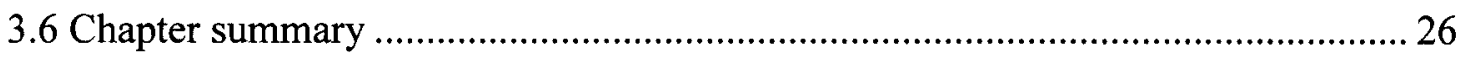

CHAPTER 4: DECENTRALIZED OPTIMIZATION MODEL ………………............... 27

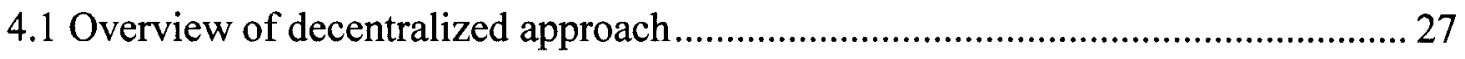

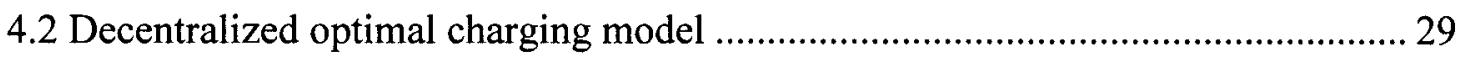

4.3 Coordination mechanisms among agents.......................................................... 31

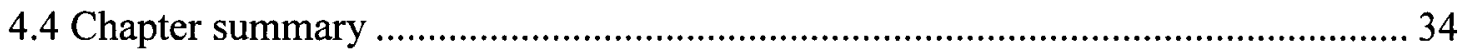

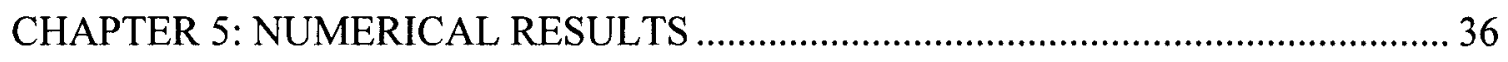

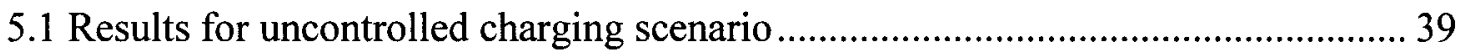

5.1.1 Results for uncontrolled charging under linear cost function............................ 39 
5.1.2 Results for uncontrolled charging under time-of-use (TOU) cost.................. 42

5.2 Results for centralized optimal charging models .............................................. 44

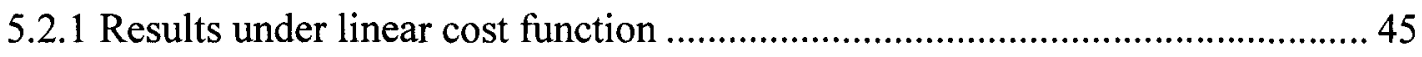

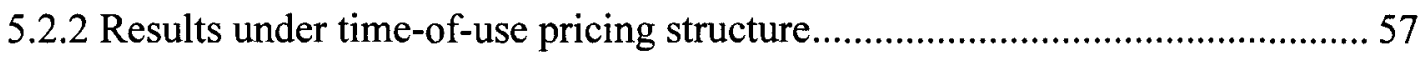

5.3 Results for decentralized optimal charging model ......................................... 66

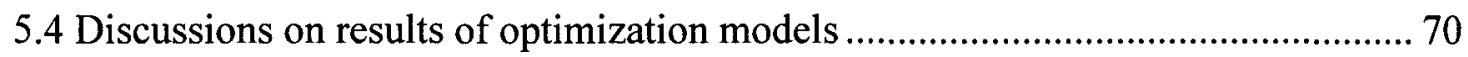

CHAPTER 6: CONCLUSIONS AND FUTURE WORK.......................................... 72

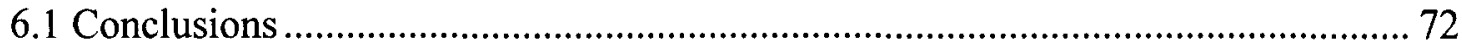

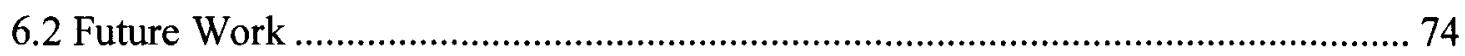

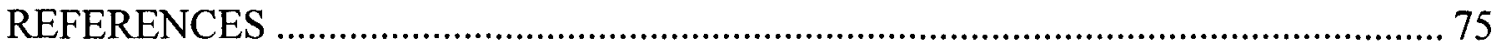

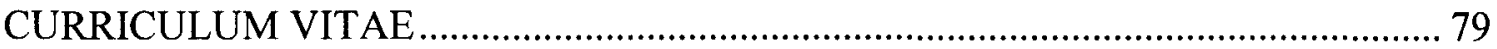




\section{LIST OF TABLES}

Table 1: System parameters in the optimization models ................................................. 19

Table 2: Time-of-use pricing structure from Collins and Mader (1983) .......................... 25

Table 3: Problem sizes for centralized models (linear cost) …………………………... 37

Table 4: Problem sizes for centralized models (TOU) ………………………………..... 38

Table 5: Problem sizes for decentralized model ............................................................... 39

Table 6: Results for uncontrolled charging under linear cost (10 users) ...........................40

Table 7: Results for uncontrolled charging under linear cost (50 users) .......................... 41

Table 8: Results for uncontrolled charging under linear cost (100 users) ......................... 41

Table 9: Results for uncontrolled charging under linear cost (200 users) ......................... 41

Table 10: Results for uncontrolled charging under TOU (10 users) ................................. 43

Table 11: Results for uncontrolled charging under TOU (50 users) ................................. 43

Table 12: Results for uncontrolled charging under TOU (100 users) ............................... 44

Table 13: Results for uncontrolled charging under TOU (200 users) ............................... 44

Table 14: Results for night charging model under linear cost (10 users) .......................... 45

Table 15: Results for night charging model under linear cost (50 users) ......................... 46

Table 16: Results for night charging model under linear cost (100 users) ........................46

Table 17: Results for night charging model under linear cost (200 users) ........................ 47

Table 18: Optimal night charging versus uncontrolled charging (linear cost) ................. 48

Table 19: Results for day-and-night charging model under linear cost (10 users) ........... 51

Table 20: Results for day-and-night charging model under linear cost (50 users) ........... 52

Table 21: Results for day-and-night charging model under linear cost (100 users) ......... 52

Table 22: Results for day-and-night charging model under linear cost (200 users) ......... 52

Table 23: Optimal day-and-night versus uncontrolled charging (linear cost) ................... 53

Table 24: Results for night-only charging under TOU (10 users) .................................... 58

Table 25: Results for night-only charging under TOU (50 users) .................................... 58

Table 26: Results for night-only charging under TOU (100 users) ..................................58 
Table 27: Results for night-only charging under TOU (200 users) ............................... 59

Table 28: Optimal night-only model versus uncontrolled charging under TOU.............. 59

Table 29: Results for day-and-night charging under TOU (10 users) ............................ 61

Table 30: Results for day-and-night charging under TOU (50 users) ............................61 61

Table 31: Results for day-and-night charging under TOU (100 users) ........................... 62

Table 32: Results for day-and-night charging under TOU (200 users) ............................ 62

Table 33: Optimal day-and-night charging versus uncontrolled charging under TOU .... 62

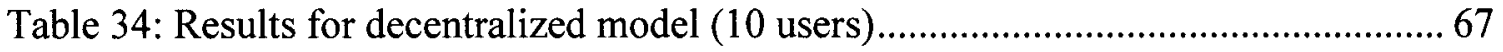

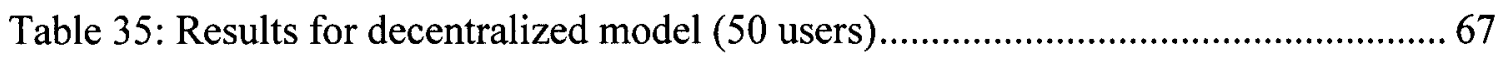

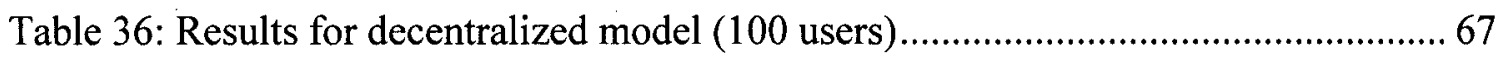

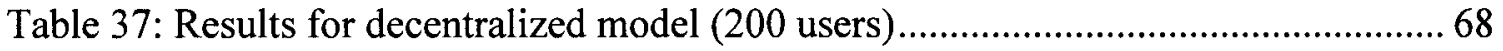

Table 38: Summary of results for decentralized model .............................................6 68

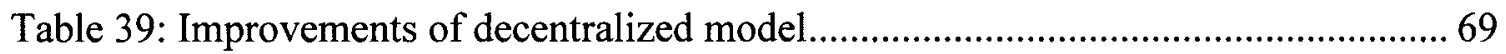




\section{LIST OF FIGURES}

Figure 1: Projected liquid consumption by end-use sector ................................................. 2

Figure 2: The household load profiles in a typical summer month ................................. 17

Figure 3: Algorithm of the decentralized model.......................................................... 32

Figure 4: Load profile with uncontrolled EV charging under linear cost.......................... 42

Figure 5: Load leveling under optimal night charging model .......................................... 49

Figure 6: Uncontrolled charging profile for User 1 .................................................. 50

Figure 7: Optimal nighttime charging schedule for User 1 .........................................5

Figure 8: Load leveling under optimal day-and-night charging model ............................ 54

Figure 9: Optimal day-and-night charging schedule for User 1 ..................................... 55

Figure 10: Overall load leveling under linear cost function ............................................56

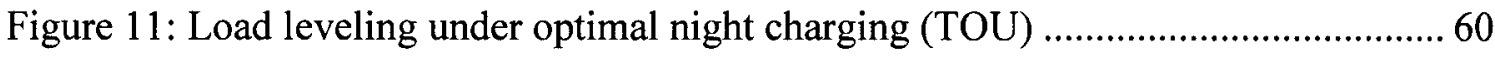

Figure 12: Load leveling under optimal day-and-night charging (TOU) .......................... 63

Figure 13: Charging profile for User 1 under time-of-use pricing structure ..................... 64

Figure 14: Overall load leveling under TOU pricing structure............................................65

Figure 15: Residential load profile under decentralized model ........................................... 69 


\section{CHAPTER 1: INTRODUCTION}

The depletion of non-renewable energy resources such as fossil fuels has been a growing concern worldwide. For example, Shafiee and Topal (2009) predicted that the oil and gas reserves will only last until 2042 while coal is expected to be available until 2112. Furthermore, fossil fuels such as coal, natural gas and oil are among the primary sources to generate energy for electricity and transportation. On the other hand, according to a report by Energy Information Administration (2011), the world energy consumption is anticipated to grow up to 53 percent from 2008 to 2035 , and similar increase of energy demand for the transportation section is anticipated as well. The latter is further supported by the conclusion from The Energy Foundation (2006) that transportation is accountable for two-thirds of United States oil consumption.

Particularly, Energy Information Administration (2011) projects the demand for liquid fuels in transportation sector to increase over the next 25 years as shown in Figure 1. Given the world's increasing dependency on energy and the rapid declining in the abundance of non-renewable fossil fuels, the development of renewable energy related technologies, such as solar energy, wind power and alternative-energy vehicles, has been the focus for many research disciplines. 


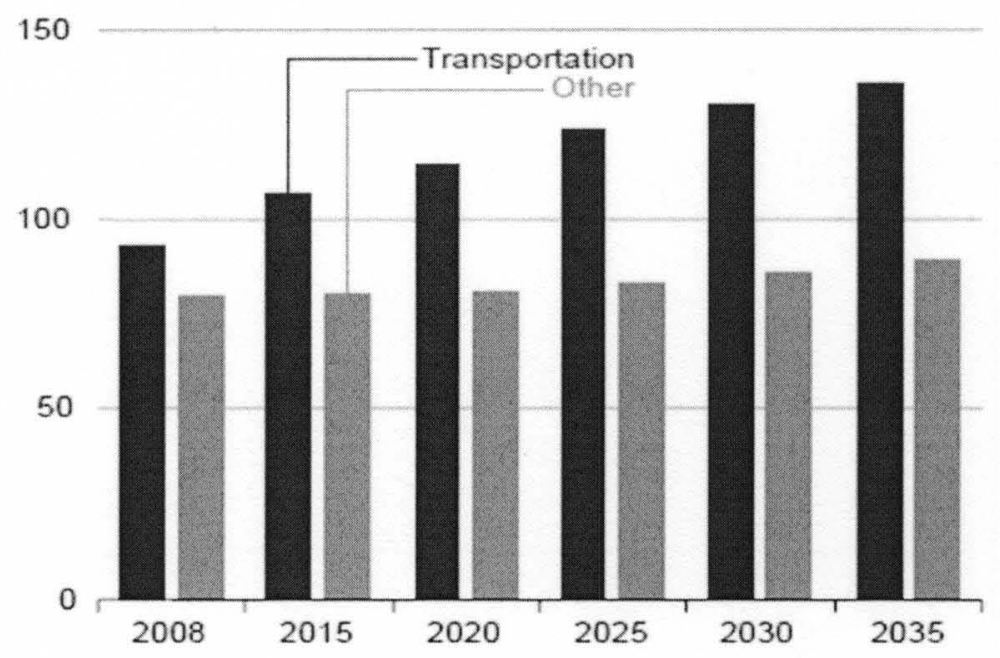

Figure 1: Projected liquid consumption by end-use sector

(Source: Energy Information Administration, 2011)

Apart from the declining rate of fossil fuel reserves, the burning of fossil fuels for energy generation poses a detrimental effect on the environment. Carbon dioxide, which is a by-product of burning fossil fuels, is one of the main factors of global warming. Furthermore, an annual report (The Energy Foundation, 2006) found transportation to be accountable for more than 25 percent of total United States global warming pollution. The same report also concluded that light duty vehicles (LDVs) contribute to the 60 percent of global warming pollution caused by transportation sector in the United States. It has also been reported that automobiles emit about ten percent of global carbon dioxide emissions from fossil fuels (see, e.g., DeCicco, Fung \& An, 2006).

Recognizing that automobiles, particularly LDVs, are largely responsible for the increasing amount of energy consumption, and thus greenhouse gas emissions, numerous studies have focused on developing alternative vehicles that would help curb the detrimental effects of greenhouse gas emissions on the environment. In addition, the 
development of alternative vehicles is also essential in decreasing the energy dependence on non-renewable energy resources.

One potential solution to this problem is through the introduction of electric vehicles such as battery electric vehicles (BEVs) and plug-in hybrid electric vehicles (PHEVs). These electric vehicles are mainly enabled by high-efficiency electric motor and controller, and powered by alternative energy sources. Hence, electric vehicles could provide the means for a cleaner, more efficient and environmentally friendly urban transportation system. In addition, Ipakchi and Albuyeh (2009) conclude that electric car users would also experience reduced operational costs when compared with cars that run on gasoline.

However, with a projected high penetration of electric vehicles in the market along with the recent goal by President Obama to put one million electric vehicles on the road by 2015 , there have been growing concerns on the potential impacts of electric vehicles on the current electricity grid. Ipakchi and Albuyeh (2009) noted that BEVs will represent a considerable new load on the existing primary and secondary distribution networks. Moreover, since PHEVs need 0.2-0.3 kWh of charging power for one mile of driving (Mohsenian-Rad, Wong, Jatskevich \& Schober, 2010), a substantial new load may be present in the existing distribution system particularly doubling the average household load during the charging time which often coincides with the peak load hours during the evening.

Furthermore, the additional load contributed to charging electric vehicles could potentially lead to an increase in our electric bills. The increase in cost is mainly 
associated with the method of electricity generation utilized by the power plants. Currently, there are three basic categories of power plants in the United States which are fossil fuel, nuclear and hydropower. Fossil fuel, such as coal, is usually used as a primary source of electricity generation by power companies since it is considered as one of the most inexpensive energy sources. Hence, fossil fuel is mainly used to generate electricity during base periods. However, during peak hours, power companies rely on more expensive generators such as natural gas, diesel or hydropower to meet the extra demands from customers. As a result, the cost of electricity during peak hours increases.

Due to these issues, many studies have aimed to develop mechanisms to mitigate the impacts of charging electric vehicles on the electricity grid. For example, demand response (DR) programs intend to manage customer's electricity consumption in response to supply conditions by encouraging electricity users to reduce their energy demand, thus reducing the peak demand for electricity. The second mechanism that has been the focus of many studies is the demand side management (DSM).

In the context of alleviating potential increase of peak load due to high penetration of electric vehicles, DSM technologies adopt smart control mechanisms to encourage end users to be more efficient in consuming energy using price as an incentive. For example, users would shift their energy consumption such as using appliances and charging electric vehicles to off-peak hours where the price is much cheaper. Furthermore, managing the use of electricity during peak hours could help maintain a lower electricity cost since the utility companies would depend less on the expensive sources of electricity generation. 
According to Geillings (1985), some of the typical forms of load shaping that have been implemented are such as peak clipping, valley filling and load shifting. These objectives are widely adapted in alleviating the impact of electric vehicles' charging on the electrical distribution system. Moreover, the initiatives of DSM technologies such as a smart meter are envisioned to become more possible in the advent of Smart Grid. Fan (2010) defines Smart Grid as "an intelligent electricity network that integrates the actions of all users connected to it and makes use of advanced information, control, and communication technologies to save energy, reduce cost and increase reliability and transparency."

This thesis, which lies in the stream of DSM, aims to reduce the effects of uncontrolled residential charging by BEV users. Different models are developed to "smartly" schedule charging by the BEV users such that the overall load is leveled and cost is minimized. The first set of models study the centralized scenario which assumes that all users cooperate with the decision of a central controller on when to charge their electric vehicles. Several different models are proposed to study the night-charging-only and day-and-night-charging scenarios, with considerations of various cost structures for unit electricity price. The unit electricity cost functions that we study include a linear increasing function of the load at any time interval $t$ and a time-of-use function. The second set of models considered in this research is the decentralized model. The decentralized model assumes that users do not follow the schedule by central controller; rather, they optimize their own charging schedule to minimize their own electricity cost. They also learn and adapt to the "near-optimal" charging schedule on a day-to-day basis. 
Results from these two models are then compared to conclude on the best approach to schedule the BEV charging.

The rest of the thesis is organized as follows. Chapter 2 reviews the literature of DSM under Smart Grid. Chapter 3 formulates a centralized model for optimal charging scheduling of electric vehicles in residential areas. Chapter 4 presents the decentralized model for optimal charging scheduling of electric vehicles in residential areas. Chapter 5 discusses the numerical results of models in Chapters 3 and 4. Finally, Chapter 6 summarizes the thesis and discusses possible future work. 


\section{CHAPTER 2: LITERATURE REVIEW}

In this thesis, we focus on developing an optimal charging schedule for electric vehicle users in residential areas. Several studies have showed that uncoordinated PHEV charging at high penetration rates could increase system peak load, losses and decreases system load factor (see, e.g., Taylor et al., 2009; Clement-Nyns, Haesen \& Driesen, 2010). Furthermore, Rahman and Shrestha (1993) noted that the impact of electric vehicle charging depends on when it is being charged and the charging pattern. Rahman and Shrestha (1993) indicated that a substantial new peak might be present in the early off-peak period when a considerable amount of electric vehicles start to charge. Hence, there exists a need to encourage a more distributed charging profile among electric vehicle users.

A potential solution to mitigate the impact of uncontrolled charging by electric vehicle users on the electricity grid is through coordinated charging. The implementation of coordinated charging is envisaged to become more promising with the development of smart grid communication infrastructure. Clement-Nyns, Haesen and Driesen (2010) proposed a centralized optimal charging in their paper to minimize the power losses on the residential grid. A real-time coordination of multiple PHEVs on the distribution network which minimizes the total electricity generation cost and the corresponding energy losses has been developed to address this issue by Deilami et al. (2011). 
Similarly, Sortomme, Hindi, MacPherson and Venkata (2011) developed coordinated charging algorithms to reduce the effects of uncontrolled charging on system losses. In their work, they presented three algorithms: minimization of loss, load variance minimization and load factor maximization. The convex quadratic program presented in their paper solves for the optimal charging profile of each PHEV user in the system. Sortomme et al. (2011) showed that coordinated charging outweighs uncontrolled charging at different levels of PHEV penetrations (e.g., 10\%, 20\%, 50\% and 100\%).

Numerous optimization techniques have been used in accomplishing the scheduling models. Some examples are such as mixed integer linear programming (see, e.g., Zhang et al., 2011), quadratic programming (see, e.g., Vandael et al., 2010; Clement-Nyns, Haesen \& Driesen, 2010; Ramchurn et al., 2011) and dynamic programming (see, e.g., Kowahl \& Kuh, 2010). One of the objectives of the optimization is to schedule users' energy usage while reducing their total cost of electricity. Obara (2007) developed a computer program that optimizes the arrangement of equipment in each building that is connected to a fuel cell network, and the path of the hot water piping network to each house under the goal of cost minimization. Mohsenian-Rad et al. (2010) presented a convex cost minimization model for residential users under the deployment of energy consumption scheduling (ECS) devices.

Moreover, electricity price, in particular, has been viewed as one of the incentives for users to shift their electricity usage to off-peak hours. In the energy consumption model proposed by Mohsenian-Rad et al. (2010), they showed that pricing and billing are essential in encouraging them to use the proposed ECS. The electricity price considered by Mohsenian-Rad et al. (2010) includes quadratic cost function. They conclude that the 
models they developed decrease the peak-to-average ratio (PAR) and the total energy cost by a substantial amount, $38.1 \%$ and $37.8 \%$ respectively. In a related study, Vytelingum et al. (2010) incorporated market prices which are a monotonically and rapidly increasing function of supply in their optimization model to compute the best energy storage profile for each user in the system. They showed that minimizing total energy cost is important in regulating user's behavior.

Fan (2010), on the other hand, studied the application of congestion pricing in Internet traffic control for demand response in smart grid. Fan (2010) discussed in his paper that pricing is an important factor in regulating user's demand. In his paper, he proposed the concept of "willingness to pay" which was adapted from the work of Kelly, Maulloo and Tan (1998). The results from his simulations showed that "willingness to pay" parameter has a profound effect on how the user regulates his or her demand. Moreover, the parameter also indicates how the user is reacting to the price signals to maximize his or her own benefits. Fan (2010) concludes that the overall load can be leveled via the congestion pricing information.

Most studies on electricity markets incorporate quadratic functions in establishing the relationship between cost and electric usage (see, e.g., Martinez-Budria, Jara-Diaz \& Ramos Real, 2003; Fetz \& Filippini, 2010; Mohsenian-Rad et al., 2010) with the simplest form of the quadratic function applied as the Taylor expansion of order two. Moreover, quadratic forms are typically used in power systems research to represent total costs of operations (see, e.g., Shahidehpour, Yamin \& Li, 2002). In the latter, a piecewise linear approximation is often applied to ease computational burden that would otherwise be experienced by quadratic models. 
Particularly, Martinez-Budria et al. (2003) implemented the quadratic cost function to increase the productivity of the electric sector in Spain for the years of 19851996. Mohsenian-Rad et al. (2010), on the other hand, applied a convex cost function in the form of a smooth and differentiable quadratic function to represent the actual cost of energy being used. In a related study by Cain and Alvarado (2004), they showed a comparison between the uses of piecewise cost compared to quadratic cost on the bid format of electricity market. They conclude that quadratic function facilitates analysis while piecewise linear function may incur some complexity in the analysis.

In this thesis, we also consider the impact of time-of-use (TOU) rates on electric vehicle users' energy consumption as a part of DSM. Over the past decades, the Department of Energy has been actively engaged in developing residential TOU rates which varies according to the time-of-day and season. The first project to implement the residential TOU rate began in 1975 in Vermont (see, e.g., Aigner, 1985). Aigner (1985) provided a detailed analysis of the TOU experiments in residential areas conducted by Department of Energy with the primary focus on price elasticity. The results from his paper initiated the basis of whether the public utilities and the regulatory commissions should consider voluntary or mandatory TOU pricing structure on residential areas. Aigner (1985) concludes that the experiments helped focus on some important issues for policy makers such as effective pricing mechanism and consumer behavior.

Correspondingly, many studies have also aimed to analyze the impacts of TOU rates on residential areas (see, e.g., Hartway, Price \& Woo, 1999; Baladi, Herriges \& Sweeney, 1998; Collins and Mader, 1983). In particular, the findings from Collins and Mader (1983) motivated our study to analyze the effects of TOU pricing structure on 
determining the optimal charging schedule for electric vehicle users. We choose to use the TOU rate proposed in Collins and Mader (1983) because they introduce several prices during the off-peak hours to prevent simultaneous start of charging from all users.

Another factor that we considered in the scheduling problem in this thesis is the electric vehicle charging pattern. The charging pattern of an electric vehicle is particularly dependent on the vehicle's usage such as the distance travelled and when the recharging is needed. Collins and Mader (1983) examined the different timings of electric vehicle charging with the assumption that most of the charging occurs at home. Further, they stated in their work that the EVs will not actually travel for its full range each day. Additionally, they noted that some EV drivers will consider a "safety margin" to ensure an adequate amount of charge to return home.

Koyanagi and Uriu (1998) addressed the concern on charging durations of vehicles with different travel motions. They proposed a regional charging shift model for nighttime recharging to mitigate the problem of simultaneous charging from the electric vehicle users. Using real data of vehicles' performance (in $\mathrm{km}$ ) and their energy consumption (in Wh/km) in Tokyo, Koyanagi and Uriu (1998) conclude that the demand from business cars have a more profound impact on the electricity grid with a demand of 14.6 GW compared to personal cars $(7.6 \mathrm{GW})$ based on a daily forecast. The significant difference in the demand is attributed to the cruising radius of the vehicles. They also noted that electric vehicles with long cruising radius, for instance bus and taxi, would need several quick recharging during the day. 
Finally, this thesis also studies a decentralized optimal scheduling method, and proposes an agent-based approach to scheduling EV chargings for all users. In an agentbased modeling, Bonabeau (2002) puts, "the system is modeled as a collection of autonomous decision-making entities called agents." Each agent is capable of evaluating its own situation and makes decisions based on some conditions. According to Bonabeau (2002), there are various advantages for the agent-based approach in modeling such as encapsulating any surfacing event, describing the nature of a system and providing flexibility in responding to dynamic conditions. Hence, the agent-based approach would be more beneficial in modeling an optimal scheduling model where each electric vehicle user is allowed to decide on his or her local charging schedule.

Recently, many have studied decentralized control systems in demand side management (DSM) with the agent-based approach. For example, Vytelingum et al. (2010) implemented the agent-based concept in developing a micro-storage management algorithm for the smart grid. Vytelingum et al. (2010) proposed a strategy to predict the optimal storage profile based on a day-ahead strategy. Under this strategy, each agent fixes his or her storage profile based on the following day's forecasted market price. They also found that the average storage profile converged to Nash Equilibrium of the system; each user charges during off-peak hours and discharges during peak hours.

Furthermore, Vandael et al. (2010) used a decentralized model to level the load at each transformer through two coordination strategies: the energy limiter and power limiter. The energy limiter uses forecasts loads while the power limiter eliminates the need for any data predictions. They conclude that the decentralized model provide more adaptability in determining each PHEV user's charging schedule. Finally, Galus and 
Andersson (2008) developed a model to control PHEV charging behavior by incorporating individual preference curves to attain optimal charging in distribution networks. Galus and Andersson (2008) showed that personal preference and electricity price affect how the PHEV users schedule their charging profile.

In Chapters 3 and 4, we will define the charging scheduling problem for BEV users in residential areas. We will develop the formulation for the centralized and decentralized models and explain the variables that are considered in the respective models. 


\section{CHAPTER 3: CENTRALIZED OPTIMIZATION MODELS}

\subsection{Problem statement and assumptions}

This chapter focuses on modeling the centralized optimization models to schedule electric vehicle users' charging activities. The main objectives are as follows:

1. Minimize the total electricity cost incurred to all users.

2. Determine the amount and time to charge for each user while still meeting the energy demands from both household usage and electric vehicles.

Several major assumptions are made in developing the centralized optimization models. First, we envision that devices such as smart meters will be available in the advent of Smart Grid. These devices are essential for users to adjust their electricity consumption based on the decision of a central controller. Moreover, the optimization models assume that all users will cooperate with the scheduling decision by the central controller.

Second, without loss of generality we only include BEV in our models. If other electric vehicle users such as PHEV users need to be considered, simply changing the charging demands will accommodate the inclusion of PHEV users. For pure battery charged electric vehicles, the Nissan LEAF is chosen in this study. The Nissan LEAF is a $100 \%$ electric vehicle which comprises of a $24 \mathrm{kWh}$ lithium-ion battery (Nissan LEAF, 
n.d.). However, the battery is only limited to a Depth of Discharge (DOD) of $80 \%$. Hence, the battery is estimated to complete its charging in approximately 5.8 hours. We also assume that this charging duration is achieved under a Level 2 charger $(240 \mathrm{~V})$ which is available to BEV users in residential areas.

Travel motions of the BEV users are also taken into account in the optimization models. This factor is incorporated due to the different charging durations needed for different amount of distance travelled as proposed by Koyanagi and Uriu (1998). The two types of users considered in this study are:

1. Short-distance users: Travel less than 100 miles (roundtrip) daily and require only $24 \mathrm{kWh}$ of battery capacity.

2. Medium-distance users: Travel between 100-150 miles (roundtrip) daily and require approximately $36 \mathrm{kWh}$ of battery capacity.

The distance of 100 miles is particularly chosen since it is found to be a sufficient mileage for more than $90 \%$ of all household vehicle trips in the United States (U.S. Department of Energy, n.d.; Collins \& Mader, 1983). Furthermore, the particular range of 100 miles is adequate in reducing BEV users' "range anxiety". Thus, we classify the drivers who travel less than 100 miles daily as short-distance users. The medium-distance users, on the other hand, are considered for users who travel slightly more than the projected average range. Hence, we assume this type of users will need a bit more charge to meet their travel demand. Additionally, we presume that users who travel for longer than 150 miles daily will acquire a hybrid electric vehicle instead of a pure electric vehicle due to the inconvenience of too long of a charging duration. 
We also assume that charging infrastructure at the workplace or commercial charging stations close to workplace is available to users. Hence, users are able to charge their electric vehicles when they are at work during the day. Finally, we assume that the maximum energy that may be consumed by the BEV in one hour is only up to $3.3 \mathrm{kWh}$ (Ipakchi and Albuyeh, 2009; Nissan LEAF, n.d.). As a result, the short-distance users

will need approximately 6 hours for a complete charging while the medium-distance users will need approximately 9 hours in fulfilling the charging demand. In addition, we assume $100 \%$ commitment from the users once they are given a time slot to charge their electric vehicle.

\subsection{Generating baseline demand from household usage}

In this thesis, we focus on a summer load profile for $n$ residential users which is usually defined as the months of June, July, August and September. The summer load profile, which contains a single peak, is chosen for this analysis due to an increased demand for electricity attributed to the hot weather. From a report by NAHB Research Center (2001) at Southern California Edison (SCE) territory, three categories of household load profiles are identified and they are described as follows:

1. Low-usage load profile: $23-28 \mathrm{kWh}$ daily

2. Average-usage load profile: $29-36 \mathrm{kWh}$ daily

3. High-usage load profile: $51-62 \mathrm{kWh}$ daily

Using the above information, we randomly generated a baseline household demand profile for half-hour duration throughout a 24-hour interval for the three types of users. 
The randomly created "household-only" demand profiles are illustrated in Figure 2. In this figure, maximum, average and minimum represents daily usage of 51-62 kWh, 29-36 $\mathrm{kWh}$ and 23-28 kWh respectively. These profiles exhibit the same peak and off-peak loads and periods as shown in the empirical data in SCE territory (NAHB Research Center, 2001).

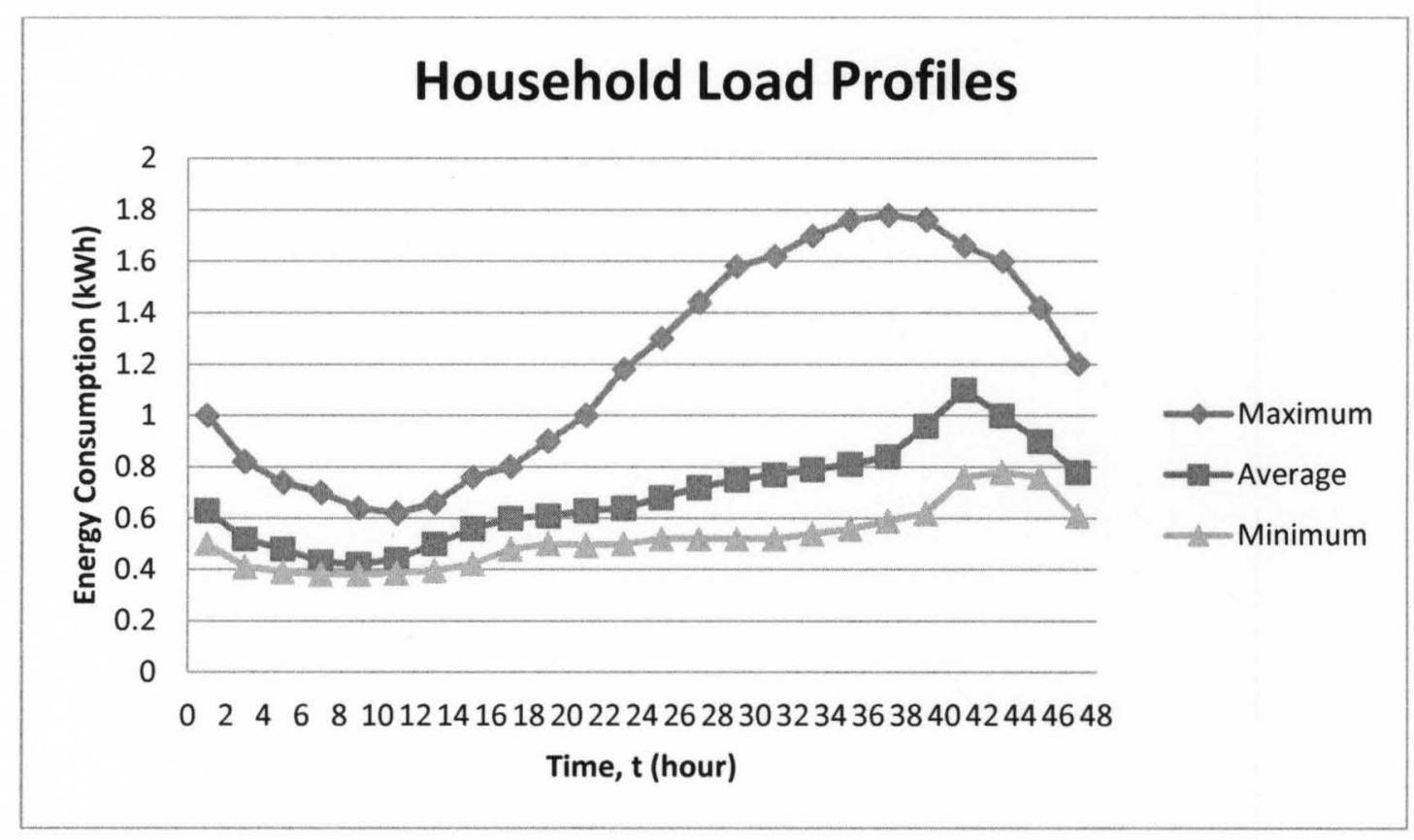

Figure 2: The household load profiles in a typical summer month

\subsection{Uncontrolled charging scenario}

This section studies the impact of uncontrolled EV charging on the household load profiles. We assume, when left uncontrolled, EV users generally choose the most convenient time during the evening hours between $6: 00 \mathrm{pm}$ to 7:00 am the following day, to charge their EVs. We randomly generate starting times for the medium-distance and short-distance users to charge their vehicles. Several assumptions are made in this data 
generation. First, as mentioned previously, we assume that the medium-distance and short-distance users will charge between 6:00 pm to 7:00 am daily. Second, a total charge of $1.65 \mathrm{kWh}$ (for half an hour) is assigned if the BEV user charges in time interval $t$. Finally, we also assume that once charging starts, it is done continuously until the energy demand from each type of BEV user is fulfilled.

\subsection{Optimization models}

\subsubsection{Goals and variables of the model}

We consider a set of $n$ residential users comprised of three categories of household load profiles as described in Section 3.2. The corresponding household demand from each user $i$ at time interval $t$ is defined as $D_{i}^{t}$. Here, we define a 24-hour cycle that consists of 48 half-hour intervals which starts at 12:00 am until 11:30 pm. Further, we assume that the set of $n$ residential users include short-distance and mediumdistance BEV users as explained in Section 3.1. For each BEV user $i$, we define the charging requirements/demands as $d_{i}$.

The centralized optimal charging scheduling model aims to schedule a charging profile for each user $i$ at time interval $t$, represented by variable $x_{i}^{t}$ such that the total charge assigned fulfills the user's charging needs and the total electricity costs for all users collectively are minimized. As discussed in Section 3.3, a charge of $1.65 \mathrm{kWh}$ is allocated to each user $i$ at the corresponding time interval $t$. In our model, the total load $v_{t}$ at each time interval $t$ is calculated as the sum of household load and extra demand 
from BEV of all users during that particular time interval. The mathematical representation of the total load at time interval $t$ is given in Equation 3-1:

$$
v_{t}=\sum_{i=1}^{n}\left(D_{i}^{t}+x_{i}^{t}\right)
$$

\subsubsection{Total electricity cost}

We adopted the linear function, as proposed by Mohsenian-Rad et al. (2010), in calculating the unit electricity cost. In particular, the unit price function $p_{t}\left(v_{t}\right)$ at time interval $t$ is given in Equation 3-2.

$$
p_{t}\left(v_{t}\right)=C_{0}+C v_{t}
$$

where $C_{0}=0.071$ and $C=0.02$. Under this pricing structure, the unit price of electricity at time interval $t$ is an increasing function of the total load during that time. Such a pricing structure provides an incentive for households to use electricity during off-peak hours when electricity is cheaper. Thus, it is an effective tool for load leveling. Table 1 summarizes the system parameters considered in this study.

Table 1: System parameters in the optimization models

\begin{tabular}{|l|l|}
\hline \multicolumn{2}{|c|}{ Indices } \\
\hline Users, $i$ & $1,2, \ldots, \mathrm{n}$ \\
\hline Time, $t$ & $1,2, \ldots, 48$ \\
\hline \multicolumn{2}{|c|}{ Parameters } \\
\hline$d_{i}$ & Demand (in kWh) from EV from user $i$ \\
\hline$D_{i}^{t}$ & Household load (in kWh) of user $i$ at time $t$ \\
\hline$v_{t}$ & $\begin{array}{l}\text { Total load which is a sum of household load and extra } \\
\text { demand from BEVs (in kWh) from all users at time } t\end{array}$ \\
\hline$p_{t}\left(v_{t}\right)$ & Unit electricity price (in \$) at time $t$ \\
\hline
\end{tabular}




\subsubsection{Optimal charging with night charging only}

This section focuses on the scenario where BEV users only charge their vehicles when they are at home which is from 6:00 pm to 7:00 am. We developed a mixed integer non-linear programming model to analyze this scenario. The optimization model is as follows:

$$
\operatorname{Min} \sum_{t=1}^{48} p_{t}\left(v_{t}\right) * v_{t}
$$

Subject to:

$$
\begin{array}{cc}
v_{t}=\sum_{i=1}^{n}\left(D_{i}^{t}+x_{i}^{t}\right) & \forall t \\
\sum_{t=1}^{48} x_{i}^{t}=d_{i} & \forall i \\
\sum_{t=16}^{36} y_{i}^{t}=0 & \forall i \\
x_{i}^{t}=1.65 * y_{i}^{t} & \forall i, t \\
x_{i}^{t} \geq 0 & \forall i, t \\
y_{i}^{t} \in\{0,1\} & \forall i, t
\end{array}
$$

There are two decision variables considered in model (3-3)-(3-9). The first decision variable $x_{i}^{t}$ determines the amount of charge that BEV user $i$ will charge beginning at time interval $t$ for 30 minutes. The second decision variable $y_{i}^{t}$ is binary, where $y_{i}^{t}=1$ when BEV user $i$ charges at time interval $t$, and $y_{i}^{t}=0$ otherwise. 
The objective of model (3-3)-(3-9) is described in Equation (3-3), minimizes the total cost of electricity usage in a 24-hour duration for all users. Constraints of the model include Equation (3-4) through (3-9). Particularly, Constraint (3-4) calculates the total load in each time interval $t$ as the sum of household load and charging demand at the particular time for $n$ users. Additionally, Constraint (3-5) ensures that each BEV user's daily charging requirement is fulfilled in a 48 half-hour cycle, while Constraint (3-6) states that no user will charge their EVs during the day between 7:30 am to 5:30 pm. Further, Constraint (3-7) assigns a total charge of $1.65 \mathrm{kWh}$ to user $i\left(x_{i}^{t}=1.65\right)$ if the user is determined to charge during time interval $t\left(y_{i}^{t}=1\right)$. Since we do not consider any storage capability by the electric vehicle in this model, we only allow positive charging. Hence, Constraint (3-8) describes this constraint. Finally, Constraint (3-9) states that decision variable $y_{i}^{t}$ is a binary variable indicating if user $i$ will charge or not at time interval $t$.

\subsubsection{Optimal charging with day-and-night charging}

In this section, we discuss another scenario where BEV users are allowed to charge during the day as well as in the evening. The purpose is to investigate the benefit of "at-work-charge" to load leveling, compared to the more convenient "night-only" scenario in Section 3.4.3.

For this particular scenario, both medium- and short-distance users will be allowed to charge their BEVs twice a day: at work $(8: 00 \mathrm{am}$ to $5: 30 \mathrm{pm})$ and at home in the evening (5:30 pm to 7:30 am the following day). For medium-distance users, in order 
to ease their "range anxiety," they charge for at least an hour and at most three hours at work. This coincides with their lunch break. For short-distance users, there is no mandatory charging at work, but they are only allowed to charge no more than three hours at work.

Particularly unique to day-time charging is the constraint that BEV users will consecutively charge their cars for at least an hour, instead of 30-minute intervals, if they are assigned to charge at time interval $t$. This consideration is made because when charging at work, the sophisticated control required by a scattered 30-minute charging intervals may not be available or practical at work place or commercial charging stations close to work place. Finally as mentioned previously, the reason we allow day-time charging is to analyze if the load from electric vehicles can be shifted such that the total load is more leveled. The optimization model for this scenario is as follows:

$$
\operatorname{Min} \sum_{t=1}^{48} p_{t}\left(v_{t}\right) * v_{t}
$$

Subject to:

$$
\begin{array}{cc}
v_{t}=\sum_{i=1}^{n}\left(D_{i}^{t}+x_{i}^{t}\right) & \forall t \\
\sum_{t=1}^{48} x_{i}^{t}=d_{i} & \\
\sum_{t=17}^{36} y_{i}^{t} \geq 1 & \forall i
\end{array}
$$




$$
\begin{array}{cc}
\sum_{t=17}^{36} y_{i}^{t} \leq 3 & \multicolumn{1}{c}{\forall i} \\
x_{i}^{t}=3.3 * y_{i}^{t} & \\
& \forall i, t \\
x_{i}^{t}=1.65 * y_{i}^{t} & \in\{17, \ldots, 36\} \\
& \forall i, t \\
y_{i}^{t} \leq 1-y_{i}^{t+1} & \in\{1, \ldots, 16\} \\
& \cup\{37, \ldots, 48\} \\
x_{i}^{t} \geq 0 & \forall i, t \\
y_{i}^{t} \in\{0,1\} & \in\{17, \ldots, 36\} \\
& \forall i, t
\end{array}
$$

The optimization model for day-and-night charging is similar to the model described in Section 3.4.3. We still consider the same decision variables and objective function in this model. However, there are several new constraints needed in this model. In particular, Constraint (3-13) states that the medium-distance users are enforced to charge at least once between 8:00 am to 5:30 pm. Constraint (3-14) ensures that all users are only allowed to charge up to 3 times during the day. Constraint (3-15) represents a total charge of $3.3 \mathrm{kWh}$ which is assigned to the BEV user $i$ who is scheduled to charge between 8:00 am to $5: 30 \mathrm{pm}$. On the other hand, Constraint (3-16) a total of $1.65 \mathrm{kWh}$ is allocated to BEV user $i$ who is scheduled to charge from $5: 30 \mathrm{pm}$ to $7: 30$ am the following day. Lastly, Constraint (3-17) states that consecutive charging for two halfhour intervals is enforced to all users who are scheduled to charge during the day. 


\subsection{Time-of-use pricing structure}

In this section, we consider time-of-use (TOU) pricing structure. Under this structure, the prices of electricity usage are fixed for a specific time period. A TOU pricing structure is typically categorized to type of season such as summer or winter. Furthermore, the time periods are frequently separated into on-peak, off-peak and intermediate periods. Usually the prices to be paid for the energy consumed during these periods are already established by the electric utilities and known to users in advance. Hence, this price structure provides an incentive for users to respond to the change in price and adjust their energy consumption to a cheaper cost period. By doing so, the users will eventually reduce their overall energy bill. Currently, several utility companies have implemented the TOU pricing structure for residential users such as NV Energy in Nevada (NV Energy, n.d.), PGE in Oregon (Portland General Electric, n.d), Southern California Edison in California (Southern California Edison, n.d.), among others.

In this thesis, we implement a TOU pricing structure proposed by Collins and Mader (1983) shown in Table 2. From Table 2, several prices (in 1982 dollars) are assigned during the off-peak period to prevent simultaneous charging from all BEV users. Additionally, introducing multiple prices during off-peak period avoids a sudden spike in demand between an on-peak and off-peak periods. Equation (3-20) defines the new objective function using the TOU pricing. This should replace the objective functions (3-3) and (3-10) in the night-only scenario and day-and-night scenario, respectively. No change of constraints is needed for both scenarios under this pricing structure. 
Table 2: Time-of-use pricing structure from Collins and Mader (1983)

\begin{tabular}{|c|c|}
\hline Time of Day & Price (c/kWh) \\
\hline \multicolumn{2}{|c|}{ On-Peak } \\
\hline 7am-10pm & 8.0 \\
\hline \multicolumn{2}{|c|}{ Off-Peak } \\
\hline $10 \mathrm{pm}-11 \mathrm{pm}$ & 5.4 \\
\hline $11 \mathrm{pm}-12 \mathrm{pm}$ & 4.7 \\
\hline $12 \mathrm{pm}-1 \mathrm{am}$ & 4.5 \\
\hline 1am-2am & 4.4 \\
\hline 2am-3am & 4.3 \\
\hline 3am-4am & 4.2 \\
\hline 4am-5am & 4.5 \\
\hline 5am-6am & 4.8 \\
\hline 6am-7am & 5.0 \\
\hline
\end{tabular}

$$
\begin{aligned}
\text { Min } & \sum_{t=1}^{2} 0.045 v_{t}+\sum_{t=3}^{4} 0.044 v_{t} \\
& +\sum_{t=5}^{6} 0.043 v_{t}+\sum_{t=7}^{8} 0.042 v_{t} \\
& +\sum_{t=9}^{10} 0.045 v_{t}+\sum_{t=11}^{12} 0.048 v_{t} \\
& +\sum_{t=13}^{14} 0.05 v_{t}+\sum_{t=15}^{44} 0.08 v_{t} \\
+ & \sum_{t=45}^{46} 0.054 v_{t}+\sum_{t=47}^{48} 0.047 v_{t}
\end{aligned}
$$




\subsection{Chapter summary}

This chapter provides an optimization approach to optimal scheduling of EV charging for $\mathrm{BEV}$ users in residential areas. In particular, two categories of BEV users are considered: short-distance and medium-distance users. The main objective of the optimization models is to minimize the total cost of electricity usage while still meeting both household and BEV demands for all users. We modeled two scenarios of optimal charging: (1) night charging, (2) day-and-night charging. Two optimization models are then customized to three electricity cost structures: the liner cost function, the piecewise linear cost function and the time-of-use cost function. An analysis to compare the results of the optimization models that incorporate the different pricing structures under two charging scenarios will be provided in Chapter 5 . 


\section{CHAPTER 4: DECENTRALIZED OPTIMIZATION MODEL}

In this chapter, we discuss an alternative approach to scheduling the optimal charging profile of the BEV users in residential areas. We employ agent-based modeling techniques to develop the decentralized optimal charging scheduling model. First, we provide an overview of the decentralized approach where each BEV user actively participates to reduce their own cost of electricity usage while still meeting their daily household and BEV charging demands. Henceforth, we present the decentralized model which includes the outline of the approach, pricing incentive and the mechanisms for coordination among the BEV users.

\subsection{Overview of decentralized approach}

In Chapter 3, we have developed a centralized optimal charging schedule to coordinate the times for BEV users to charge their electric vehicles. However, in reality, the decisions from a central controller often disregard the preference of when to charge the BEV by individual users. Hence, we propose an agent-based approach which offers flexibility to users with regards to the time to charge and the duration of the charging. Under this approach, each BEV user optimizes his/her own cost of electricity usage, while being mindful about others' usage and the dynamic price of electricity at each time interval $t$. Although this approach does not necessarily yield the minimum costs for all 
households collectively, it is more likely to be accepted by public because their individual preferences are respected. Furthermore, this approach is in line with the principle of distributed computing, and can be integrated into smart software agents in smart meters (Vytelingum et al., 2010).

Here, we adopt the learning and adaptive mechanisms from a study by Vytelingum et al. (2010). Vytelingum et al. (2010) developed an adaptive storage approach via agent-based methodology. Under this strategy, each agent will change his/her storage profile daily to achieve the perceived "near-optimal" strategy. In this paper, we consider each BEV user $i$ as an agent that aims to achieve a minimum total cost of electricity daily and fulfills his/her charging demand. To accomplish this goal, each agent will have to learn and adapt his/her charging profile over time on the market. Every day, the agents adjust their charging behavior based on previous day's pricing information.

Moreover, we deem that the charging profiles of all BEV agents in the system will eventually converge to efficient charging profiles for all agents after a certain learning period. Nonetheless, several rationality assumptions have to be made. First, we presume that all agents will react rationally by adopting a charging profile that minimizes their own electricity cost. Second, we consider the charging behavior of each agent as a consequence of a price signal. 


\subsection{Decentralized optimal charging model}

We formulate a mixed integer linear programming model for the decentralized problem which considers a fixed time interval for each day composed of 48 half-hour periods. As an incentive for the agents to adjust their timing for charging, we choose to implement the linear cost function as discussed in Chapter 3. Note that this choice of linear cost function is only for illustration purpose. Thus, other cost functions can also be applied. The main objective of each agent $i$ is to minimize its total electricity costs by only charging when the price at time interval $t$ is low. Under this decentralized approach, the total load $v_{t}$ at time interval $t$ is described as the sum of household load $D_{i}^{t}$ and extra demands $x_{i}^{t}$ from each BEV agent $i$.

Furthermore, we apply the concept of "day-ahead best response" as proposed by Vytelingum et al. (2010). By doing so, we estimate the price based on yesterday's pricing information at a particular time interval $t$. Note that in this chapter, we implement the optimal day-and-night charging scenario. Hence, the previous global optimization problem which involves $n$ residential users is reduced to a local optimization problem for each user $i$. The decentralized optimal charging model is implemented and solved using GAMS/CPLEX. Below is the optimization problem that each agent $i$ solves.

$$
\operatorname{Min} \sum_{t=1}^{48} p_{t}\left(v_{t}\right) * v_{t}
$$

Subject to:

$$
v_{t}=\sum_{i=1}^{n}\left(D_{i}^{t}+x_{i}^{t}\right) \quad \forall t
$$




$$
\begin{array}{ll}
\sum_{t=1}^{48} x_{i}^{t}=d_{i} & \\
\sum_{t=17}^{36} y_{i}^{t} \geq 1 & \\
\sum_{t=17}^{36} y_{i}^{t} \leq 3 & >20 k W h \\
x_{i}^{t}=3.3 * y_{i}^{t} & \\
& \\
& \forall t \\
x_{i}^{t}=1.65 * y_{i}^{t} & \in\{17, \ldots, 36\} \\
& \forall t \\
& \in\{1, \ldots, 16\} \\
& \cup\{37, \ldots, 48\} \\
y_{i}^{t} \leq 1-y_{i}^{t+1} & \\
x_{i}^{t} \geq 0 & \forall t \\
y_{i}^{t} \in\{0,1\} & \in\{17, \ldots, 36\} \\
& \\
& \forall t
\end{array}
$$

We consider two decision variables in this optimization problem. First, we solve for $x_{i}^{t}$ which is the amount of charge for BEV agent $i$ who starts to charge for 30 minutes at time interval $t$. The second decision variable is $y_{i}^{t}$ which determines whether agent $i$ will begin his/her charging at time interval $t$ for 30 minutes.

Equation (4-1) represents the cost function that each agent $i$ is minimizing for a single day period between 12:00 am and 11:30 pm. The price $p_{t}$ is yesterday's electricity price at time interval $t$. Constraint (4-2) calculates the total electricity usage for agent $i$. Constraint (4-3) ensures that the charging requirements or demands from agent $i$ is 
fulfilled. Constraint (4-4) states that the medium-distance users are enforced to charge at least once during the day which is between $8: 00$ am to $5: 30 \mathrm{pm}$. Constraint (4-5) ensures that all users can only charge for a maximum of 3 times during the day. Constraint (4-6) represents a total charge of $3.3 \mathrm{kWh}$ assigned to the BEV user $i$ who is scheduled to charge between $8: 00$ am to $5: 30 \mathrm{pm}$. On the other hand, Constraint (4-7) states that a total of $1.65 \mathrm{kWh}$ is allocated to BEV user $i$ who is scheduled to charge from 5:30 pm to 7:30 am the following day. Lastly, Constraint (4-8) states that consecutive charging for two half-hour intervals is enforced to all users who are scheduled to charge during the day. In this work, we do not consider any storage capability from the electric vehicle. Thus, we only allow a positive charging amount which is represented by Constraint (4-9). Lastly, Constraint (4-10) specifies that $y_{i}^{t}$ is a binary decision variable.

\subsection{Coordination mechanisms among agents}

The fundamental idea behind the coordination of the BEV agents in the system is through the agents' learning process. In particular, every day, agent only minimizes his/her own electricity cost in scheduling his/her electric vehicle charging based on yesterday's electricity price at each time interval $t$. Note that yesterday's electricity price is evaluated based on the total load of all users. This way, implicitly each agent makes charging decision based on others' decision from the previous day. This can be viewed as a form of game that all users play against each other to ensure the lowest utility (electricity) cost. Since this is a rather distributed process, it may take numerous iterations to converge. Such an iterative process can be interpreted as a "learning" process on the market for all agents. Particularly, at each iteration or on each "learning" day, 
agent $i$ decides his/her charging profile $x_{i}^{t}$ to achieve the lowest electricity cost possible by solving problem (4-1) to (4-10). Again, implicitly, this locally optimal charging profile $x_{i}^{t}$ depends on the charging decisions from other agents, at least from yesterday. All agents solve these problems spontaneously. The optimal charging profiles $x_{i}^{t}$ from all agents for today are used to evaluate today's total load $v_{t}$ and predict tomorrow's price at any time interval $t$. Then, the next iteration carries on with this price as $p_{t}$ in the objective function (4-1). This iterative process terminates when the charging profiles for all users from day to day do not change, or in other words, when they converge. A flow chart of this process is shown in Figure 3.

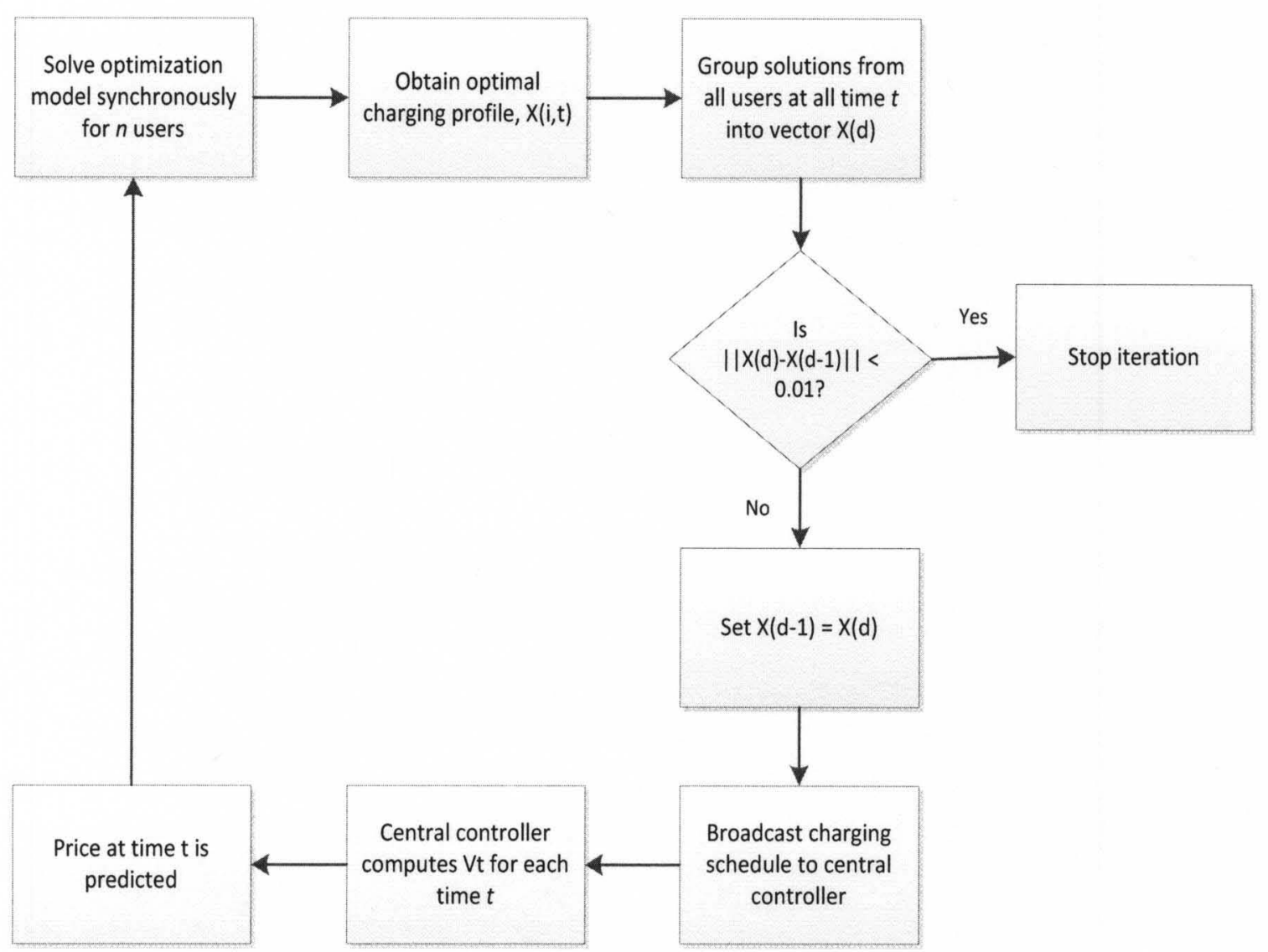

Figure 3: Algorithm of the decentralized model 
The outline of the decentralized model is described below.

1. Step 0: Initialization. Day, $d=0 ; p_{t}=\sum_{i=1}^{n} D_{i}^{t}$; initial charging profile $X(0)=$ 0 for all users and all time interval $t$.

2. Step 1: Each agent solves synchronously their own scheduling problem (4-1) to (4-10) by fixing the decision from other agents and using previous day's price $p_{t}$ at time interval $t$.

3. Step 2: If $d \geq 2$, then $X(d-1)=X(d)$ (keep a record of yesterday's charging profile). All solutions $x_{i}^{t}$ from Step 1 are grouped into today's charging profile vector $X(d)=x_{i}^{t}$ where $1 \leq t \leq 48,1 \leq i \leq n$.

4. Step 3: All agents will broadcast their usage to the central controller. Calculate $v_{t}=\sum_{i=1}^{n}\left(D_{i}^{t}+x(d)_{i}^{t}\right.$ and $p_{t}=p\left(v_{t}\right)$

5. Step 4: If $\|X(d)-X(d-1)\|<\alpha$, then stop; otherwise, $d=d+1$, go to step 1 (when no further improvements are made, each agent settles with the perceived "near-optimal" strategy).

In Step 0 , we initialize the total load $v_{t}$ by only considering the agent's household demand $D_{i}^{t}$. Therefore, the initial price for time interval $t$ is only due to agent's household demands. In Step 1, we solve the scheduling problem synchronously for $n$ BEV agents. The solutions for all $n \mathrm{BEV}$ agents are kept as today's charging profiles, and later they are announced to the central controller as listed in Step 3. In Step 3, the algorithm in the central controller calculates today's total load $v_{t}$ for each time interval, and then predicts tomorrow's electricity price. Then, we move onto the next day living through the same process by returning to Step 1. During this process, we check the charging profiles $x_{i}^{t}$ for 
all users between two consecutive days. If there is negligible difference between the two, we consider no further learning is needed, i.e., the agents have achieved their "nearoptimal" charging strategies. Therefore, we terminate the process. Note that in Step 2, for technical convenience, we group all individual's charging profiles $x_{i}^{t}(1 \leq t \leq 48,1 \leq$ $i \leq n)$ to a vector $X(\mathrm{~d})$. Then, in Step 4, we calculate the norm of the vector $X(d)-$ $X(d-1)$, which represents the difference between charging profiles on two consecutive days. Here, we use the $L_{2}$ norm defined below in Equation (4-11).

$$
\frac{\|X(d)-X(d-1)\|:=}{\sqrt{\sum_{i}\left(x_{i, d}^{1}-x_{i, d-1}^{1}\right)^{2}+. .+\left(x_{i, d}^{48}-x_{i, d-1}^{48}\right)^{2}}}
$$

Additionally, in Step 4, $\alpha$ is the threshold value for determining if charging profiles $x_{i}^{t}$ on two consecutive days are close enough. In our numerical implementations, we use $\alpha=0.01$.

\subsection{Chapter summary}

A decentralized optimal charging model specifically for night scenario is presented in this chapter. The system was modeled using a mixed integer linear program. In a decentralized model, each BEV agent $i$ solves his/her own local scheduling problem by learning and adapting his/her charging profile based on other agents' charging decisions over a learning period. Pricing information is used as an incentive to motivate the agents to adjust their timing to charge. Consequently, each agent will alter the charging period based on previous day's price at the particular time. We conclude that the 
agents have obtained a "nearly-optimal" strategy when there are no further improvements seen in the solution. 


\section{CHAPTER 5: NUMERICAL RESULTS}

In this chapter, we report numerical results for the optimization models described in Chapters 3 and 4. All optimization models are implemented using GAMS, and we used CPLEX 10.2 to solve them. We begin with the uncontrolled charging scenario which is taken as a reference case. Next, we present the results of centralized optimization models that explore the effect of implementing different cost functions on the charging schedule of BEV users under two scenarios, i.e., night-only and day-and-night charging scenarios. The two cost functions that we implement in our work are the linear cost and time-of-use pricing structure. Subsequently, we provide the results for the decentralized optimization model which aims to minimize the cost for each individual user.

For all analysis, we vary the total number of users $n$ for the following values: 10 , 50,100 and 200. For each number of $n$ users, we tested for several penetration rates of medium-distance users using values of $20 \%, 35 \%$ and $50 \%$. We run a total of 15 instances for each value $n$ with 5 instances for different penetration rates and calculated the total electricity cost and the peak-to-average ratio (PAR). Particularly, PAR is calculated according to the formula shown in Equation 5-1.

$$
P A R=\frac{\text { Maximum load }}{\text { Average load }}
$$


Table 3 summarizes the problem sizes for the centralized models that we study under a linear cost function. The results in Table 3 show the average number of continuous variables, average number of binary variables and total number of constraints for different penetration rates of medium-distance users, i.e., $20 \%, 35 \%$ and $50 \%$. For instance, the centralized model under a night-only charging scenario for 10 users at $20 \%$ penetration rate contains 132 continuous variables and 132 binary variables. A total of 1508 constraints are included to solve the night-only charging scenario for 10 users at $20 \%$ penetration rate.

Correspondingly, Table 4 displays the problem sizes for the centralized models under time-of-use pricing structure. On the other hand, Table 5 displays the problem sizes for the decentralized model under a linear cost function. Overall, we observe that the problem size increases as we increase the number of users $n$ from 10 to 200. Similarly, the problem size also increases with an increasing penetration rate of medium-distance users.

Table 3: Problem sizes for centralized models (linear cost)

\begin{tabular}{|c|c|c|c|c|c|}
\hline $\begin{array}{l}\text { Charging } \\
\text { Scenario }\end{array}$ & $\begin{array}{c}\text { \# of } \\
\text { users, n }\end{array}$ & $\begin{array}{l}\text { Penetration } \\
\text { rate of med- } \\
\text { distance } \\
\text { users }\end{array}$ & $\begin{array}{c}\text { Ave. Total \# } \\
\text { of } \\
\text { continuous } \\
\text { variables }\end{array}$ & $\begin{array}{l}\text { Ave. Total \# } \\
\text { of binary } \\
\text { variables }\end{array}$ & $\begin{array}{c}\text { Total \# of } \\
\text { constraints }\end{array}$ \\
\hline \multirow{9}{*}{ Night-only } & \multirow{3}{*}{10} & $20 \%$ & 132 & 132 & \multirow{3}{*}{1508} \\
\hline & & $35 \%$ & 138 & 138 & \\
\hline & & $50 \%$ & 150 & 150 & \\
\hline & \multirow{3}{*}{50} & $20 \%$ & 660 & 660 & \multirow{3}{*}{7348} \\
\hline & & $35 \%$ & 702 & 702 & \\
\hline & & $50 \%$ & 750 & 750 & \\
\hline & \multirow{3}{*}{100} & $20 \%$ & 1320 & 1320 & \multirow{3}{*}{14648} \\
\hline & & $35 \%$ & 1410 & 1410 & \\
\hline & & $50 \%$ & 1500 & 1500 & \\
\hline
\end{tabular}




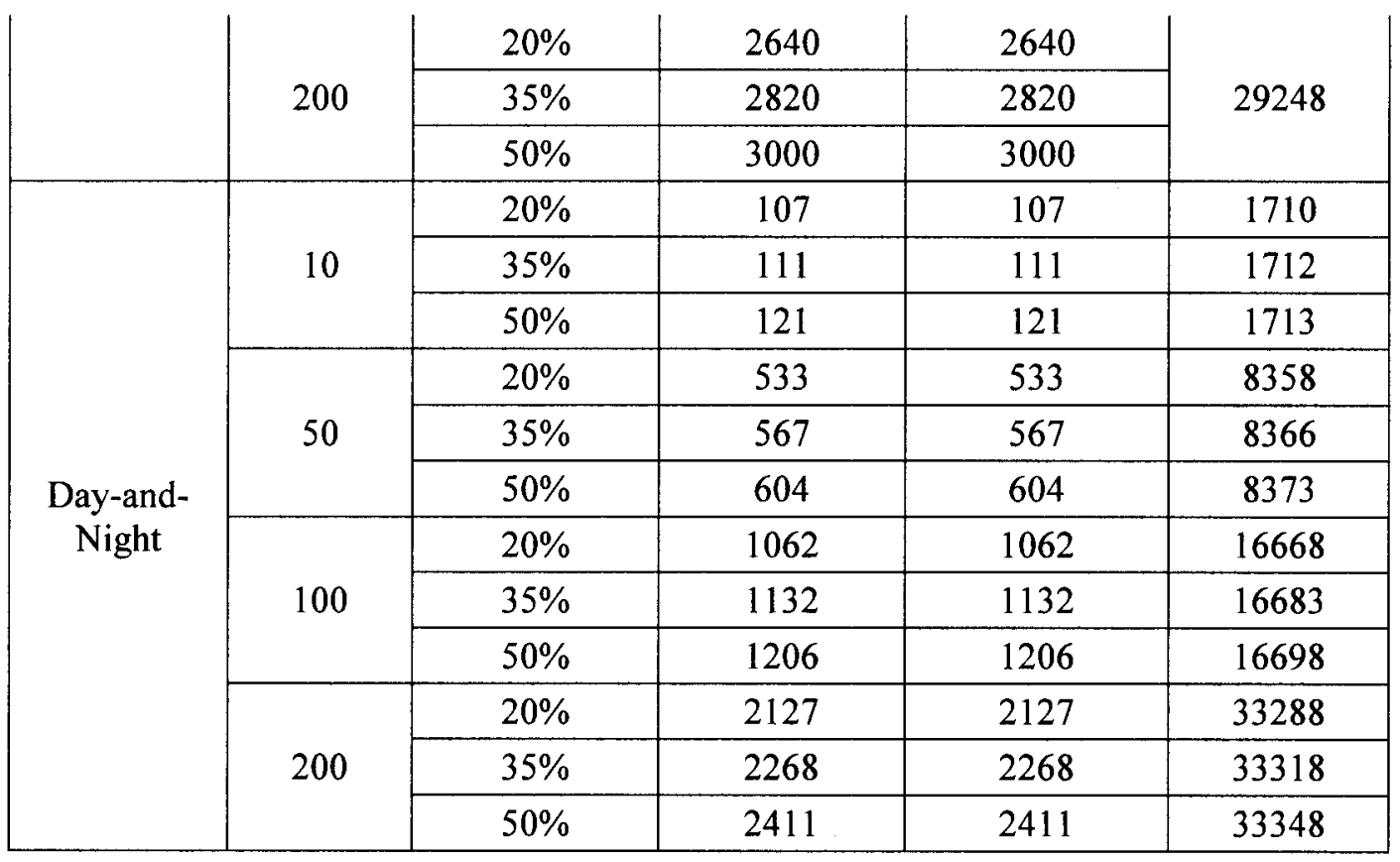

Table 4: Problem sizes for centralized models (TOU)

\begin{tabular}{|c|c|c|c|c|c|}
\hline $\begin{array}{l}\text { Charging } \\
\text { Scenario }\end{array}$ & $\begin{array}{c}\text { \# of users, } \\
\text { n }\end{array}$ & $\begin{array}{l}\text { Penetration } \\
\text { rate of med- } \\
\text { distance } \\
\text { users }\end{array}$ & $\begin{array}{c}\text { Ave. Total \# } \\
\text { of } \\
\text { continuous } \\
\text { variables }\end{array}$ & $\begin{array}{c}\text { Ave. Total \# } \\
\text { of binary } \\
\text { variables }\end{array}$ & $\begin{array}{l}\text { Total \# of } \\
\text { constraints }\end{array}$ \\
\hline \multirow{12}{*}{ Night-only } & \multirow{3}{*}{10} & $20 \%$ & 132 & 132 & \multirow{3}{*}{1508} \\
\hline & & $35 \%$ & 138 & 138 & \\
\hline & & $50 \%$ & 150 & 150 & \\
\hline & \multirow{3}{*}{50} & $20 \%$ & 660 & 660 & \multirow{3}{*}{7348} \\
\hline & & $35 \%$ & 702 & 702 & \\
\hline & & $50 \%$ & 750 & 750 & \\
\hline & \multirow{3}{*}{100} & $20 \%$ & 1320 & 1320 & \multirow{3}{*}{14648} \\
\hline & & $35 \%$ & 1410 & 1410 & \\
\hline & & $50 \%$ & 1500 & 1500 & \\
\hline & \multirow{3}{*}{200} & $20 \%$ & 2640 & 2640 & \multirow{3}{*}{29248} \\
\hline & & $35 \%$ & 2820 & 2820 & \\
\hline & & $50 \%$ & 3000 & 3000 & \\
\hline \multirow{5}{*}{$\begin{array}{c}\text { Day-and- } \\
\text { Night }\end{array}$} & \multirow{3}{*}{10} & $20 \%$ & 130 & 130 & 1710 \\
\hline & & $35 \%$ & 135 & 135 & 1712 \\
\hline & & $50 \%$ & 145 & 145 & 1713 \\
\hline & \multirow{2}{*}{50} & $20 \%$ & 650 & 650 & 8358 \\
\hline & & $35 \%$ & 685 & 685 & 8366 \\
\hline
\end{tabular}




\begin{tabular}{|c|c|c|c|c|c|} 
& $50 \%$ & 725 & 725 & 8373 \\
\hline \multirow{3}{*}{100} & $20 \%$ & 1300 & 1300 & 16668 \\
\cline { 2 - 5 } & $35 \%$ & 1375 & 1375 & 16683 \\
\cline { 2 - 5 } & $50 \%$ & 1450 & 1450 & 16698 \\
\hline \multirow{3}{*}{200} & $20 \%$ & 2600 & 2600 & 33288 \\
\cline { 2 - 5 } & $35 \%$ & 2750 & 2750 & 33318 \\
\cline { 2 - 5 } & & $50 \%$ & 2900 & 2900 & 33348 \\
\hline
\end{tabular}

Table 5: Problem sizes for decentralized model

\begin{tabular}{|c|c|c|c|c|c|}
\hline $\begin{array}{l}\text { Charging } \\
\text { Scenario }\end{array}$ & $\begin{array}{c}\text { \# of users, } \\
\text { n }\end{array}$ & $\begin{array}{l}\text { Penetration } \\
\text { rate of med- } \\
\text { distance } \\
\text { users }\end{array}$ & $\begin{array}{c}\text { Total \# of } \\
\text { continuous } \\
\text { variables }\end{array}$ & $\begin{array}{c}\text { Total \# of } \\
\text { binary } \\
\text { variables }\end{array}$ & $\begin{array}{l}\text { Total \# of } \\
\text { constraints }\end{array}$ \\
\hline \multirow{12}{*}{$\begin{array}{c}\text { Day-and- } \\
\text { Night }\end{array}$} & \multirow{3}{*}{10} & $20 \%$ & 126 & 126 & 2142 \\
\hline & & $35 \%$ & 129 & 129 & 2144 \\
\hline & & $50 \%$ & 145 & 145 & 2145 \\
\hline & \multirow{3}{*}{50} & $20 \%$ & 630 & 630 & 10710 \\
\hline & & $35 \%$ & 668 & 668 & 10718 \\
\hline & & $50 \%$ & 725 & 725 & 10725 \\
\hline & \multirow{3}{*}{100} & $20 \%$ & 1260 & 1260 & 21420 \\
\hline & & $35 \%$ & 1375 & 1375 & 21435 \\
\hline & & $50 \%$ & 1450 & 1450 & 21450 \\
\hline & \multirow{3}{*}{200} & $20 \%$ & 2520 & 2520 & 42840 \\
\hline & & $35 \%$ & 2750 & 2750 & 42870 \\
\hline & & $50 \%$ & 2900 & 2900 & 42900 \\
\hline
\end{tabular}

\subsection{Results for uncontrolled charging scenario}

\subsubsection{Results for uncontrolled charging under linear cost function}

This section presents the results of uncontrolled charging scenario for BEV users in residential areas under the linear cost function introduced in Section 3.4. Table 6 displays the performance measures for uncontrolled charging for 10 users. From Table 6, 
we observe that the average cost is $\$ 241.17$ at $20 \%$ penetration rate of medium-distance users. The average PAR, on the other hand, is calculated to be 2.32. As we increase the medium-distance penetration rate to $35 \%$, the average cost increases slightly to $\$ 245.19$ while the average PAR decreases to 2.27 . Finally, at $50 \%$ penetration rate, the average total cost is $\$ 256.65$ for 10 users with a PAR of 2.22. From the results in Table 6, we notice that as the penetration rate increases, the total electricity cost increases. However, the PAR decreases as we increase the penetration rate of medium-distance users.

Table 6: Results for uncontrolled charging under linear cost (10 users)

\begin{tabular}{|c|c|c|c|c|c|c|}
\hline \multirow[b]{3}{*}{ Instance } & \multicolumn{6}{|c|}{ Penetration rate of medium-distance users } \\
\hline & \multicolumn{2}{|c|}{$20 \%$} & \multicolumn{2}{|c|}{$35 \%$} & \multicolumn{2}{|c|}{$50 \%$} \\
\hline & $\begin{array}{c}\text { Total } \\
\text { Electricity } \\
\text { Cost }\end{array}$ & PAR & $\begin{array}{c}\text { Total } \\
\text { Electricity } \\
\text { Cost }\end{array}$ & PAR & $\begin{array}{c}\text { Total } \\
\text { Electricity } \\
\text { Cost }\end{array}$ & PAR \\
\hline 1 & $\$ 237.28$ & 2.31 & $\$ 248.61$ & 2.26 & $\$ 256.21$ & 2.23 \\
\hline 2 & $\$ 240.63$ & 2.32 & $\$ 244.20$ & 2.29 & $\$ 257.17$ & 2.21 \\
\hline 3 & $\$ 240.59$ & 2.34 & $\$ 246.15$ & 2.28 & $\$ 254.72$ & 2.25 \\
\hline 4 & $\$ 243.65$ & 2.32 & $\$ 243.75$ & 2.26 & $\$ 256.11$ & 2.20 \\
\hline 5 & $\$ 243.69$ & 2.33 & $\$ 243.26$ & 2.28 & $\$ 259.07$ & 2.19 \\
\hline$\overline{\text { AVERAGE }}$ & $\$ 241.17$ & 2.32 & $\$ 245.19$ & 2.27 & $\$ 256.65$ & 2.22 \\
\hline
\end{tabular}

We find similar observations from the numerical results of 50,100 and 200 users, as shown in Tables 7,8 and 9 , respectively. In particular, as the penetration rate of medium distance users increases, the overall electricity cost increases and the PAR values decreases. Additionally, we note that the average PAR for the penetration rates of $20 \%$, $35 \%$ and $50 \%$ from Tables 7,8 and 9 are almost the same for 10, 50, 100 and 200 residential users considered in the model. 
Table 7: Results for uncontrolled charging under linear cost (50 users)

\begin{tabular}{|c|c|c|c|c|c|c|}
\hline \multirow{3}{*}{ Instance } & \multicolumn{3}{|c|}{ Penetration rate of medium-distance users } \\
\cline { 2 - 7 } & \multicolumn{2}{|c|}{$\mathbf{2 0 \%}$} & \multicolumn{2}{c|}{$\mathbf{3 5 \%}$} & \multicolumn{2}{c|}{$\mathbf{5 0 \%}$} \\
\cline { 2 - 7 } & $\begin{array}{c}\text { Total } \\
\text { Electricity } \\
\text { Cost }\end{array}$ & PAR & $\begin{array}{c}\text { Total } \\
\text { Electricity } \\
\text { Cost }\end{array}$ & PAR & $\begin{array}{c}\text { Total } \\
\text { Electricity } \\
\text { Cost }\end{array}$ & PAR \\
\hline 1 & $\$ 5,038.20$ & 2.32 & $\$ 5,304.81$ & 2.26 & $\$ 5,474.25$ & 2.23 \\
\hline 2 & $\$ 5,140.60$ & 2.32 & $\$ 5,259.75$ & 2.27 & $\$ 5,464.34$ & 2.21 \\
\hline 3 & $\$ 5,157.49$ & 2.33 & $\$ 5,308.26$ & 2.27 & $\$ 5,451.59$ & 2.25 \\
\hline 4 & $\$ 5,149.95$ & 2.32 & $\$ 5,280.16$ & 2.27 & $\$ 5,417.81$ & 2.20 \\
\hline 5 & $\$ 5,101.72$ & 2.31 & $\$ 5,211.09$ & 2.26 & $\$ 5,476.72$ & 2.19 \\
\hline AVERAGE & $\$ 5,117.59$ & 2.32 & $\$ 5,272.81$ & 2.27 & $\$ 5,456.94$ & 2.22 \\
\hline
\end{tabular}

Table 8: Results for uncontrolled charging under linear cost (100 users)

\begin{tabular}{|c|c|c|c|c|c|c|}
\hline \multirow{3}{*}{ Instance } & \multicolumn{6}{|c|}{ Penetration rate of medium-distance users } \\
\cline { 2 - 7 } & \multicolumn{2}{|c|}{$\mathbf{2 0 \%}$} & \multicolumn{2}{c|}{$\mathbf{3 5 \%}$} & \multicolumn{2}{|c|}{$\mathbf{5 0 \%}$} \\
\cline { 2 - 7 } & $\begin{array}{c}\text { Total } \\
\text { Electricity } \\
\text { Cost }\end{array}$ & PAR & $\begin{array}{c}\text { Total } \\
\text { Electricity } \\
\text { Cost }\end{array}$ & PAR & $\begin{array}{c}\text { Total } \\
\text { Electricity } \\
\text { Cost }\end{array}$ & PAR \\
\hline 1 & $\$ 19,993.63$ & 2.31 & $\$ 20,456.85$ & 2.26 & $\$ 21,374.48$ & 2.21 \\
\hline 2 & $\$ 20,182.42$ & 2.32 & $\$ 20,598.38$ & 2.27 & $\$ 21,365.14$ & 2.21 \\
\hline 3 & $\$ 19,939.27$ & 2.32 & $\$ 20,731.07$ & 2.26 & $\$ 21,303.35$ & 2.21 \\
\hline 4 & $\$ 20,007.30$ & 2.31 & $\$ 20,749.53$ & 2.26 & $\$ 21,230.80$ & 2.20 \\
\hline 5 & $\$ 19,991.26$ & 2.32 & $\$ 20,663.18$ & 2.26 & $\$ 21,433.99$ & 2.21 \\
\hline AVERAGE & $\$ 20,022.78$ & 2.31 & $\$ 20,639.80$ & 2.26 & $\$ 21,341.55$ & 2.21 \\
\hline
\end{tabular}

Table 9: Results for uncontrolled charging under linear cost (200 users)

\begin{tabular}{|c|c|c|c|c|c|c|}
\hline \multirow{3}{*}{ Instance } & \multicolumn{5}{|c|}{ Penetration rate of medium-distance users } \\
\cline { 2 - 7 } & \multicolumn{2}{|c|}{$\mathbf{2 0 \%}$} & \multicolumn{2}{c|}{$\mathbf{3 5 \%}$} & \multicolumn{2}{|c|}{$\mathbf{5 0 \%}$} \\
\cline { 2 - 7 } & $\begin{array}{c}\text { Total } \\
\text { Electricity } \\
\text { Cost }\end{array}$ & PAR & $\begin{array}{c}\text { Total } \\
\text { Electricity } \\
\text { Cost }\end{array}$ & PAR & $\begin{array}{c}\text { Total } \\
\text { Electricity } \\
\text { Cost }\end{array}$ & PAR \\
\hline 1 & $\$ 79,034.86$ & 2.32 & $\$ 81,502.89$ & 2.26 & $\$ 84,520.76$ & 2.21 \\
\hline 2 & $\$ 79,487.18$ & 2.33 & $\$ 81,421.52$ & 2.26 & $\$ 84,386.76$ & 2.21 \\
\hline 3 & $\$ 79,255.07$ & 2.32 & $\$ 81,907.31$ & 2.26 & $\$ 84,799.95$ & 2.21 \\
\hline 4 & $\$ 79,044.15$ & 2.31 & $\$ 81,548.00$ & 2.25 & $\$ 84,984.76$ & 2.21 \\
\hline 5 & $\$ 79,344.08$ & 2.32 & $\$ 81,967.88$ & 2.26 & $\$ 84,696.58$ & 2.21 \\
\hline AVERAGE & $\$ 79,233.07$ & 2.32 & $\$ 81,669.52$ & 2.26 & $\$ 84,677.76$ & 2.21 \\
\hline
\end{tabular}


In addition, Figure 4 depicts the residential total load profile (including both the regular household usage as well as EV charging) for 10 users with uncontrolled EV charging (from 6:00 pm to 7:00 am). The load profiles for user sizes of 50, 100 and 200 are similar. From Figure 4, we observe that there is a significant increase in energy demand from $6: 00 \mathrm{pm}(t=37)$ to $11: 30 \mathrm{pm}(t=48)$ compared to other time intervals. The significant increase is due to simultaneous starting time for EV charging from all users.

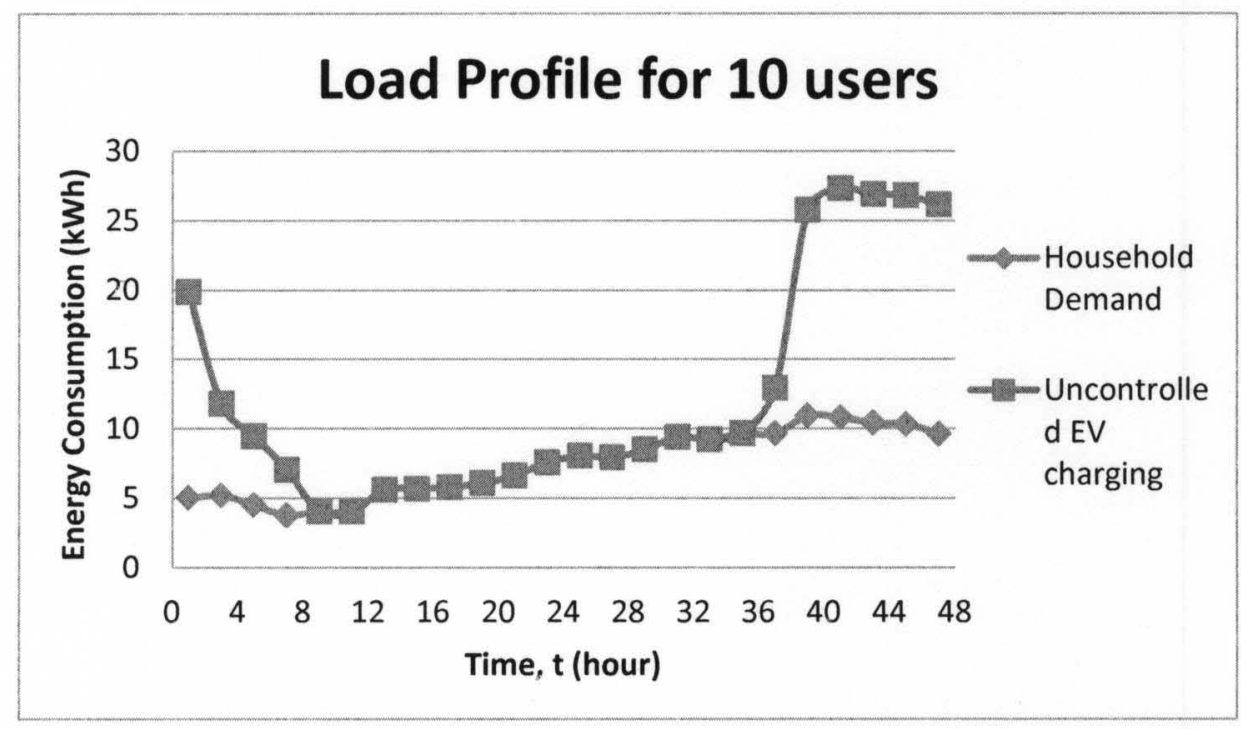

Figure 4: Load profile with uncontrolled EV charging under linear cost

\subsubsection{Results for uncontrolled charging under time-of-use (TOU) cost}

Table 10 displays the results for uncontrolled EV charging under the time-of-use pricing structure, which we introduced in Section 3.5, for 10 users. The average total electricity costs are $\$ 38.99, \$ 39.42$ and $\$ 40.33$ for the penetrate rate of medium-distance users being $20 \%, 35 \%$ and $50 \%$, respectively. Collectively, Table 10 suggests that as the penetration rate of medium distance users increases, the total cost increases while the 
average PAR decreases. Similar observations can be made for 50,100 and 200 users. These observations are consistent with those obtained under the linear cost function.

Table 10: Results for uncontrolled charging under TOU (10 users)

\begin{tabular}{|c|c|c|c|c|c|c|}
\hline \multirow[b]{3}{*}{ Instance } & \multicolumn{6}{|c|}{ Penetration rate of medium-distance users } \\
\hline & \multicolumn{2}{|c|}{$20 \%$} & \multicolumn{2}{|c|}{$35 \%$} & \multicolumn{2}{|c|}{$50 \%$} \\
\hline & $\begin{array}{c}\text { Total } \\
\text { Electricity } \\
\text { Cost }\end{array}$ & PAR & $\begin{array}{c}\text { Total } \\
\text { Electricity } \\
\text { Cost }\end{array}$ & PAR & \begin{tabular}{|c|} 
Total \\
Electricity \\
Cost
\end{tabular} & PAR \\
\hline 1 & $\$ 38.69$ & 2.31 & $\$ 39.84$ & 2.26 & $\$ 40.34$ & 2.23 \\
\hline 2 & $\$ 38.72$ & 2.32 & $\$ 39.22$ & 2.29 & $\$ 40.30$ & 2.21 \\
\hline 3 & $\$ 39.00$ & 2.34 & $\$ 39.76$ & 2.28 & $\$ 40.41$ & 2.25 \\
\hline 4 & $\$ 39.30$ & 2.32 & $\$ 39.25$ & 2.26 & $\$ 40.18$ & 2.20 \\
\hline 5 & $\$ 39.26$ & 2.33 & $\$ 39.04$ & 2.28 & $\$ 40.41$ & 2.19 \\
\hline AVERAGE & $\$ 38.99$ & 2.32 & $\$ 39.42$ & 2.27 & $\$ 40.33$ & 2.22 \\
\hline
\end{tabular}

Table 11: Results for uncontrolled charging under TOU (50 users)

\begin{tabular}{|c|c|c|c|c|c|c|}
\hline \multirow{3}{*}{ Instance } & \multicolumn{5}{|c|}{ Penetration rate of medium-distance users } \\
\cline { 2 - 7 } & \multicolumn{2}{|c|}{$\mathbf{2 0 \%}$} & \multicolumn{2}{c|}{$\mathbf{3 5 \%}$} & \multicolumn{2}{c|}{$\mathbf{5 0 \%}$} \\
\cline { 2 - 7 } & $\begin{array}{c}\text { Total } \\
\text { Electricity } \\
\text { Cost }\end{array}$ & PAR & $\begin{array}{c}\text { Total } \\
\text { Electricity } \\
\text { Cost }\end{array}$ & PAR & $\begin{array}{c}\text { Total } \\
\text { Electricity } \\
\text { Cost }\end{array}$ & PAR \\
\hline 1 & $\$ 191.65$ & 2.32 & $\$ 197.53$ & 2.26 & $\$ 200.42$ & 2.20 \\
\hline 2 & $\$ 194.36$ & 2.32 & $\$ 196.84$ & 2.27 & $\$ 200.25$ & 2.21 \\
\hline 3 & $\$ 194.72$ & 2.33 & $\$ 197.83$ & 2.27 & $\$ 200.04$ & 2.20 \\
\hline 4 & $\$ 194.04$ & 2.32 & $\$ 196.22$ & 2.27 & $\$ 199.67$ & 2.20 \\
\hline 5 & $\$ 193.70$ & 2.31 & $\$ 195.70$ & 2.26 & $\$ 200.32$ & 2.22 \\
\hline AVERAGE & $\$ 193.70$ & 2.32 & $\$ 196.82$ & 2.27 & $\$ 200.14$ & 2.21 \\
\hline
\end{tabular}


Table 12: Results for uncontrolled charging under TOU (100 users)

\begin{tabular}{|c|c|c|c|c|c|c|}
\hline \multirow{2}{*}{ Instance } & \multicolumn{5}{|c|}{ Penetration rate of medium-distance users } \\
\cline { 2 - 7 } & \multicolumn{2}{|c|}{$\mathbf{2 0 \%}$} & \multicolumn{2}{c|}{$\mathbf{3 5 \%}$} & \multicolumn{2}{c|}{$\mathbf{5 0 \%}$} \\
\cline { 2 - 7 } & $\begin{array}{c}\text { Total } \\
\text { Electricity } \\
\text { Cost }\end{array}$ & PAR & $\begin{array}{c}\text { Total } \\
\text { Electricity } \\
\text { Cost }\end{array}$ & PAR & $\begin{array}{c}\text { Total } \\
\text { Electricity } \\
\text { Cost }\end{array}$ & PAR \\
\hline 1 & $\$ 387.59$ & 2.31 & $\$ 392.06$ & 2.26 & $\$ 400.10$ & 2.21 \\
\hline 2 & $\$ 388.20$ & 2.32 & $\$ 393.69$ & 2.27 & $\$ 400.33$ & 2.21 \\
\hline 3 & $\$ 385.97$ & 2.32 & $\$ 393.53$ & 2.26 & $\$ 400.29$ & 2.21 \\
\hline 4 & $\$ 387.30$ & 2.31 & $\$ 395.00$ & 2.26 & $\$ 399.45$ & 2.20 \\
\hline 5 & $\$ 386.45$ & 2.32 & $\$ 243.26$ & 2.26 & $\$ 400.73$ & 2.21 \\
\hline AVERAGE & $\$ 387.10$ & 2.31 & $\$ 363.51$ & 2.26 & $\$ 400.18$ & 2.21 \\
\hline
\end{tabular}

Table 13: Results for uncontrolled charging under TOU (200 users)

\begin{tabular}{|c|c|c|c|c|c|c|}
\hline \multirow[b]{3}{*}{ Instance } & \multicolumn{6}{|c|}{ Penetration rate of medium-distance users } \\
\hline & \multicolumn{2}{|c|}{$20 \%$} & \multicolumn{2}{|c|}{$35 \%$} & \multicolumn{2}{|c|}{$\mathbf{5 0 \%}$} \\
\hline & $\begin{array}{c}\text { Total } \\
\text { Electricity } \\
\text { Cost }\end{array}$ & PAR & $\begin{array}{c}\text { Total } \\
\text { Electricity } \\
\text { Cost }\end{array}$ & PAR & $\begin{array}{c}\text { Total } \\
\text { Electricity } \\
\text { Cost }\end{array}$ & PAR \\
\hline 1 & $\$ 773.24$ & 2.32 & $\$ 786.35$ & 2.26 & $\$ 800.28$ & 2.21 \\
\hline 2 & $\$ 775.82$ & 2.33 & $\$ 786.12$ & 2.26 & $\$ 800.54$ & 2.21 \\
\hline 3 & $\$ 774.21$ & 2.32 & $\$ 788.21$ & 2.26 & $\$ 800.91$ & 2.21 \\
\hline 4 & $\$ 772.47$ & 2.31 & $\$ 787.76$ & 2.25 & $\$ 803.27$ & 2.21 \\
\hline 5 & $\$ 774.51$ & 2.32 & $\$ 243.26$ & 2.26 & $\$ 800.61$ & 2.21 \\
\hline AVERAGE & $\$ 774.05$ & 2.32 & $\$ 678.34$ & 2.26 & $\$ 801.12$ & 2.21 \\
\hline
\end{tabular}

\subsection{Results for centralized optimal charging models}

In this section, we present the numerical results of the centralized optimal charging models under two cost functions: linear cost function and time-of-use pricing structure. 


\subsubsection{Results under linear cost function}

This section focuses on the numerical results for centralized optimization models under two scenarios. First, we study the scenario when only night charging (between 6:00 pm to 7:00 am the following day) is allowed. Second, we provide the results for day-andnight charging scenario.

\subsubsection{Results for optimal charging with night charging only}

Table 14: Results for night charging model under linear cost (10 users)

\begin{tabular}{|c|c|c|c|c|c|c|}
\hline \multirow[b]{3}{*}{ Instance } & \multicolumn{6}{|c|}{ Penetration rate of medium-distance users } \\
\hline & \multicolumn{2}{|c|}{$20 \%$} & \multicolumn{2}{|c|}{$35 \%$} & \multicolumn{2}{|c|}{$50 \%$} \\
\hline & $\begin{array}{c}\text { Total } \\
\text { Electricity } \\
\text { Cost }\end{array}$ & PAR & $\begin{array}{c}\text { Total } \\
\text { Electricity } \\
\text { Cost }\end{array}$ & PAR & $\begin{array}{c}\text { Total } \\
\text { Electricity } \\
\text { Cost }\end{array}$ & PAR \\
\hline 1 & $\$ 194.25$ & 1.34 & $\$ 201.45$ & 1.49 & $\$ 215.68$ & 1.39 \\
\hline 2 & $\$ 195.05$ & 1.36 & $\$ 201.51$ & 1.35 & $\$ 214.90$ & 1.37 \\
\hline 3 & $\$ 194.62$ & 1.40 & $\$ 202.39$ & 1.36 & $\$ 215.90$ & 1.44 \\
\hline 4 & $\$ 195.17$ & 1.37 & $\$ 201.99$ & 1.49 & $\$ 215.61$ & 1.36 \\
\hline 5 & $\$ 195.68$ & 1.34 & $\$ 201.04$ & 1.36 & $\$ 215.59$ & 1.37 \\
\hline AVERAGE & $\$ 194.96$ & 1.36 & $\$ 201.67$ & 1.41 & $\$ 215.53$ & 1.39 \\
\hline
\end{tabular}

Table 14 shows the results for the optimal night charging scenario under linear cost function for 10 users. From the numerical results shown in Table 14, the average total cost at $20 \%$ penetration rate is $\$ 194.96$ with an average PAR of 1.36 . At $35 \%$ penetration rate, the average total electricity cost increases to $\$ 201.67$ with a calculated PAR value of 1.41. As we further increase the penetration rate to $50 \%$, the average total cost for 10 users is $\$ 215.53$ with an average PAR of 1.39 . Although the PAR increases slightly when the penetration rate increases to $35 \%$ for 10 users, overall results from 
variation of 50, 100 and 200 users show that the PAR decreases as we increases the penetration rate. These results indicate that the penetration rate has some positive impact on both the total cost and PAR. We notice similar observations for performance measures for 50, 100 and 200 users shown in Tables 15,16 and 17, respectively.

Table 15: Results for night charging model under linear cost (50 users)

\begin{tabular}{|c|c|c|c|c|c|c|}
\hline \multirow{3}{*}{ Instance } & \multicolumn{4}{|c|}{ Penetration rate of medium-distance users } \\
\cline { 2 - 7 } & \multicolumn{2}{|c|}{$\mathbf{2 0 \%}$} & \multicolumn{2}{c|}{$\mathbf{3 5 \%}$} & \multicolumn{2}{c|}{$\mathbf{5 0 \%}$} \\
\cline { 2 - 7 } & $\begin{array}{c}\text { Total } \\
\text { Electricity } \\
\text { Cost }\end{array}$ & PAR & $\begin{array}{c}\text { Total } \\
\text { Electricity } \\
\text { Cost }\end{array}$ & PAR & $\begin{array}{c}\text { Total } \\
\text { Electricity } \\
\text { Cost }\end{array}$ & PAR \\
\hline 1 & $\$ 3,994.42$ & 1.31 & $\$ 4,232.32$ & 1.30 & $\$ 4,510.11$ & 1.32 \\
\hline 2 & $\$ 4,013.46$ & 1.31 & $\$ 4,223.27$ & 1.33 & $\$ 4,482.31$ & 1.35 \\
\hline 3 & $\$ 4,002.14$ & 1.31 & $\$ 4,231.74$ & 1.30 & $\$ 4,497.39$ & 1.32 \\
\hline 4 & $\$ 4,009.71$ & 1.31 & $\$ 4,233.11$ & 1.30 & $\$ 4,491.37$ & 1.35 \\
\hline 5 & $\$ 4,014.71$ & 1.34 & $\$ 4,216.71$ & 1.30 & $\$ 4,498.90$ & 1.32 \\
\hline AVERAGE & $\$ 4,006.89$ & 1.31 & $\$ 4,227.43$ & 1.31 & $\$ 4,496.02$ & 1.33 \\
\hline
\end{tabular}

Table 16: Results for night charging model under linear cost (100 users)

\begin{tabular}{|c|c|c|c|c|c|c|}
\hline \multirow{3}{*}{ Instance } & \multicolumn{4}{|c|}{ Penetration rate of medium-distance users } \\
\cline { 2 - 7 } & \multicolumn{2}{|c|}{$\mathbf{2 0 \%}$} & \multicolumn{2}{c|}{$\mathbf{3 5 \%}$} & \multicolumn{2}{c|}{$\mathbf{5 0 \%}$} \\
\cline { 2 - 8 } & $\begin{array}{c}\text { Total } \\
\text { Electricity } \\
\text { Cost }\end{array}$ & PAR & $\begin{array}{c}\text { Total } \\
\text { Electricity } \\
\text { Cost }\end{array}$ & PAR & $\begin{array}{c}\text { Total } \\
\text { Electricity } \\
\text { Cost }\end{array}$ & PAR \\
\hline 1 & $\$ 15,598.71$ & 1.29 & $\$ 16,512.94$ & 1.30 & $\$ 17,531.51$ & 1.31 \\
\hline 2 & $\$ 15,586.61$ & 1.30 & $\$ 16,568.02$ & 1.30 & $\$ 17,491.11$ & 1.31 \\
\hline 3 & $\$ 15,606.09$ & 1.31 & $\$ 16,517.42$ & 1.31 & $\$ 17,501.20$ & 1.31 \\
\hline 4 & $\$ 15,602.24$ & 1.32 & $\$ 16,535.00$ & 1.30 & $\$ 17,476.78$ & 1.31 \\
\hline 5 & $\$ 15,577.76$ & 1.29 & $\$ 16,563.21$ & 1.30 & $\$ 17,506.17$ & 1.31 \\
\hline AVERAGE & $\$ 15,594.28$ & 1.30 & $\$ 16,539.32$ & 1.30 & $\$ 17,501.35$ & 1.31 \\
\hline
\end{tabular}


Table 17: Results for night charging model under linear cost (200 users)

\begin{tabular}{|c|c|c|c|c|c|c|}
\hline \multirow{3}{*}{ Instance } & \multicolumn{3}{|c|}{ Penetration rate of medium-distance users } \\
\cline { 2 - 7 } & \multicolumn{2}{|c|}{$\mathbf{2 0 \%}$} & \multicolumn{2}{c|}{$\mathbf{3 5 \%}$} & \multicolumn{2}{|c|}{$\mathbf{5 0 \%}$} \\
\cline { 2 - 7 } & $\begin{array}{c}\text { Total } \\
\text { Electricity } \\
\text { Cost }\end{array}$ & PAR & $\begin{array}{c}\text { Total } \\
\text { Electricity } \\
\text { Cost }\end{array}$ & PAR & $\begin{array}{c}\text { Total } \\
\text { Electricity } \\
\text { Cost }\end{array}$ & PAR \\
\hline 1 & $\$ 61,541.58$ & 1.28 & $\$ 65,380.36$ & 1.30 & $\$ 69,204.06$ & 1.31 \\
\hline 2 & $\$ 61,597.77$ & 1.29 & $\$ 65,327.83$ & 1.30 & $\$ 69,153.25$ & 1.31 \\
\hline 3 & $\$ 61,625.35$ & 1.28 & $\$ 65,355.92$ & 1.29 & $\$ 69,262.11$ & 1.32 \\
\hline 4 & $\$ 61,565.49$ & 1.29 & $\$ 65,288.76$ & 1.30 & $\$ 69,213.81$ & 1.31 \\
\hline 5 & $\$ 61,646.57$ & 1.28 & $\$ 65,356.30$ & 1.30 & $\$ 69,168.49$ & 1.31 \\
\hline AVERAGE & $\$ 61,595.35$ & 1.28 & $\$ 65,341.83$ & 1.30 & $\$ 69,200.34$ & 1.31 \\
\hline
\end{tabular}

Furthermore, we compare the results for the optimal night-only charging scheduling (Tables 14-17) with the uncontrolled charging (Tables 6-9). In this comparison, we observe improvements both in total cost and PAR as a result of optimal scheduling. For example, at $20 \%$ penetration, there is a $19.13 \%$ reduction in total electricity cost for 10 users. Additionally, the PAR is reduced from 2.32 to 1.39 (40.01\% reduction) under the optimization model.

Table 18 shows the improvements in total cost and PAR for 10, 50, 100 and 200 users for various penetration rates. From Table 18, we see that at $20 \%$ penetration of medium-distance users, there is a $19.13 \%$ improvement in total cost of 10 users and the improvement increases to $22.26 \%$ for a total of 200 users. Similarly, there is an increase in PAR when we implement the optimization model from $40.01 \%$ for 10 users to $44.65 \%$ for 200 users. Thus, we conclude that as the number of users increases from 10 to 200 , the percentages of reduction in cost and PAR both increase. 
On the other hand, in Table 18, when the number of users is fixed, for example $n=50$, the percentages of reduction in cost and PAR are $21.70 \%$ and $43.36 \%$ for penetration rate of $20 \%, 19.82 \%$ and $42.29 \%$ for the penetration rate of $35 \%$, and $17.61 \%$ and $39.66 \%$ for the penetration rate of $55 \%$. Therefore, as the penetration rate of medium-distance users increases from $20 \%$ to $35 \%$ to $50 \%$, the percentages of reduction in both cost and PAR decrease.

Table 18: Optimal night charging versus uncontrolled charging (linear cost)

\begin{tabular}{|c|c|c|c|c|c|c|c|c|}
\hline \multirow{2}{*}{$\begin{array}{c}\text { Penetration } \\
\text { rate of } \\
\text { medium- } \\
\text { distance } \\
\text { users }\end{array}$} & \multicolumn{2}{|c|}{$\mathbf{n = 1 0}$} & \multicolumn{2}{c|}{$\mathbf{n = 5 0}$} & \multicolumn{2}{c|}{$\mathbf{n = 1 0 0}$} & \multicolumn{2}{c|}{$\mathbf{n = 2 0 0}$} \\
\cline { 2 - 9 } & $\begin{array}{c}\text { Cost } \\
\text { reduced }\end{array}$ & $\begin{array}{c}\text { PAR } \\
\text { reduced } \\
(\%)\end{array}$ & $\begin{array}{c}\text { Cost } \\
\text { reduced } \\
(\%)\end{array}$ & $\begin{array}{c}\text { PAR } \\
\text { reduced } \\
(\%)\end{array}$ & $\begin{array}{c}\text { Cost } \\
\text { reduced } \\
(\%)\end{array}$ & $\begin{array}{c}\text { PAR } \\
\text { reduced } \\
(\%)\end{array}$ & $\begin{array}{c}\text { Cost } \\
\text { reduced } \\
(\%)\end{array}$ & $\begin{array}{c}\text { PAR } \\
\text { reduce } \\
\mathbf{d}(\%)\end{array}$ \\
\hline $20 \%$ & 19.13 & 40.01 & 21.70 & 43.36 & 22.12 & 43.76 & 22.26 & 44.65 \\
\hline $35 \%$ & 17.76 & 39.60 & 19.82 & 42.29 & 19.86 & 42.47 & 19.99 & 42.58 \\
\hline $50 \%$ & 15.99 & 37.81 & 17.61 & 39.66 & 17.99 & 40.58 & 18.28 & 40.62 \\
\hline
\end{tabular}

Figure 5 shows the improvement in load leveling by the optimal night charging scheduling model. The residential load profile shown in Figure 5 is generated based on the results for 10 users but similar load profiles are also seen for 50, 100 and 500 users. From Figure 5, we observe that the peak demand between $6: 00 \mathrm{pm}$ to midnight is significantly reduced. Furthermore, we notice an increase in energy consumption between midnight to 7:00 am. Hence, the charging scheduling of our model is efficient in load leveling by shifting the load during peak hours to fill in the "valley" between midnight to 7:00 am. 


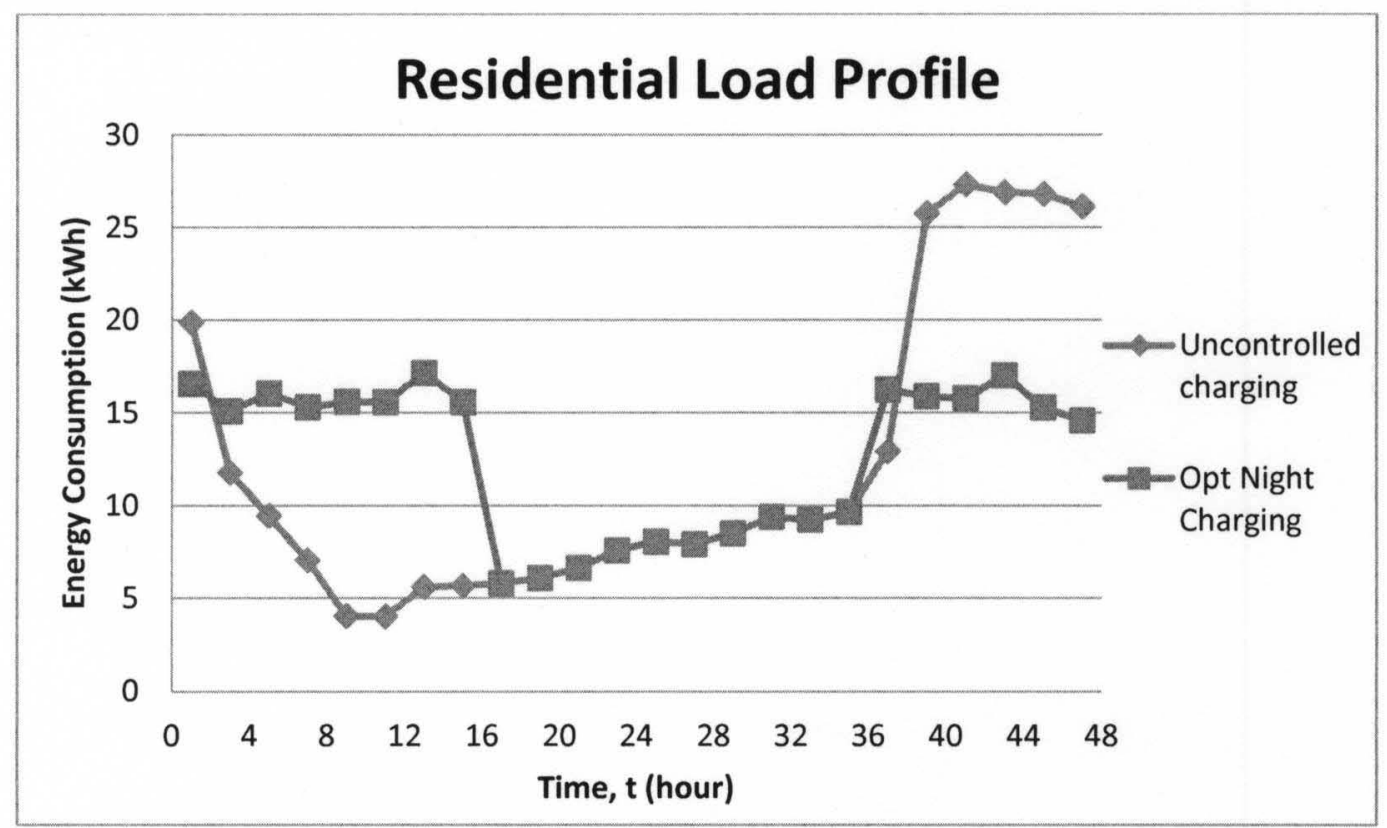

Figure 5: Load leveling under optimal night charging model

Figures 6 and 7 display the charging profiles for a medium-distance BEV user (User 1) under the uncontrolled and night-only optimal charging scenarios, respectively. From Figure 6, we notice that User 1 starts charging at 7:00 pm $(t=39)$ and completes his/her charging by 4:00 am the following day. Further, we note that the charging is done continuously based on the current algorithm for charging in EVs. Figure 7 shows the charging scheduling for the User 1 under the centralized optimal night charging model. We see that there are several separate and disjoint charging intervals for User 1 . The allocation given by the central controller is based on the price at the specific charging time interval such that the user's total electricity cost is minimized. 


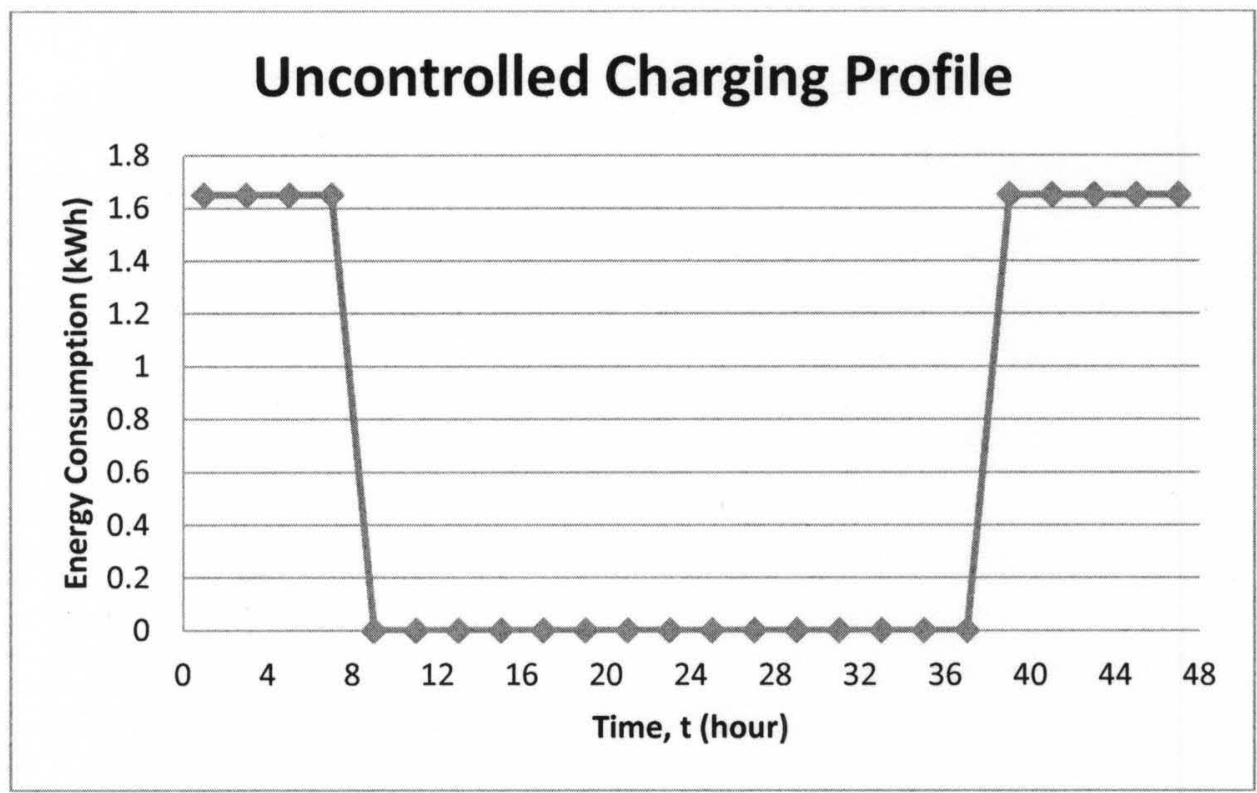

Figure 6: Uncontrolled charging profile for User 1

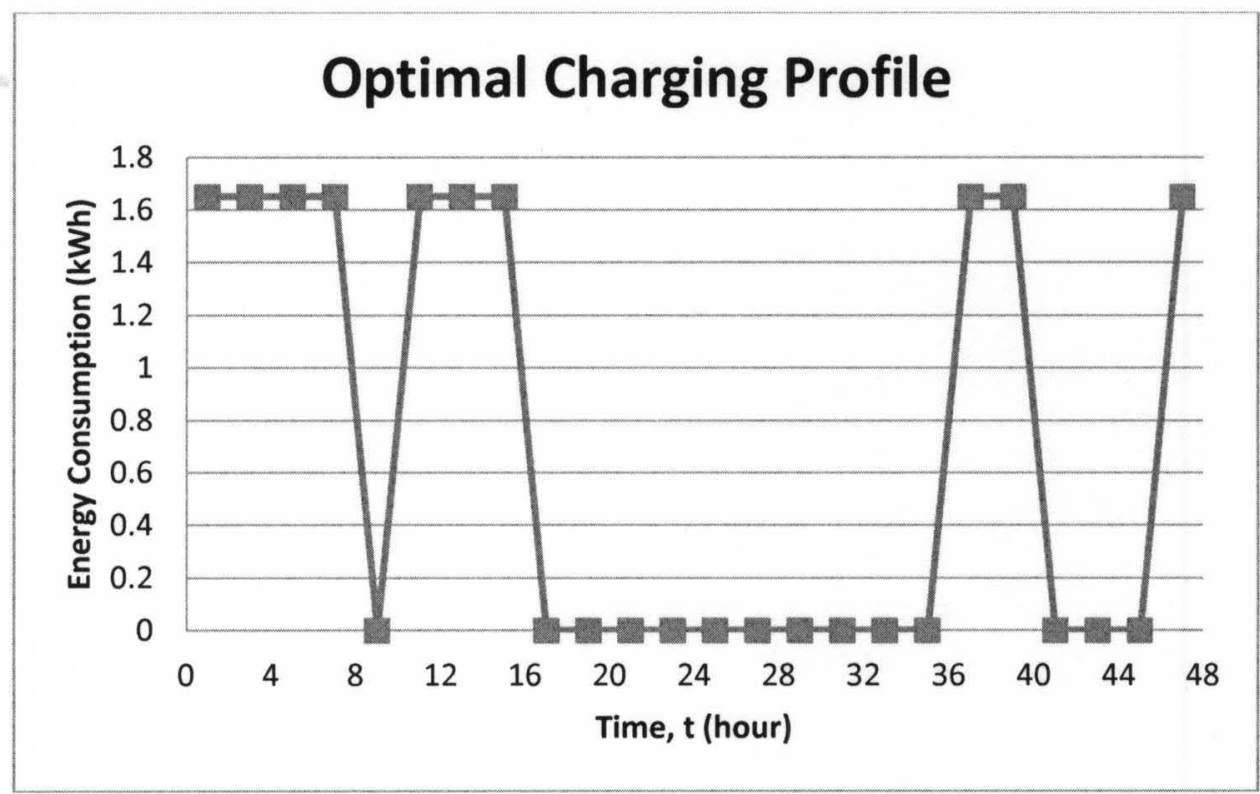

Figure 7: Optimal nighttime charging schedule for User 1 


\subsubsection{Results for optimal charging with day-and-night charging}

The results presented in this section are from the optimal charging scheduling when charging is allowed during the day and night.

Table 19: Results for day-and-night charging model under linear cost (10 users)

\begin{tabular}{|c|c|c|c|c|c|c|}
\hline \multirow{3}{*}{ Instance } & \multicolumn{3}{|c|}{ Penetration rate of medium-distance users } \\
\cline { 2 - 7 } & \multicolumn{2}{|c|}{$\mathbf{2 0 \%}$} & \multicolumn{2}{c|}{$\mathbf{3 5 \%}$} & \multicolumn{2}{c|}{$\mathbf{5 0 \%}$} \\
\cline { 2 - 7 } & $\begin{array}{c}\text { Total } \\
\text { Electricity } \\
\text { Cost }\end{array}$ & PAR & $\begin{array}{c}\text { Total } \\
\text { Electricity } \\
\text { Cost }\end{array}$ & PAR & $\begin{array}{c}\text { Total } \\
\text { Electricity } \\
\text { Cost }\end{array}$ & PAR \\
\hline 1 & $\$ 180.25$ & 1.12 & $\$ 186.22$ & 1.18 & $\$ 215.68$ & 1.15 \\
\hline 2 & $\$ 180.87$ & 1.16 & $\$ 186.23$ & 1.19 & $\$ 214.90$ & 1.14 \\
\hline 3 & $\$ 180.78$ & 1.12 & $\$ 186.87$ & 1.16 & $\$ 215.90$ & 1.14 \\
\hline 4 & $\$ 181.19$ & 1.11 & $\$ 185.98$ & 1.13 & $\$ 215.61$ & 1.20 \\
\hline 5 & $\$ 182.25$ & 1.38 & $\$ 185.51$ & 1.11 & $\$ 215.59$ & 1.15 \\
\hline AVERAGE & $\$ 181.07$ & 1.18 & $\$ 186.16$ & 1.15 & $\$ 215.53$ & 1.16 \\
\hline
\end{tabular}

Table 19 shows the results for day-and-night charging model for 10 users. From the results, we observe that the average total electricity cost is $\$ 181.07$ at $20 \%$ penetration and increases to $\$ 215.53$ at $50 \%$ penetration. On the other hand, the PAR decreases from 1.18 at $20 \%$ penetration to 1.15 at $35 \%$ penetration. The average PAR for $50 \%$ then increases slightly to 1.16 . This indicates that PAR is not significantly affected by the penetration rate of medium distance users. This is consistent with the observation from Table 14 for the optimal night-only charging scenario. Similar to Table 19, Tables 20,21 and 22 display the results for 50,100 and 200 users, respectively. They also suggest that penetration rate has a positive impact on the cost and little impact on the PAR. 
Table 20: Results for day-and-night charging model under linear cost (50 users)

\begin{tabular}{|c|c|c|c|c|c|c|}
\hline \multirow{3}{*}{ Instance } & \multicolumn{3}{|c|}{ Penetration rate of medium-distance users } \\
\cline { 2 - 7 } & \multicolumn{2}{|c|}{$\mathbf{2 0 \%}$} & \multicolumn{2}{c|}{$\mathbf{3 5 \%}$} & \multicolumn{2}{c|}{$\mathbf{5 0 \%}$} \\
\cline { 2 - 7 } & $\begin{array}{c}\text { Total } \\
\text { Electricity } \\
\text { Cost }\end{array}$ & PAR & $\begin{array}{c}\text { Total } \\
\text { Electricity } \\
\text { Cost }\end{array}$ & PAR & $\begin{array}{c}\text { Total } \\
\text { Electricity } \\
\text { Cost }\end{array}$ & PAR \\
\hline 1 & $\$ 3,631.78$ & 1.12 & $\$ 3,824.64$ & 1.06 & $\$ 4,039.22$ & 1.03 \\
\hline 2 & $\$ 3,652.21$ & 1.06 & $\$ 3,812.50$ & 1.04 & $\$ 4,011.07$ & 1.03 \\
\hline 3 & $\$ 3,639.01$ & 1.05 & $\$ 3,819.23$ & 1.04 & $\$ 4,034.66$ & 1.04 \\
\hline 4 & $\$ 3,647.82$ & 1.06 & $\$ 3,820.82$ & 1.04 & $\$ 4,022.43$ & 1.02 \\
\hline 5 & $\$ 3,651.26$ & 1.06 & $\$ 3,807.30$ & 1.03 & $\$ 4,034.02$ & 1.03 \\
\hline AVERAGE & $\$ 3,644.41$ & 1.07 & $\$ 3,816.90$ & 1.04 & $\$ 4,028.28$ & 1.03 \\
\hline
\end{tabular}

Table 21: Results for day-and-night charging model under linear cost (100 users)

\begin{tabular}{|c|c|c|c|c|c|c|}
\hline \multirow{3}{*}{ Instance } & \multicolumn{6}{|c|}{ Penetration rate of medium-distance users } \\
\cline { 2 - 7 } & \multicolumn{2}{|c|}{$\mathbf{2 0 \%}$} & \multicolumn{2}{c|}{$\mathbf{3 5 \%}$} & \multicolumn{2}{c|}{$\mathbf{5 0 \%}$} \\
\cline { 2 - 7 } & $\begin{array}{c}\text { Total } \\
\text { Electricity } \\
\text { Cost }\end{array}$ & PAR & $\begin{array}{c}\text { Total } \\
\text { Electricity } \\
\text { Cost }\end{array}$ & PAR & $\begin{array}{c}\text { Total } \\
\text { Electricity } \\
\text { Cost }\end{array}$ & PAR \\
\hline 1 & $\$ 14,154.42$ & 1.02 & $\$ 14,853.47$ & 1.01 & $\$ 15,661.53$ & 1.02 \\
\hline 2 & $\$ 14,135.35$ & 1.02 & $\$ 14,908.73$ & 1.02 & $\$ 15,618.27$ & 1.02 \\
\hline 3 & $\$ 14,153.64$ & 1.02 & $\$ 14,857.63$ & 1.02 & $\$ 15,625.83$ & 1.02 \\
\hline 4 & $\$ 14,150.16$ & 1.02 & $\$ 14,879.57$ & 1.02 & $\$ 15,602.59$ & 1.02 \\
\hline 5 & $\$ 14,127.84$ & 1.01 & $\$ 14,909.92$ & 1.01 & $\$ 15,631.65$ & 1.02 \\
\hline AVERAGE & $\$ 14,144.28$ & 1.02 & $\$ 14,881.86$ & 1.02 & $\$ 15,627.97$ & 1.02 \\
\hline
\end{tabular}

Table 22: Results for day-and-night charging model under linear cost (200 users)

\begin{tabular}{|c|c|c|c|c|c|c|}
\hline \multirow{3}{*}{ Instance } & \multicolumn{6}{|c|}{ Penetration rate of medium-distance users } \\
\cline { 2 - 7 } & \multicolumn{2}{|c|}{$\mathbf{2 0 \%}$} & \multicolumn{2}{c|}{$\mathbf{3 5 \%}$} & \multicolumn{2}{c|}{$\mathbf{5 0 \%}$} \\
\cline { 2 - 7 } & $\begin{array}{c}\text { Total } \\
\text { Electricity } \\
\text { Cost }\end{array}$ & PAR & $\begin{array}{c}\text { Total } \\
\text { Electricity } \\
\text { Cost }\end{array}$ & PAR & $\begin{array}{c}\text { Total } \\
\text { Electricity } \\
\text { Cost }\end{array}$ & PAR \\
\hline 1 & $\$ 55,737.33$ & 1.01 & $\$ 58,728.26$ & 1.01 & $\$ 61,666.61$ & 1.01 \\
\hline 2 & $\$ 55,785.29$ & 1.01 & $\$ 58,706.18$ & 1.01 & $\$ 61,679.88$ & 1.01 \\
\hline 3 & $\$ 55,833.91$ & 1.01 & $\$ 58,768.47$ & 1.01 & $\$ 61,743.69$ & 1.01 \\
\hline 4 & $\$ 55,778.74$ & 1.01 & $\$ 58,673.87$ & 1.01 & $\$ 61,699.17$ & 1.01 \\
\hline 5 & $\$ 55,858.48$ & 1.01 & $\$ 58,739.93$ & 1.01 & $\$ 61,643.97$ & 1.01 \\
\hline AVERAGE & $\$ 55,798.75$ & 1.01 & $\$ 58,723.34$ & 1.01 & $\$ 61,686.66$ & 1.01 \\
\hline
\end{tabular}


Table 23: Optimal day-and-night versus uncontrolled charging (linear cost)

\begin{tabular}{|c|c|c|c|c|c|c|c|c|}
\hline \multirow{2}{*}{$\begin{array}{c}\text { Penetration } \\
\text { rate of } \\
\text { medium- } \\
\text { distance } \\
\text { users }\end{array}$} & \multicolumn{2}{|c|}{$\mathbf{n = 1 0}$} & \multicolumn{2}{c|}{$\mathbf{n = 5 0}$} & \multicolumn{2}{c|}{$\mathbf{n = 1 0 0}$} & \multicolumn{2}{c|}{$\mathbf{n = 2 0 0}$} \\
\cline { 2 - 9 } & $\begin{array}{c}\text { Cost } \\
\text { reduced }\end{array}$ & $\begin{array}{c}\text { PAR } \\
\text { reduced } \\
(\%)\end{array}$ & $\begin{array}{c}\text { Cost } \\
\text { reduced } \\
(\%)\end{array}$ & $\begin{array}{c}\text { PAR } \\
\text { reduced } \\
(\%)\end{array}$ & $\begin{array}{c}\text { Cost } \\
\text { reduced } \\
(\%)\end{array}$ & $\begin{array}{c}\text { PAR } \\
\text { reduced } \\
(\%)\end{array}$ & $\begin{array}{c}\text { Cost } \\
\text { reduced } \\
(\%)\end{array}$ & $\begin{array}{c}\text { PAR } \\
\text { reduced } \\
(\%)\end{array}$ \\
\hline $20 \%$ & 24.91 & 49.33 & 28.78 & 53.98 & 29.36 & 55.97 & 29.58 & 56.50 \\
\hline $35 \%$ & 24.07 & 49.34 & 27.61 & 54.02 & 27.90 & 54.98 & 28.10 & 55.27 \\
\hline $50 \%$ & 23.14 & 47.80 & 26.18 & 53.17 & 26.77 & 53.75 & 27.15 & 54.30 \\
\hline
\end{tabular}

We also compare the results of uncontrolled charging (Tables 6-9) with the solutions from the optimal day-and-night charging model (Tables 19-22), and summarize the comparison in Table 23. From Table 23, we see an increasing cost reduction overall as we increase the number of users from 10 to 200 . For example, the total cost reduction at $20 \%$ penetration is $24.91 \%$ for 10 users and the cost reduction increases to $29.58 \%$ for 200 users. We notice a similar trend for the percentage of PAR reduction. This indicates that the optimal scheduling model becomes more beneficial as more users are included. On the other hand, penetration rate does not have much effect on the reduction of cost or PAR, when the number of users is fixed. This is consistent with the comparison shown in Table 18 for the optimal night-only charging model.

In addition, Figure 8 compares the aggregated load profiles for 10 users under the uncontrolled and optimal day-and-night charging scenarios, respectively. From Figure 8, we observe that a more leveled load distribution is achieved throughout the 48 half-hour cycles by the optimization scheduling. Further, the initial high peak demand occurring between 6:00 pm to midnight is substantially reduced by approximately $50 \%$. 


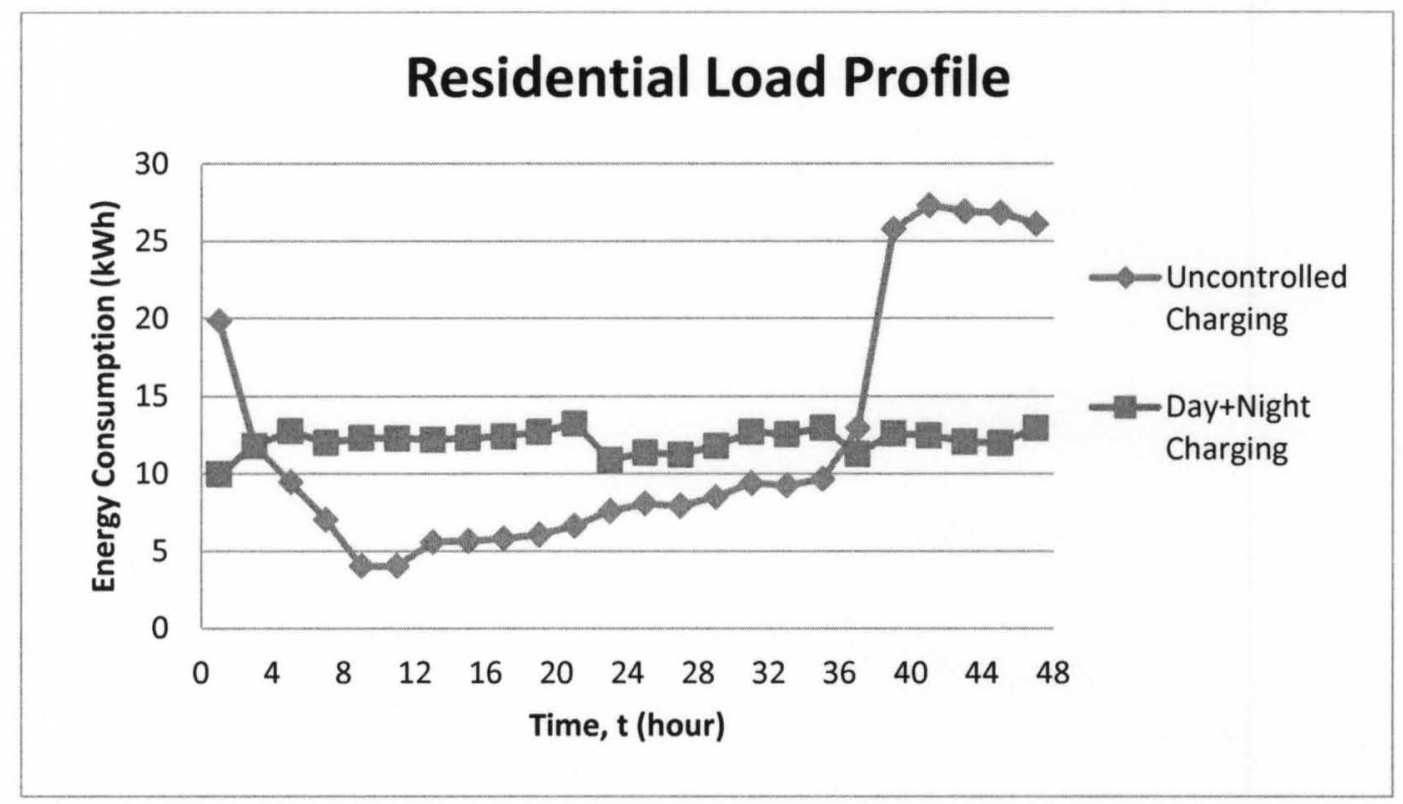

Figure 8: Load leveling under optimal day-and-night charging model

Figure 9 illustrates the optimal charging profile for the medium-distance BEV User 1 under the day-and-night charging scenario. Notice that for the same User 1, we have illustrated his/her uncontrolled and optimal night-only charging profiles in Figures 6 and 7, respectively. From Figure 9, we see that a total charge of 3 hours during the day between 8:00 am to 5:30 pm is assigned to this user. For each charging during the day, the user is enforced to charge for two consecutive time intervals, i.e., 1 hour for his/her convenience. Nonetheless, the charging schedule during the evening hours is not restricted to the 1-hour charging constraint. In Figure 9, we observe that there is some time intervals $t$ where the User 1 is scheduled to charge for only one time interval $t$ at 11:30 pm, and other intervals where User 1 is assigned to charge for two consecutive intervals, i.e., $12: 00 \mathrm{am}$ to $1: 00 \mathrm{am}$. 


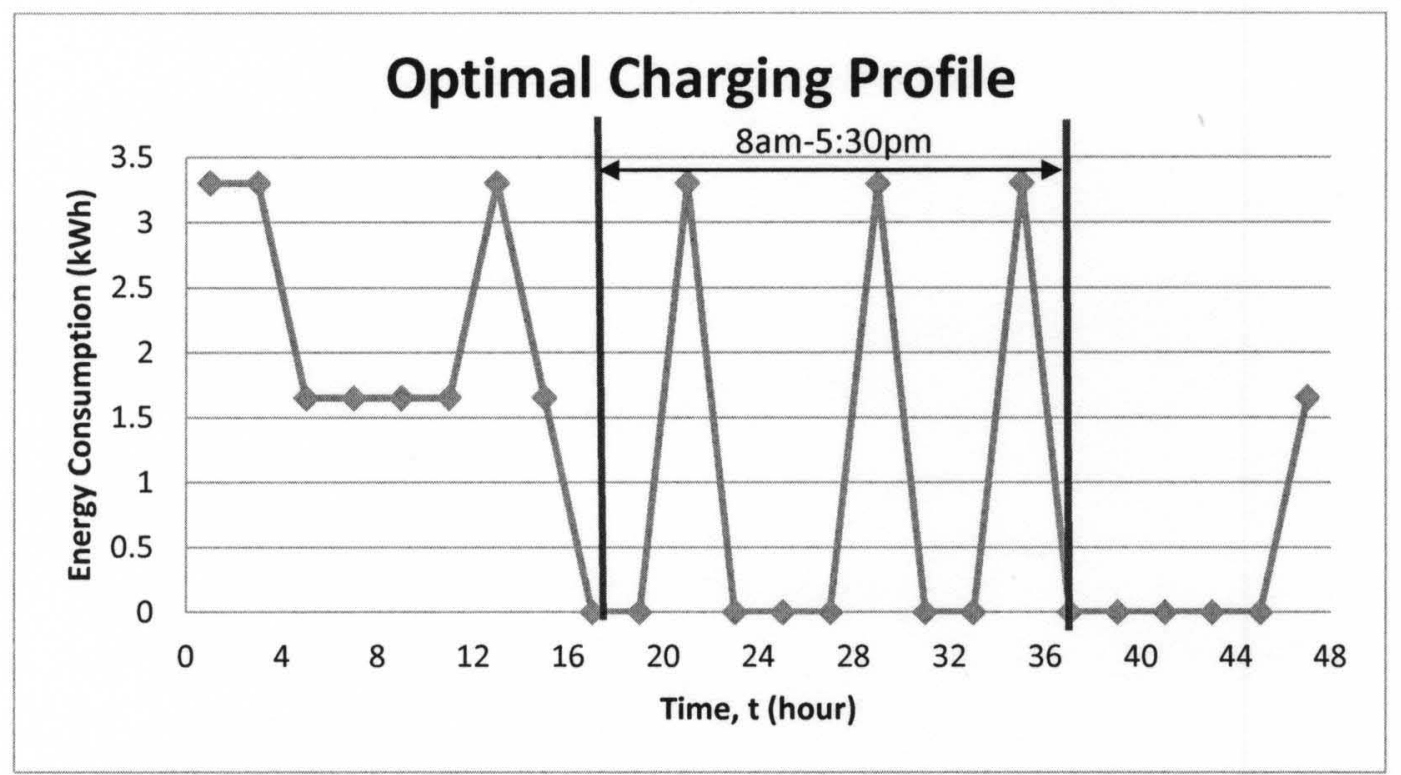

Figure 9: Optimal day-and-night charging schedule for User 1

\subsubsection{Comparisons between results for night-only vs. day-and-night charging}

In comparing the night-only and day-and-night charging scenarios for the centralized optimization model, we see that the percent reduction in PAR for 10 users is approximately $40 \%$ under a night-only charging model compared to the uncontrolled charging. However, an even higher percent reduction in PAR (49\%) is achieved under the optimal day-and-night charging model. As we increase the number of users, we notice that the optimal day-and-night charging model continues to provide a higher reduction in PAR than does the night-only charging scenario. For example, the numerical results for 200 users showed a 55\% improvement in PAR under the day-and-night charging model, while the PAR is improved by $44 \%$ under the night-only charging model. The reason for a higher PAR reduction in the former is due to a larger interval for the central controller to schedule EV charging for users. Hence, the energy demand can be more evenly 
distributed. Figure 10 illustrates a comparison of load leveling achieved through the two charging scenarios under a centralized approach for 10 users. Similar load profiles are seen across different numbers of users, i.e., 50, 100 and 200.

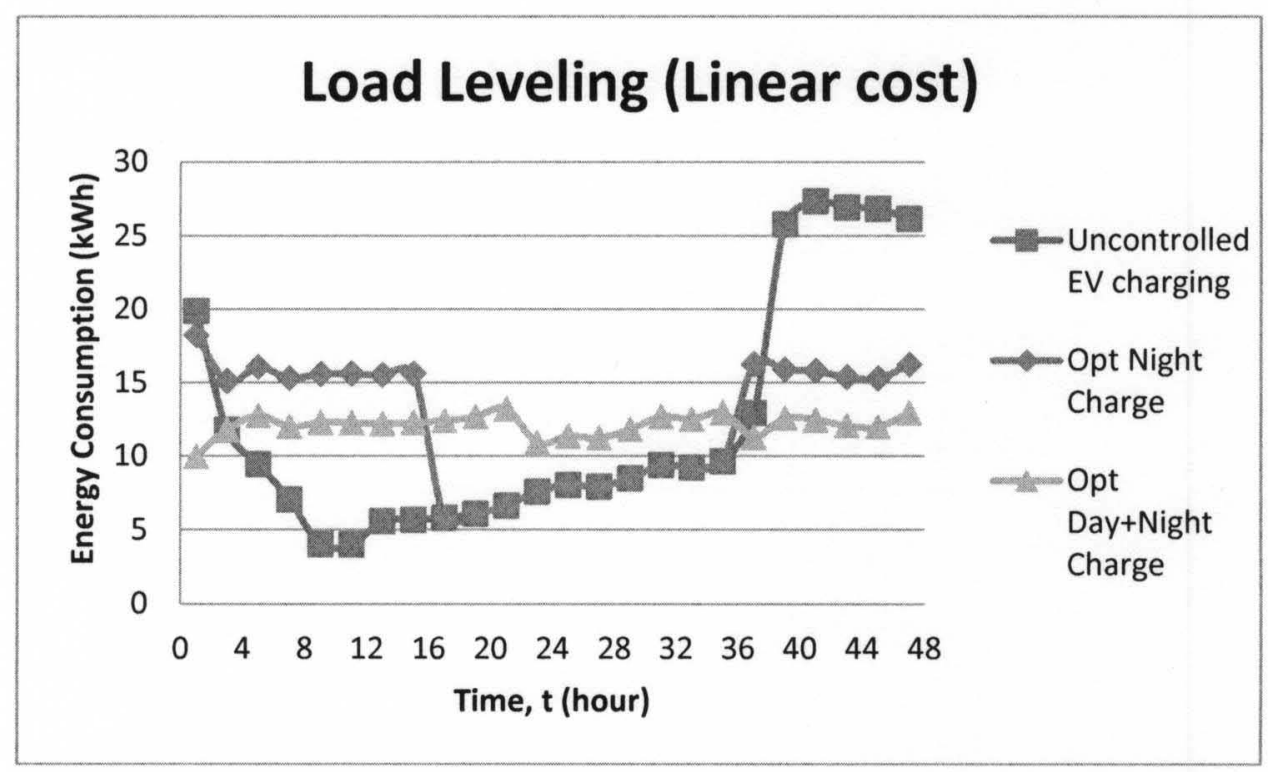

Figure 10: Overall load leveling under linear cost function

From Figure 10, we see that the load profile under the day-and-night scenario contains no significant peaks, i.e., the load profile is flattened across the entire time intervals. Hence, we deduce that allowing user to charge at work is advantageous in reducing the system's overall PAR. Reducing peak demand loads, especially during the evening hours, indirectly implies that the dependency on expensive sources of electricity generators by power plants can be lowered.

We also observe that the improvement in total electricity cost incurred to all users under the day-and-night charging model is slightly better compared to the night-only charging model. The total cost reduction for 200 users is approximately $21 \%$ under the 
night-only charging model. On the other hand, the cost is reduced by approximately $29 \%$ under the day-and-night charging model. The difference of the total cost reduction between these two charging scenarios is caused by the extra flexibility offered in the dayand-night charging scenario. In particular, the day-and-night charging scenario has more flexibility in scheduling the EV charging between 8:00 am to 5:30 pm where the baseline household demand is low. Hence, the user would minimize his/her total electricity cost by shifting the load from EV charging to the periods of low energy consumption.

\subsubsection{Results under time-of-use pricing structure}

\subsubsection{Results for optimal charging with night charging only}

Table 24 displays the performance measurers for night-only charging scenario under time-of-use pricing structure for 10 users. From Table 24, we observe that the total cost of 10 users increase from $\$ 35.22$ at $20 \%$ penetration rate to $\$ 35.65$ when the penetration rate is increased to $35 \%$. Further, at $50 \%$, the total electricity cost is calculated to be $\$ 36.62$. The PAR value, on the other hand, decreases as we increase the penetration rate. The PAR decreases from 2.18 at $20 \%$ penetration to 2.08 at $50 \%$ penetration. Tables 25,26 and 27 show the results for night-only charging scenario for 50, 100 and 200 users, respectively; and they exhibit similar trend in total cost and PAR as the penetration rate increases. 
Table 24: Results for night-only charging under TOU (10 users)

\begin{tabular}{|c|c|c|c|c|c|c|}
\hline \multirow{3}{*}{ Instance } & \multicolumn{3}{|c|}{ Penetration rate of medium-distance users } \\
\cline { 2 - 7 } & \multicolumn{2}{|c|}{$\mathbf{2 0 \%}$} & \multicolumn{2}{c|}{$\mathbf{3 5 \%}$} & \multicolumn{2}{c|}{$\mathbf{5 0 \%}$} \\
\cline { 2 - 7 } & $\begin{array}{c}\text { Total } \\
\text { Electricity } \\
\text { Cost }\end{array}$ & PAR & $\begin{array}{c}\text { Total } \\
\text { Electricity } \\
\text { Cost }\end{array}$ & PAR & $\begin{array}{c}\text { Total } \\
\text { Electricity } \\
\text { Cost }\end{array}$ & PAR \\
\hline 1 & $\$ 35.12$ & 2.18 & $\$ 35.70$ & 2.14 & $\$ 36.70$ & 2.05 \\
\hline 2 & $\$ 35.21$ & 2.17 & $\$ 35.66$ & 2.15 & $\$ 36.53$ & 2.10 \\
\hline 3 & $\$ 35.19$ & 2.18 & $\$ 35.73$ & 2.16 & $\$ 36.65$ & 2.07 \\
\hline 4 & $\$ 35.26$ & 2.19 & $\$ 35.63$ & 2.17 & $\$ 36.66$ & 2.08 \\
\hline 5 & $\$ 35.33$ & 2.21 & $\$ 35.55$ & 2.14 & $\$ 36.58$ & 2.08 \\
\hline AVERAGE & $\$ 35.22$ & 2.18 & $\$ 35.65$ & 2.15 & $\$ 36.62$ & 2.08 \\
\hline
\end{tabular}

Table 25: Results for night-only charging under TOU (50 users)

\begin{tabular}{|c|c|c|c|c|c|c|}
\hline \multirow[b]{3}{*}{ Instance } & \multicolumn{6}{|c|}{ Penetration rate of medium-distance users } \\
\hline & \multicolumn{2}{|c|}{$20 \%$} & \multicolumn{2}{|c|}{$35 \%$} & \multicolumn{2}{|c|}{$50 \%$} \\
\hline & $\begin{array}{c}\text { Total } \\
\text { Electricity } \\
\text { Cost }\end{array}$ & PAR & $\begin{array}{c}\text { Total } \\
\text { Electricity } \\
\text { Cost }\end{array}$ & PAR & $\begin{array}{c}\text { Total } \\
\text { Electricity } \\
\text { Cost }\end{array}$ & PAR \\
\hline 1 & $\$ 174.29$ & 2.18 & $\$ 178.45$ & 2.13 & $\$ 182.76$ & 2.09 \\
\hline 2 & $\$ 174.98$ & 2.19 & $\$ 178.08$ & 2.13 & $\$ 181.94$ & 2.07 \\
\hline 3 & $\$ 174.57$ & 2.18 & $\$ 178.20$ & 2.13 & $\$ 182.63$ & 2.08 \\
\hline 4 & $\$ 174.77$ & 2.17 & $\$ 178.27$ & 2.13 & $\$ 182.24$ & 2.08 \\
\hline 5 & $\$ 174.81$ & 2.19 & $\$ 177.89$ & 2.14 & $\$ 182.71$ & 2.06 \\
\hline AVERAGE & $\$ 174.68$ & 2.18 & $\$ 178.18$ & 2.13 & $\$ 182.46$ & 2.08 \\
\hline
\end{tabular}

Table 26: Results for night-only charging under TOU (100 users)

\begin{tabular}{|c|c|c|c|c|c|c|}
\hline \multirow{3}{*}{ Instance } & \multicolumn{6}{|c|}{ Penetration rate of medium-distance users } \\
\cline { 2 - 7 } & \multicolumn{2}{|c|}{$\mathbf{2 0 \%}$} & \multicolumn{2}{c|}{$\mathbf{3 5 \%}$} & \multicolumn{2}{c|}{$\mathbf{5 0 \%}$} \\
\cline { 2 - 7 } & $\begin{array}{c}\text { Total } \\
\text { Electricity } \\
\text { Cost }\end{array}$ & PAR & $\begin{array}{c}\text { Total } \\
\text { Electricity } \\
\text { Cost }\end{array}$ & PAR & $\begin{array}{c}\text { Total } \\
\text { Electricity } \\
\text { Cost }\end{array}$ & PAR \\
\hline 1 & $\$ 349.14$ & 2.17 & $\$ 356.25$ & 2.12 & $\$ 364.55$ & 2.07 \\
\hline 2 & $\$ 348.88$ & 2.17 & $\$ 356.96$ & 2.13 & $\$ 364.02$ & 2.06 \\
\hline 3 & $\$ 349.09$ & 2.18 & $\$ 356.23$ & 2.13 & $\$ 364.04$ & 2.08 \\
\hline 4 & $\$ 349.03$ & 2.19 & $\$ 356.64$ & 2.12 & $\$ 363.74$ & 2.07 \\
\hline 5 & $\$ 348.85$ & 2.17 & $\$ 357.03$ & 2.12 & $\$ 364.21$ & 2.07 \\
\hline AVERAGE & $\$ 349.00$ & 2.18 & $\$ 356.62$ & 2.13 & $\$ 364.11$ & 2.07 \\
\hline
\end{tabular}


Table 27: Results for night-only charging under TOU (200 users)

\begin{tabular}{|c|c|c|c|c|c|c|}
\hline \multirow{4}{*}{ Instance } & \multicolumn{5}{|c|}{ Penetration rate of medium-distance users } \\
\cline { 2 - 7 } & \multicolumn{2}{|c|}{$\mathbf{2 0 \%}$} & \multicolumn{2}{|c|}{$\mathbf{3 5 \%}$} & \multicolumn{2}{|c|}{$\mathbf{5 0 \%}$} \\
\cline { 2 - 7 } & $\begin{array}{c}\text { Total } \\
\text { Electricity } \\
\text { Cost }\end{array}$ & PAR & $\begin{array}{c}\text { Total } \\
\text { Electricity } \\
\text { Cost }\end{array}$ & PAR & $\begin{array}{c}\text { Total } \\
\text { Electricity } \\
\text { Cost }\end{array}$ & PAR \\
\hline 1 & $\$ 697.89$ & 2.17 & $\$ 713.56$ & 2.13 & $\$ 728.20$ & 2.08 \\
\hline 2 & $\$ 698.23$ & 2.18 & $\$ 713.38$ & 2.12 & $\$ 728.60$ & 2.07 \\
\hline 3 & $\$ 698.59$ & 2.18 & $\$ 713.94$ & 2.12 & $\$ 728.88$ & 2.07 \\
\hline 4 & $\$ 698.18$ & 2.18 & $\$ 713.21$ & 2.12 & $\$ 728.64$ & 2.07 \\
\hline 5 & $\$ 698.71$ & 2.18 & $\$ 713.68$ & 2.13 & $\$ 728.09$ & 2.08 \\
\hline AVERAGE & $\$ 698.32$ & 2.18 & $\$ 713.56$ & 2.13 & $\$ 728.48$ & 2.07 \\
\hline
\end{tabular}

Table 28: Optimal night-only model versus uncontrolled charging under TOU

\begin{tabular}{|c|c|c|c|c|c|c|c|c|}
\hline \multirow{2}{*}{$\begin{array}{c}\text { Penetration } \\
\text { rate of } \\
\text { medium- } \\
\text { distance } \\
\text { users }\end{array}$} & \multicolumn{2}{|c|}{$\mathbf{n = 1 0}$} & \multicolumn{2}{c|}{$\mathbf{n = 5 0}$} & \multicolumn{2}{c|}{$\mathbf{n = 1 0 0}$} & \multicolumn{2}{c|}{$\mathbf{n = 2 0 0}$} \\
\cline { 2 - 9 } & $\begin{array}{c}\text { Cost } \\
\text { reduced }\end{array}$ & $\begin{array}{c}\text { PAR } \\
\text { reduced } \\
(\%)\end{array}$ & $\begin{array}{c}\text { Cost } \\
\text { reduced } \\
(\%)\end{array}$ & $\begin{array}{c}\text { PAR } \\
\text { reduced } \\
(\%)\end{array}$ & $\begin{array}{c}\text { Cost } \\
\text { reduced } \\
(\%)\end{array}$ & $\begin{array}{c}\text { PAR } \\
\text { reduced } \\
(\%)\end{array}$ & $\begin{array}{c}\text { Cost } \\
\text { reduced } \\
(\%)\end{array}$ & $\begin{array}{c}\text { PAR } \\
\text { reduced } \\
(\%)\end{array}$ \\
\hline $20 \%$ & 9.23 & 6.09 & 9.37 & 5.93 & 9.84 & 5.85 & 9.78 & 6.13 \\
\hline $35 \%$ & 8.90 & 5.28 & 8.73 & 5.93 & 9.42 & 6.01 & 9.40 & 5.89 \\
\hline $50 \%$ & 8.12 & 6.28 & 7.76 & 5.89 & 9.01 & 6.03 & 9.07 & 6.16 \\
\hline
\end{tabular}

Table 28 summarizes the improvements made by the night-only charging model under the time-of-use pricing structure compared to the uncontrolled EV charging scenario by $10,50,100$ and 200 users, as presented in Tables 10 through 13 . We notice that the reduction in total cost and PAR are similar for all user sizes 10, 50, 100 and 200. Generally, the total cost is improved by approximately $9 \%$ regardless of user size under the time-of-use pricing structure. Likewise, the PAR is reduced by approximately $6 \%$ in general regardless of the user size. 
Figure 11 illustrates the results the optimal night-only charging model for 50 users. From Figure 11, we notice that the demand load from 5:30 pm $(t=36)$ to $11: 00 \mathrm{pm}$ $(t=47)$ is totally shifted to fill in the "valley" between midnight and 7:00 am $(t=13)$. We note that this shift in load is as a result of cheaper price between midnight to early morning as proposed by Collins and Mader (1983). Additionally, we notice a slightly decreased peak (between midnight and 7am) for the optimal night-only charging compared to the peak (between 6pm and midnight) for the uncontrolled charging.

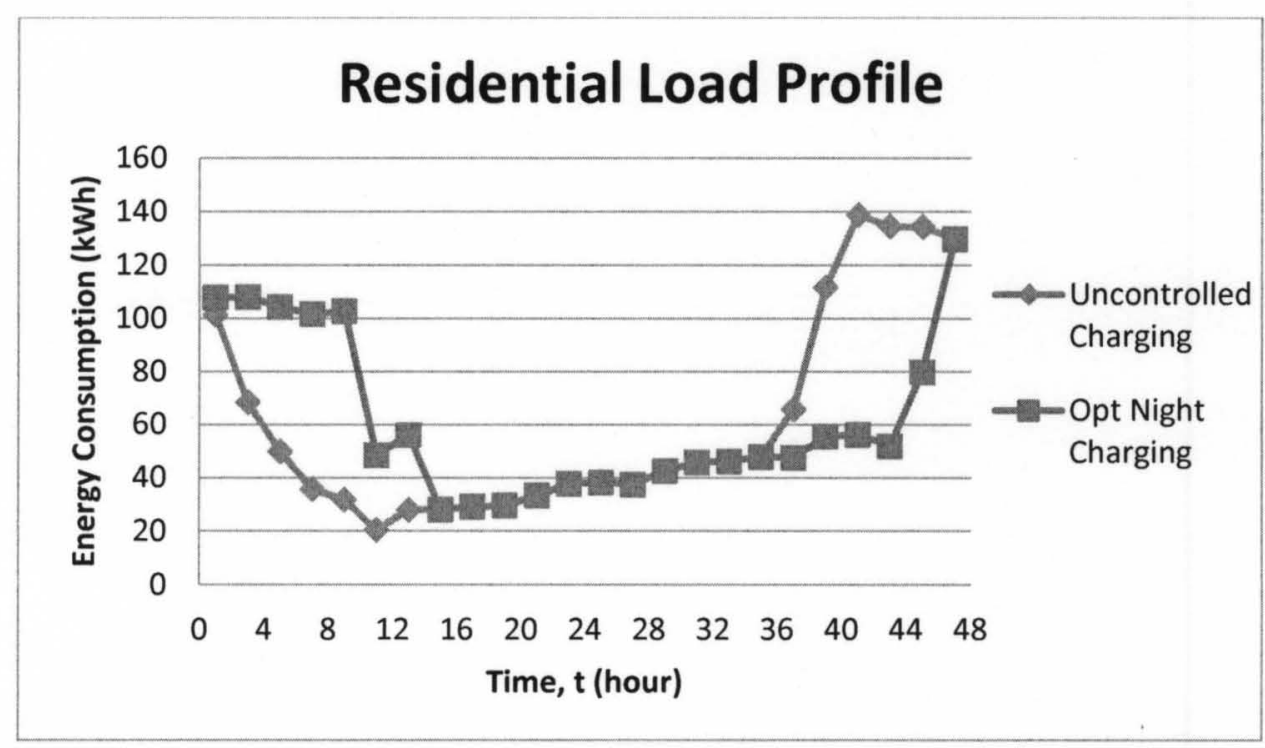

Figure 11: Load leveling under optimal night charging (TOU)

\subsubsection{Results for optimal charging with day-and-night charging}

Tables 29, 30, 31 and 32 display the results for day-and-night charging under time-of-use pricing structure for 10, 50, 100 and 200 users, respectively. In general, the total electricity cost increases as the penetration rate increases. Additionally, the PAR decreases for an increasing penetration rate of medium-distance users. Similar to the results for night-only charging scenario, we observe that the average PAR is almost 
constant at a specific penetration rate regardless of the size of user $n$. For instance, at $20 \%$ penetration rate, the PAR is 2.18 for users $10,50,100$ and 200 .

Table 29: Results for day-and-night charging under TOU (10 users)

\begin{tabular}{|c|c|c|c|c|c|c|}
\hline \multirow{3}{*}{ Instance } & \multicolumn{3}{|c|}{ Penetration rate of medium-distance users } \\
\cline { 2 - 7 } & \multicolumn{2}{|c|}{$\mathbf{2 0 \%}$} & \multicolumn{2}{c|}{$\mathbf{3 5 \%}$} & \multicolumn{2}{c|}{$\mathbf{5 0 \%}$} \\
\cline { 2 - 7 } & $\begin{array}{c}\text { Total } \\
\text { Electricity } \\
\text { Cost }\end{array}$ & PAR & $\begin{array}{c}\text { Total } \\
\text { Electricity } \\
\text { Cost }\end{array}$ & PAR & $\begin{array}{c}\text { Total } \\
\text { Electricity } \\
\text { Cost }\end{array}$ & PAR \\
\hline 1 & $\$ 35.29$ & 2.18 & $\$ 35.95$ & 2.14 & $\$ 37.12$ & 2.05 \\
\hline 2 & $\$ 35.38$ & 2.17 & $\$ 35.92$ & 2.15 & $\$ 36.96$ & 2.10 \\
\hline 3 & $\$ 35.36$ & 2.18 & $\$ 35.99$ & 2.16 & $\$ 37.08$ & 2.07 \\
\hline 4 & $\$ 35.43$ & 2.19 & $\$ 35.89$ & 2.17 & $\$ 37.09$ & 2.08 \\
\hline 5 & $\$ 35.51$ & 2.21 & $\$ 35.80$ & 2.14 & $\$ 37.01$ & 2.08 \\
\hline AVERAGE & $\$ 35.39$ & 2.18 & $\$ 35.91$ & 2.15 & $\$ 37.05$ & 2.08 \\
\hline
\end{tabular}

Table 30: Results for day-and-night charging under TOU (50 users)

\begin{tabular}{|c|c|c|c|c|c|c|}
\hline \multirow{3}{*}{ Instance } & \multicolumn{5}{|c|}{ Penetration rate of medium-distance users } \\
\cline { 2 - 7 } & \multicolumn{2}{|c|}{$\mathbf{2 0 \%}$} & \multicolumn{2}{c|}{$\mathbf{3 5 \%}$} & \multicolumn{2}{c|}{$\mathbf{5 0 \%}$} \\
\cline { 2 - 7 } & $\begin{array}{c}\text { Total } \\
\text { Electricity } \\
\text { Cost }\end{array}$ & PAR & $\begin{array}{c}\text { Total } \\
\text { Electricity } \\
\text { Cost }\end{array}$ & PAR & $\begin{array}{c}\text { Total } \\
\text { Electricity } \\
\text { Cost }\end{array}$ & PAR \\
\hline 1 & $\$ 175.14$ & 2.18 & $\$ 179.90$ & 2.13 & $\$ 184.91$ & 2.09 \\
\hline 2 & $\$ 175.84$ & 2.19 & $\$ 179.54$ & 2.13 & $\$ 184.08$ & 2.07 \\
\hline 3 & $\$ 175.43$ & 2.18 & $\$ 179.66$ & 2.13 & $\$ 184.77$ & 2.08 \\
\hline 4 & $\$ 175.63$ & 2.17 & $\$ 179.73$ & 2.13 & $\$ 184.39$ & 2.08 \\
\hline 5 & $\$ 175.66$ & 2.19 & $\$ 179.35$ & 2.14 & $\$ 184.85$ & 2.06 \\
\hline AVERAGE & $\$ 175.54$ & 2.18 & $\$ 179.64$ & 2.13 & $\$ 184.60$ & 2.08 \\
\hline
\end{tabular}


Table 31: Results for day-and-night charging under TOU (100 users)

\begin{tabular}{|c|c|c|c|c|c|c|}
\hline \multirow{3}{*}{ Instance } & \multicolumn{3}{|c|}{ Penetration rate of medium-distance users } \\
\cline { 2 - 7 } & \multicolumn{2}{|c|}{$\mathbf{2 0 \%}$} & \multicolumn{2}{c|}{$\mathbf{3 5 \%}$} & \multicolumn{2}{c|}{$\mathbf{5 0 \%}$} \\
\cline { 2 - 7 } & $\begin{array}{c}\text { Total } \\
\text { Electricity } \\
\text { Cost }\end{array}$ & PAR & $\begin{array}{c}\text { Total } \\
\text { Electricity } \\
\text { Cost }\end{array}$ & PAR & $\begin{array}{c}\text { Total } \\
\text { Electricity } \\
\text { Cost }\end{array}$ & PAR \\
\hline 1 & $\$ 350.85$ & 2.17 & $\$ 359.26$ & 2.12 & $\$ 368.84$ & 2.07 \\
\hline 2 & $\$ 350.59$ & 2.17 & $\$ 359.96$ & 2.13 & $\$ 368.31$ & 2.06 \\
\hline 3 & $\$ 350.80$ & 2.18 & $\$ 359.23$ & 2.13 & $\$ 368.33$ & 2.08 \\
\hline 4 & $\$ 350.74$ & 2.19 & $\$ 359.64$ & 2.12 & $\$ 368.03$ & 2.07 \\
\hline 5 & $\$ 350.57$ & 2.17 & $\$ 360.03$ & 2.12 & $\$ 368.50$ & 2.07 \\
\hline AVERAGE & $\$ 350.71$ & 2.18 & $\$ 359.62$ & 2.13 & $\$ 368.40$ & 2.07 \\
\hline
\end{tabular}

Table 32: Results for day-and-night charging under TOU (200 users)

\begin{tabular}{|c|c|c|c|c|c|c|}
\hline \multirow{2}{*}{ Instance } & \multicolumn{6}{|c|}{ Penetration rate of medium-distance users } \\
\cline { 2 - 7 } & \multicolumn{2}{|c|}{$\mathbf{2 0 \%}$} & \multicolumn{2}{c|}{$\mathbf{3 5 \%}$} & \multicolumn{2}{c|}{$\mathbf{5 0 \%}$} \\
\cline { 2 - 7 } & $\begin{array}{c}\text { Total } \\
\text { Electricity } \\
\text { Cost }\end{array}$ & PAR & $\begin{array}{c}\text { Total } \\
\text { Electricity } \\
\text { Cost }\end{array}$ & PAR & $\begin{array}{c}\text { Total } \\
\text { Electricity } \\
\text { Cost }\end{array}$ & PAR \\
\hline 1 & $\$ 701.32$ & 2.17 & $\$ 719.57$ & 2.13 & $\$ 736.78$ & 2.08 \\
\hline 2 & $\$ 701.67$ & 2.18 & $\$ 719.39$ & 2.12 & $\$ 737.18$ & 2.07 \\
\hline 3 & $\$ 702.02$ & 2.18 & $\$ 719.95$ & 2.12 & $\$ 737.46$ & 2.07 \\
\hline 4 & $\$ 701.61$ & 2.18 & $\$ 719.21$ & 2.12 & $\$ 737.22$ & 2.07 \\
\hline 5 & $\$ 702.14$ & 2.18 & $\$ 719.68$ & 2.13 & $\$ 736.67$ & 2.08 \\
\hline AVERAGE & $\$ 701.75$ & 2.18 & $\$ 719.56$ & 2.13 & $\$ 737.06$ & 2.07 \\
\hline
\end{tabular}

Table 33: Optimal day-and-night charging versus uncontrolled charging under TOU

\begin{tabular}{|c|c|c|c|c|c|c|c|c|}
\hline \multirow{2}{*}{$\begin{array}{c}\text { Penetration } \\
\text { rate of } \\
\text { medium- } \\
\text { distance } \\
\text { users }\end{array}$} & \multicolumn{2}{|c|}{$\mathrm{n=10}$} & \multicolumn{2}{c|}{$\mathrm{n=50}$} & \multicolumn{2}{c|}{$\mathbf{n = 1 0 0}$} & \multicolumn{2}{c|}{$\mathrm{n=200}$} \\
\cline { 2 - 9 } & $\begin{array}{c}\text { Cost } \\
\text { reduced } \\
(\%)\end{array}$ & $\begin{array}{c}\text { PAR } \\
\text { reduced } \\
(\%)\end{array}$ & $\begin{array}{c}\text { Cost } \\
\text { reduced } \\
(\%)\end{array}$ & $\begin{array}{c}\text { PAR } \\
\text { reduced } \\
(\%)\end{array}$ & $\begin{array}{c}\text { Cost } \\
\text { reduced } \\
(\%)\end{array}$ & $\begin{array}{c}\text { PAR } \\
\text { reduced } \\
(\%)\end{array}$ & $\begin{array}{c}\text { Cost } \\
\text { reduced } \\
(\%)\end{array}$ & $\begin{array}{c}\text { PAR } \\
\text { reduced } \\
(\%)\end{array}$ \\
\hline $20 \%$ & 9.23 & 6.00 & 9.37 & 5.93 & 9.40 & 5.85 & 9.34 & 6.13 \\
\hline $35 \%$ & 8.90 & 5.28 & 8.73 & 5.93 & 8.65 & 6.01 & 8.64 & 5.89 \\
\hline $50 \%$ & 8.12 & 6.28 & 7.76 & 5.89 & 7.94 & 6.03 & 8.00 & 6.16 \\
\hline
\end{tabular}


Table 33 displays the total cost and PAR reduction for 10, 50, 100 and 200 users under the optimal day-and-night charging scenario compared to the uncontrolled EV charging. The results in Table 33 are similar to the results in Table 28. We note that there is no significant relationship between number of users $n$ and the improvements in total cost and PAR under the time-of-use pricing structure for the day-and-night charging model. Figure 12 depicts the results of the optimal day-and-night charging model for 10 users. From Figure 12, the energy demand between $6: 00 \mathrm{pm}$ to midnight is shifted entirely to between midnight and 7:00 am. However, we see some peaks between 8:00 am $(t=16)$ and 10:00 am $(t=20)$ due to enforced EV charging for medium-distance users.

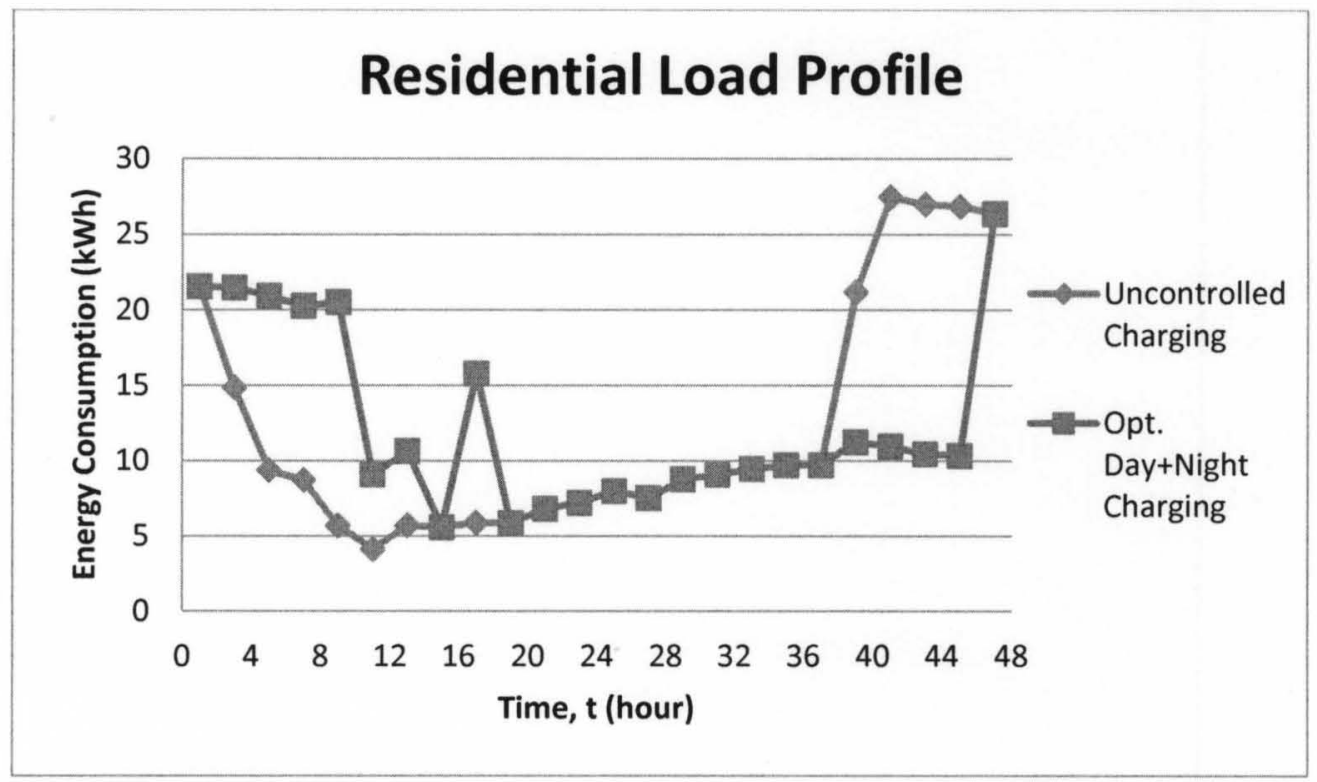

Figure 12: Load leveling under optimal day-and-night charging (TOU) 


\subsubsection{Comparisons between results for night-only and day-and-night charging}

Figure 13 shows a comparison between the charging scheduling for a mediumdistance User 1 who requires 9 total hours of charging for EV demand of $36 \mathrm{kWh}$. From Figure 13, we notice that the intervals for optimal scheduling overlaps for both charging scenarios. However, since we require all medium-distance users to charge at least for 1 hour during the day, User 1 is scheduled to charge his/her EV once at 8:00 am. We note that all the results are similar for day-and-night charging model where the mediumdistance users are only scheduled to charge once during the day instead of multiple times in the optimization model under a linear cost function. In addition, none of the shortdistance users are scheduled to charge under the day for the day-and-night charging scenario using time-of-use pricing structure.

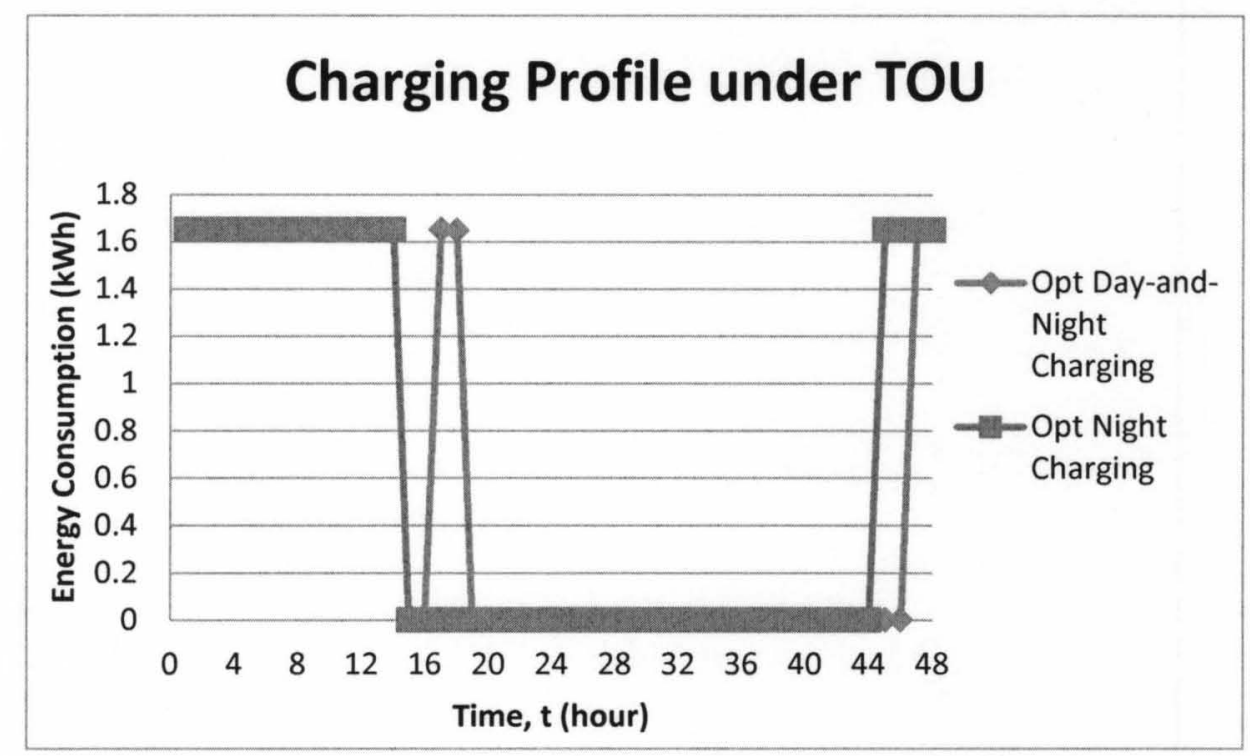

Figure 13: Charging profile for User 1 under time-of-use pricing structure 
We also observe that in all our numerical results for under the time-of-use cost, the day-and-night charging models incur a slightly higher cost compared to the night-only charging models. This observation is mainly due to the scheduled charging of mediumdistance EV users between 8:00 am to 5:30 pm which coincides with the peak hours. Hence, the highest price of 8 cents is incurred to the medium-distance users who charge during the peak hours.

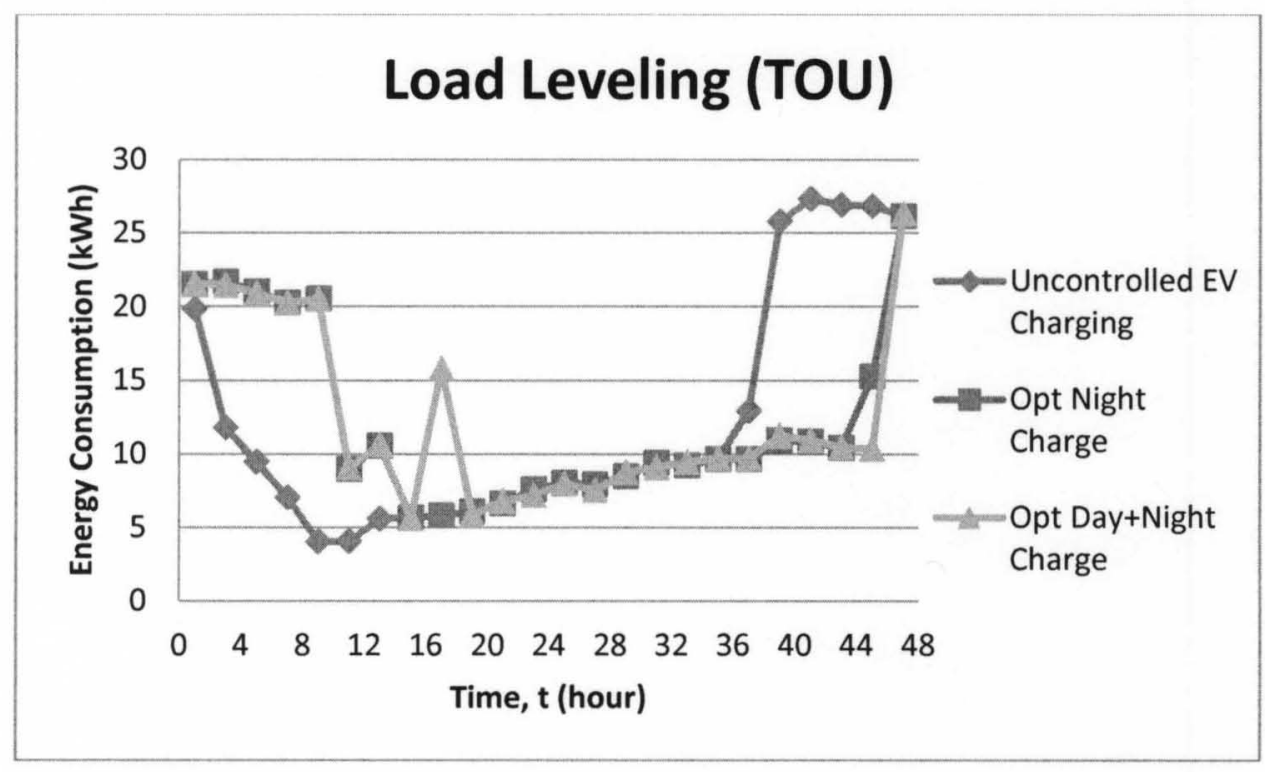

Figure 14: Overall load leveling under TOU pricing structure

Figure 14 displays the overall load leveling achieved for two charging scenarios under the time-of-use pricing structure. From Figure 14, we observe that the charging schedules for both scenarios are very alike except for a noticeable peak during the day under the day-and-night charging scenario. This is due to a constraint that enforces all medium-distance users to charge during the day $(8: 00 \mathrm{am}-5: 30 \mathrm{pm})$. Apart from that, there is not much difference between the charging profiles under the two charging scenarios. Thus, we conclude that time-of-use pricing structure is very effective in 
leveling the load and should be considered by the utility companies. However, we note that the pricing structure proposed in Table 2 by Collins and Mader (1983) is not very relevant since they proposed a much longer on-peak duration which starts from 7:00 am to $10: 00 \mathrm{pm}$. Hence, this result implies that detailed investigations have to be done such that a more relevant pricing structure can be implemented to users.

Furthermore, we note that increasing the number of users will not cause any significant effects on the total electricity cost incurred and PAR. The is mainly because that the time-of-use pricing structure itself has some optimization capability with respect to load leveling, which makes the effect of the optimization models less pronounced. Finally, when compared to the linear cost structure, the time-of-use cost structure induces more efficient load profiles without any optimization functions. The only drawback is that the time-of-use inevitably creates a shifted load peak at the time when the price starts to drop.

\subsection{Results for decentralized optimal charging model}

In this section, we present the numerical results for the decentralized day-andnight charging scheduling scenario as discussed in Chapter 4. Tables 34, 35, 36 and 37 display the total cost of electricity usage for $10,50,100$ and $200 \mathrm{EV}$ users, respectively. In Table 34, the total cost for 10 users at $20 \%$ penetration rate show that the "nearoptimal" charging schedule is $\$ 209.24$. The total cost increases slightly to $\$ 215.52$ at $35 \%$ penetration rate and further increases to $\$ 225.64$ at $50 \%$ penetration rate of medium-

distance users. Similar observations are seen for 50, 100 and 200 users. In general, a 
"near-optimal" charging schedule is achieved at day 3 for all users regardless of the penetration rate of medium-distance users.

Table 34: Results for decentralized model (10 users)

\begin{tabular}{|c|c|c|c|}
\hline \multirow{2}{*}{ Day } & \multicolumn{3}{|c|}{ Penetration of medium-distance users } \\
\cline { 2 - 4 } & $\mathbf{2 0 \%}$ & $\mathbf{3 5 \%}$ & $\mathbf{5 0 \%}$ \\
\hline 1 & $\$ 207.87$ & $\$ 212.89$ & $\$ 225.64$ \\
\hline 2 & $\$ 261.16$ & $\$ 275.12$ & $\$ 294.96$ \\
\hline 3 & $\$ 209.24$ & $\$ 215.52$ & $\$ 225.64$ \\
\hline 4 & $\begin{array}{c}\text { No further } \\
\text { improvement }\end{array}$ & $\begin{array}{c}\text { No further } \\
\text { improvement }\end{array}$ & $\begin{array}{c}\text { No further } \\
\text { improvement }\end{array}$ \\
\hline
\end{tabular}

Table 35: Results for decentralized model (50 users)

\begin{tabular}{|c|c|c|c|}
\hline \multirow{2}{*}{ Day } & \multicolumn{3}{|c|}{ Penetration of medium-distance users } \\
\cline { 2 - 4 } & $\mathbf{2 0 \%}$ & $\mathbf{3 5 \%}$ & $\mathbf{5 0 \%}$ \\
\hline 1 & $\$ 4,340.87$ & $\$ 4,507.18$ & $\$ 4,755.25$ \\
\hline 2 & $\$ 5,668.38$ & $\$ 6,121.57$ & $\$ 6,494.47$ \\
\hline 3 & $\$ 4,372.04$ & $\$ 4,568.89$ & $\$ 4,755.25$ \\
\hline 4 & $\begin{array}{c}\text { No further } \\
\text { improvement }\end{array}$ & $\begin{array}{c}\text { No further } \\
\text { improvement }\end{array}$ & $\begin{array}{c}\text { No further } \\
\text { improvement }\end{array}$ \\
\hline
\end{tabular}

Table 36: Results for decentralized model (100 users)

\begin{tabular}{|c|c|c|c|}
\hline \multirow{2}{*}{ Day } & \multicolumn{3}{|c|}{ Penetration of medium-distance users } \\
\cline { 2 - 4 } & $\mathbf{2 0 \%}$ & $\mathbf{3 5 \%}$ & $\mathbf{5 0 \%}$ \\
\hline 1 & $\$ 16,943.78$ & $\$ 17,654.69$ & $\$ 18,554.99$ \\
\hline 2 & $\$ 22,234.95$ & $\$ 24,403.38$ & $\$ 25,478.68$ \\
\hline 3 & $\$ 17,070.62$ & $\$ 17,654.69$ & $\$ 18,554.99$ \\
\hline 4 & $\begin{array}{c}\text { No further } \\
\text { improvement }\end{array}$ & $\begin{array}{c}\text { No further } \\
\text { improvement }\end{array}$ & $\begin{array}{c}\text { No further } \\
\text { improvement }\end{array}$ \\
\hline
\end{tabular}


Table 37: Results for decentralized model (200 users)

\begin{tabular}{|c|c|c|c|}
\hline \multirow{2}{*}{ Day } & \multicolumn{3}{|c|}{ Penetration of medium-distance users } \\
\cline { 2 - 4 } & $\mathbf{2 0 \%}$ & $\mathbf{3 5 \%}$ & $\mathbf{5 0 \%}$ \\
\hline 1 & $\$ 66,985.22$ & $\$ 69,791.27$ & $\$ 73,396.75$ \\
\hline 2 & $\$ 88,218.39$ & $\$ 96,833.07$ & $\$ 101,104.02$ \\
\hline 3 & $\$ 67,486.05$ & $\$ 69,791.27$ & $\$ 73,396.75$ \\
\hline 4 & $\begin{array}{c}\text { No further } \\
\text { improvement }\end{array}$ & $\begin{array}{c}\text { No further } \\
\text { improvement }\end{array}$ & $\begin{array}{c}\text { No further } \\
\text { improvement }\end{array}$ \\
\hline
\end{tabular}

Table 38 summarizes the performance measures for various numbers of users with an increasing penetration rate of medium-distance users. From Table 38, the total cost increases as the penetration rate increases. The PAR, on the other hand, decreases with an increasing penetration rate of medium-distance users. However, a general observation shows that increasing the number of users from 10 to 200 has no significant impact on PAR. In particular, at a fixed penetration rate of $35 \%$, the PAR for $10,50,100$ and 200 users is calculated to be 1.80 .

Table 38: Summary of results for decentralized model

\begin{tabular}{|c|c|c|c|c|c|c|}
\hline \multirow{3}{*}{$\mathbf{n}$} & \multicolumn{6}{|c|}{ Penetration rate of medium-distance users } \\
\cline { 2 - 7 } & \multicolumn{2}{|c|}{$\mathbf{2 0 \%}$} & \multicolumn{2}{|c|}{$\mathbf{3 5 \%}$} & \multicolumn{2}{c|}{$\mathbf{5 0 \%}$} \\
\cline { 2 - 7 } & Total Cost & PAR & Total Cost & PAR & Total Cost & PAR \\
\hline 10 & $\$ 209.24$ & 1.83 & $\$ 215.52$ & 1.80 & $\$ 225.64$ & 1.83 \\
\hline 50 & $\$ 4,372.04$ & 1.84 & $\$ 4,568.89$ & 1.80 & $\$ 4,755.25$ & 1.77 \\
\hline 100 & $\$ 17,070.62$ & 1.84 & $\$ 17,654.69$ & 1.80 & $\$ 18,554.99$ & 1.77 \\
\hline 200 & $\$ 67,486.05$ & 1.84 & $\$ 69,791.27$ & 1.80 & $\$ 73,396.75$ & 1.77 \\
\hline
\end{tabular}


Table 39: Improvements of decentralized model

\begin{tabular}{|c|c|c|c|c|c|c|c|c|}
\hline \multirow{2}{*}{$\begin{array}{c}\text { Penetration } \\
\text { rate of } \\
\text { medium- } \\
\text { distance } \\
\text { users }\end{array}$} & \multicolumn{2}{|c|}{$n=10$} & \multicolumn{2}{|c|}{$\mathrm{n}=\mathbf{5 0}$} & \multicolumn{2}{|c|}{$\mathrm{n}=\mathbf{1 0 0}$} & \multicolumn{2}{|c|}{$\mathrm{n}=\mathbf{2 0 0}$} \\
\hline & $\begin{array}{c}\text { Cost } \\
\text { reduced } \\
(\%)\end{array}$ & $\begin{array}{c}\begin{array}{c}\text { PAR } \\
\text { reduced } \\
(\%)\end{array} \\
\end{array}$ & $\begin{array}{c}\begin{array}{c}\text { Cost } \\
\text { reduced } \\
(\%)\end{array} \\
\end{array}$ & $\begin{array}{c}\begin{array}{c}\text { PAR } \\
\text { reduced } \\
(\%)\end{array} \\
\end{array}$ & $\begin{array}{c}\begin{array}{c}\text { Cost } \\
\text { reduced } \\
(\%)\end{array} \\
\end{array}$ & $\begin{array}{c}\begin{array}{c}\text { PAR } \\
\text { reduced } \\
(\%)\end{array} \\
\end{array}$ & $\begin{array}{c}\begin{array}{c}\text { Cost } \\
\text { reduced } \\
(\%)\end{array} \\
\end{array}$ & $\begin{array}{c}\text { PAR } \\
\text { reduced } \\
(\%)\end{array}$ \\
\hline $20 \%$ & 13.23 & 21.46 & 14.56 & 20.66 & 14.74 & 20.37 & 14.83 & 20.52 \\
\hline $35 \%$ & 12.10 & 20.85 & 13.35 & 20.79 & 14.46 & 20.54 & 14.54 & 20.50 \\
\hline $50 \%$ & 12.08 & 17.55 & 12.86 & 19.94 & 13.06 & 19.67 & 13.32 & 19.74 \\
\hline
\end{tabular}

Table 39 presents the improvements of decentralized model compared to an uncontrolled charging scenario. The results from Table 39 show that the improvements in total cost increases as we increase the number of users. Hence, this result implies that the decentralized model becomes more efficient in cost reduction when a larger number of EV users are involved. For example, at $20 \%$ penetration rate, the total cost improves from $13.23 \%$ to $14.83 \%$ when the number of users increases from 10 to 200 users. However, in general, the improvements in total cost and PAR for each user size $n$ reduces as we increase the penetration rate from $20 \%$ to $50 \%$.

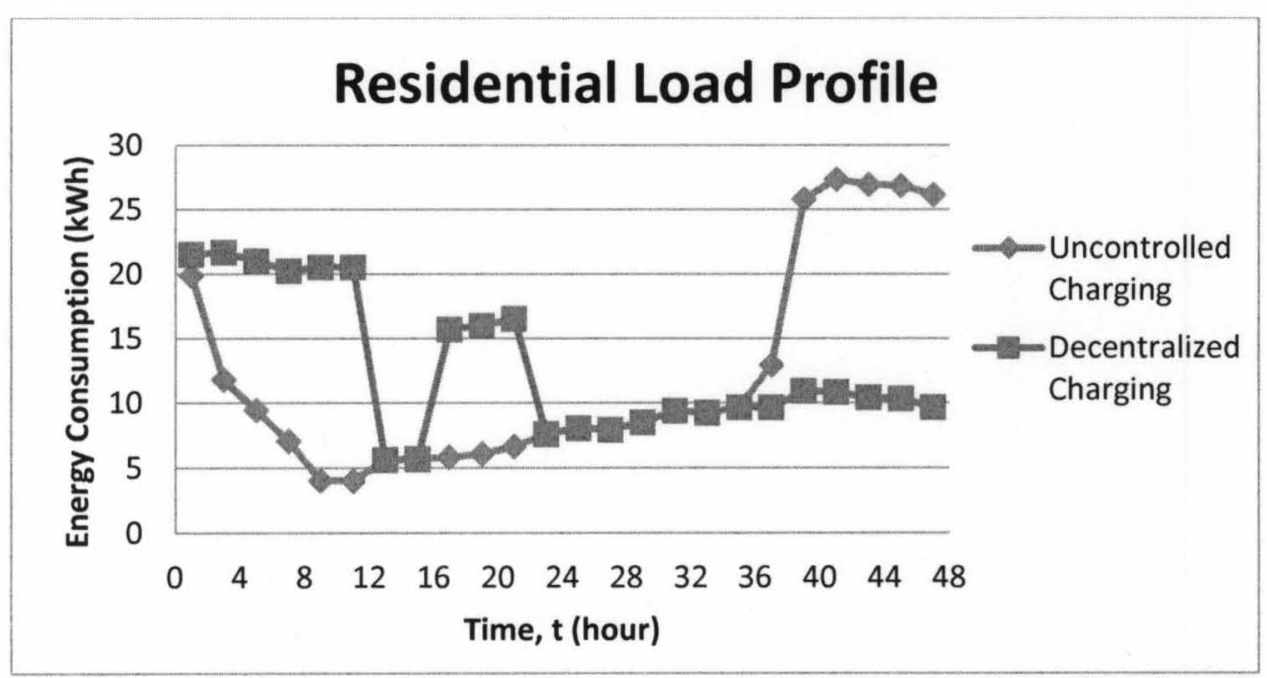

Figure 15: Residential load profile under decentralized model 
Figure 15 depicts a residential load profile for 10 users under two different charging scenarios. In Figure 15, the peak load due to uncontrolled charging between 6:00 pm to midnight is substantially reduced from approximately $27 \mathrm{kWh}$ to approximately $10 \mathrm{kWh}$ of load. We notice that the peak load under the decentralized model exists between midnight and 5:00 am. Slightly lower peaks in the decentralized load profile are also seen during the day due to daytime charging by some of the EV users. We observe comparable load profiles for 50, 100 and 200 users. From the load profiles, we can deduce that the users shift their night-charging schedule to the duration where the household demand is the lowest, i.e., between midnight to 5:00 am.

\subsection{Discussions on centralized versus decentralized models}

When we compare the results for the day-and-night charging scenario using two different approaches, we find that the centralized charging scheduling models are more efficient in improving the performance measures. The total cost reduces to approximately $19 \%$ when we employ the centralized model, while the decentralized model reduces the total cost to approximately $14 \%$. Correspondingly, the PAR reduction is approximately $41 \%$ when we utilize the centralized model compared to only $20 \%$ reduction under a decentralized model. Nonetheless, the results show that on the whole, both approaches of optimization models provide some improvements over the uncontrolled charging scenario.

Moreover, we note that since we are applying essentially coordinate search to a discrete optimization problem, the decentralized charging scheduling model does not 
guarantee global optimality. Hence, the decentralized model is considered a distributed heuristic method. We also notice some alternation in the results from the decentralized model. Due to this alternation, we terminate the heuristic after 6 consecutive days if no further improvement is made. Hence, there could be a possibility that early termination of the heuristic causes the results to not be truly optimal. 


\section{CHAPTER 6: CONCLUSIONS AND FUTURE WORK}

\subsection{Conclusions}

In this thesis, we study a charging scheduling problem for BEV users in residential areas to mitigate the impact of uncontrolled charging on the peak load during evening hours. We develop several optimization models based on two approaches which aim to minimize the total electricity cost for all users while meeting the user's household and EV charging demands.

In our first approach, we used a mixed integer non-linear programming in modeling the centralized optimal charging models for BEV users under different cost functions and charging scenarios. We draw several conclusions based on our numerical results. First, using linear cost as the objective function, we conclude that overall there is an approximately $19 \%$ and $27 \%$ in cost reduction for night-only charging scenario and day-and-night charging scenario, respectively. Furthermore, the overall reduction in PAR compared to the uncontrolled charging from BEV users is approximately $41 \%$ under the night-only charging model. However, a slightly higher improvement (53\%) can be achieved if we implement the day-and-night charging scenario.

Second, we conclude that there was not much improvement in total electricity cost and the PAR for two scenarios under the time-of-use pricing structure. This indicates 
that the time-of-use pricing structure itself is effective in achieving load leveling. Thus, it should be strongly considered by utility companies. Based on our numerical results, we suggest that the utility companies consider detailed investigations and considerations in establishing the hours and price for off-peak, intermediate and peak durations. Between the two cost structures, the optimization models under the linear cost function offer more significant reduction in total electricity cost for all users.

Third, as the number of BEV user increases, the optimal charging scheduling models result in an increased improvement for both total electricity cost and PAR compared to the uncontrolled charging scenario. Additionally, we conclude that increasing the penetration rate of medium-distance users would not significantly impact the improvement in the total electricity cost and the PAR made by optimal scheduling.

In our second approach, we establish a mixed integer linear programming in modeling a decentralized charging scheduling algorithm for BEV users. Under this decentralized approach, BEV users optimize their own charging schedule instead of following the scheduling proposed by central controller. In general, the decentralized model provides an approximately $14 \%$ improvement in total cost and $20 \%$ improvement in PAR compared to the uncontrolled charging scenario. We speculate that although the improvement shown by the decentralized model is not as significant as the results from our centralized models, the decentralized approach is perhaps more preferable to EV users.

In conclusion, all the optimization models that we propose in this thesis show some improvement, if not more, against the uncontrolled EV charging scenario by the 
users. Implementing the optimal charging scheduling model will significantly level the load profile as we envision higher penetration of EV users in the near future.

\subsection{Future Work}

There are several topics in our future research. First, we would like to develop a customized algorithm for solving large-size centralized models under both linear and time-of-use cost structures. Second, we would also like to improve the decentralized optimization model to achieve a better charging schedule that is closer to the optimal charging schedule from the centralized model.

Third, we plan on investigating the effect of implementing separate charging periods for different travel motions as proposed by Koyanagi and Uriu (1998). Particularly, we will set different allowable charging periods for medium-distance and short-distance BEV users so load leveling can be achieved to some extent by implementing such policies. Finally, we would also like to study the optimization models under a piecewise linear cost function. The piecewise linear function, widely adopted by power generation companies, represents an increasing unit cost that will be incurred to each user for an increasing amount of energy usage. For instance, a price of 4.42 cents is charged to a user for the first $25 \mathrm{kWh}$ of energy consumed daily, and a higher price of 5.50 cents will be charged for the next $15 \mathrm{kWh}$ of energy consumed in a day. The highest price of 7.50 cents will be charged for any energy consumed beyond $40 \mathrm{kWh}$ in a day. Similar pricing structure is adopted by utility companies in California. It would be interesting to investigate the impact of optimal scheduling for EV charging under such pricing structure. 


\section{REFERENCES}

Aigner, D. (1985). The residential electricity time-of-use pricing experiments: what have we learned? In Hausman, J. A. \& Wise, D. A. (Eds.), Social Experimentation, Chicago: University of Chicago Press, 11-53.

Baladi, S. M., Herriges, J. A. \& Sweeney, J. T. (1998). Residential response to voluntary time-of-use electricity rates. Resource and Energy Economics, 20, 225-244.

Bonabeau, E. (2002, May 14). Agent-based modeling: Methods and techniques for simulating human systems. Proceedings of the National Academy of Science, 99(3), 7280-7287.

Cain, M. B. \& Alvarado, F. L. (2004, July). Implications of cost and bid format on electricity market studies: linear versus quadratic costs. IEEE Large Engineering Systems Conference on Power Engineering, Halifax, Canada, 2-6.

Clement-Nyns, K., Haesen, E. \& Driesen, J. (2010, February). The impact of charging plug-in hybrid electric vehicles on a residential distribution grid. IEEE Transactions on Power Systems, 25(1), 371-380.

Collins, M. \& Mader, G. (1983, February). The timing of EV recharging and its effect on utilities. IEEE Transactions on Vehicular Technology, 32(1), 90-97.

CPLEX: CPLEX Optimization Inc. (1996). Incline Village.

DeCicco, J., Fung, F. \& An, F. (2006). Global warming on the road: The Climate Impact of America's Automobiles. Retrieved October 20, 2011, from http://www.edf.org/sites/default/files/5301_Globalwarmingontheroad_0.pdf.

Deilami, S., Masoum, A. S., Moses, P. S. \& Masoum, M. A. S. (2011, September). Realtime coordination of plug-in electric vehicle charging in smart grids to minimize the power losses and improve voltage profile. IEEE Transactions on Smart Grid, 2(3), 456467.

Energy Information Administration. (2011). International Energy Outlook 2011. 
Fan, Z. (2010). Distributed demand response and user adaptation in smart grids. Simulation, 1-6.

Fetz, A. \& Filippini, M. (2010). Economies of vertical integration in the Swiss electricity sector. Energy Economics, 32(6), 1325-1330.

Galus, M. D. \& Andersson, G. (2008, November 17-18). Demand management of grid connected plug-in hybrid electric vehicles (PHEV). Proceedings IEEE Energy 2030 Conference, Atlanta, Georgia, 1-8.

GAMS: General Algebraic Modeling System. (1995). GAMS Development Corporation.

Gellings, C. W. (1985, October). The concept of demand-side management for electric utilities. Proceedings of the IEEE, 73(10), 1468-1470.

Hartway, R., Price, S. \& Woo, C. K. (1999). Smart meter, customer choice and profitable time-of-use rate option. Energy, 24(10), 859-903.

Ipakchi, A. \& Albuyeh, F. (2009, March-April). Grid of the future. IEEE Power and Energy Magazine, 7(2), 52-62.

Kowahl, N. \& Kuh, A. (2010, July 18-23). Micro-scale smart grid optimization. The 2010 International Joint Conference on Neural Networks (IJCNN), Barcelona, 1-8.

Koyanagi, F. \& Uriu, Y. (1998, August). A strategy of load leveling by charging and discharging time control of electric vehicles. IEEE Transactions on Power Systems, 13(3), 1179-1184.

Ma, Z., Callaway, D. \& Hiskens, I. (2010, December 15-17). Decentralized charging control for large populations of plug-in electric vehicles. $49^{\text {th }}$ IEEE Conference on Decision and Control, Atlanta, Georgia, 206-212.

Martinez-Budria, E., Jara-Diaz, S. \& Ramos Real, F. J. (2003). Adapting productivity theory to the quadratic cost function: an application to the Spanish electric sector. Journal of Productivity Analysis, 20(2), 213-229.

Mohsenian-Rad, A-H., Wong, V.W.S., Jatskevich, J. \& Schober, R. (2010, January 1921). Optimal and autonomous incentive-based energy consumption scheduling algorithm for smart grid. Innovative Smart Grid Technologies (ISGT), 1-6. 
NAHB Research Center, Inc. (2001, July 16). Review of residential electrical energy use data. Retrieved August 15, 2011, from http://www.toolbase.org/PDF/CaseStudies/ResElectricalEnergyUseData.pdf.

Nissan LEAF. (n.d.). Nissan LEAF: features and specifications. Nissan USA. Retrieved November 1, 2011, from http://www.nissanusa.com/leaf-electric-car/specsfeatures/index\#/leaf-electric-car/.

NV Energy. (n.d.). Residential time of use for southern service territory. Retrieved November 4, 2011 from http://nvenergy.com/home/paymentbilling/timeofuse.cfm.

Obara, S. (2007). Equipment arrangement planning of a fuel cell energy network optimized for cost minimization. Renewable Energy, 32(3), 382-406.

Portland General Electric. (n.d.). Residential pricing. Retrieved November 4, 2011 from $\mathrm{http}: / / \mathrm{www}$.portlandgeneral.com/residential/your_account/billing_payment/time_of_use/ pricing.aspx.

Rahman, S. \& Shrestha, G. B. (1993, April 2). An investigation into the impact of electric vehicle load on the electric utility distribution system. IEEE Transactions on Power Delivery, 8(2), 591-597.

Ramchurn, S., Vytelingum, P., Rogers, A. \& Jennings, N. (2011). Agent-based control for decentralized demand side management in the smart grid. The Tenth International Conference on Autonomous Agents and Multiagent Systems (AAMAS 2011), Taipei, Taiwan, 5-12.

Shafiee, S. \& Topal, E. (2009, January). When will fossil fuel reserves be diminished? Energy Policy, 37(1), 181-189.

Shahidehpour, M., Yamin, H. \& Li, Z. (2002). Market operations in electric power systems: forecasting, scheduling and risk management, New York: John Wiley and Sons Inc. 
Sortomme, E., Hindi, M. M., MacPherson, S. D. J. \& Venkata, S. S. (2011, March). Coordinated charging of plug-in hybrid electric vehicles to minimize distribution system losses. IEEE Transactions on Smart Grid, 2(1), 198-205.

Southern California Edison. (n.d.). Residential rates. Retrieved November 4, 2011 from http://www.sce.com/residential/rates/special-time-of-use.htm.

The Energy Foundation. (2006). Annual Report. Retrieved October 20, 2011, from http://www.ef.org/documents/2006_Annual_Report.pdf.

U.S. Department of Energy. (n.d.). All-electric vehicle basics. Alternative Fuels and Advanced Vehicles Data Center. Retrieved August 26, 2011, from http://www.afdc.energy.gov/afdc/vehicles/electric_basics_ev.html.

Vandael, S., Boucke, N., Holvoet, T., \& Deconinck, G. (2010). Decentralized demand side management of plug-in hybrid vehicles in a smart grid. Proceedings of First International Workshop on Agent Technologies for Energy Systems, 67-74.

Zhang, D., Papageorgiou, L. G., Samsatli, N. J. \& Shah, N. (2011, May 22-27). Optimal scheduling of smart homes energy consumption with microgrid. The First International Conference on Smart Grids, Green Communications and IT Energy-aware Technologies, Venice, Italy, 70-75.

Vytelingum, P., Voice, T. D., Ramchurn, S. D., Rogers, A. \& Jennings, N. R. (2010, May 10-14). Agent-based micro-storage management for the smart grid. The Ninth International Conference on Autonomous Agents and Multiagent Systems (AAMAS 2010), Toronto, Canada, 39-46. 


\section{CURRICULUM VITAE}

Name:

Address:

Education:

Awards:

Activities \& Honors:
Nur Dayana Abd Rahman

Department of Industrial Engineering J.B. Speed School of Engineering

University of Louisville

Louisville, KY 40292

B.E., Electrical Engineering Minor: Engineering Management

Vanderbilt University, Nashville, TN

2005-2009

M.S., Industrial Engineering

University of Louisville, Louisville, $\mathrm{KY}$

2010-2011

Walter Criley Award 2009

Vanderbilt University, Nashville, TN

Institute of Industrial Engineers

Golden Key International Honor Society

Tau Beta Pi (Engineering Honor Society)

Alpha Pi Mu (Industrial Engineering Honor Society)

Eta Kappa Nu (Electrical Engineering Honor Society) 